\title{
Tracking the Source of Enantioselectivity
}

\author{
by \\ Nina Leeb
}

A thesis

submitted to the Victoria University of Wellington in partial fulfilment of the requirements for the degree of Masters

Victoria University of Wellington

2015 


\section{Abstract}

Enantioselectivity remains one of synthetic chemistry's most formidable problems. It arises due to the formation of diastereomeric species in a reaction, either in the form of diastereomeric intermediates or a set of diastereomeric transition states. Without control a racemic mixture is formed. A resolution method is then required to separate the enantiomers. Any given resolution method will rely on the differing energies of diastereomers to allow for their separation. Experimentally there are a myriad of different options that may be used to induce separation; for example chromatophraphy and/or crystallisation. The actual process that occurs throughout this separation has not, however, been fully investigated in all cases. A better understanding of the process is able to provide an understanding of how resolution methods work i.e. when diastereomers occur and how great their energy differences are. This is vital in increasing the efficiency and effectiveness of any given resolution method. This theoretical study completed an investigation of the reaction pathway between the enantiomers of 2-formyl-3-hydroxyl[2.2]paracyclophane (FHPC) with (S)-valyl-(S)-valine. A subsequent investigation of an alternative resolution method, involving (R)- $\alpha$-PEAM, was also conducted. This latter resolution method was proposed experimentally as a simpler method that could aid in improving the separation of the enantiomers. This investigation was carried out using Density Functional Theory (DFT) with the PBE0 functional and the triple$\zeta$ TZVP basis set. The complete reaction profile was determined and diastereomeric intermediates and transition states for both resolution methods along two different pathways were determined; the 'N-deprotonation Pathway' and the 'Odeprotonation Pathway'. The inadequacy of the first resolution method was found to be due to the presence of copper(II). Furthermore it was discovered that the reaction for both pathways would most likely proceed through the 'O-deprotonation Pathway' due to the barriers being lower in energy. 


\section{Acknowledgements}

It is hard to believe that two years have gone by since I started this project. Those two years have been interesting and enjoyable and have sparked my interested in continuing on. Experimental chemistry was never something I was interested in. I loved chemistry for its theories and crazy ways. When I first learnt about Computational Chemistry in my second year at Victoria University I knew I had found the type of chemistry for me. Carrying out research was not something I was used to and using a computer as much as I was meant to terrified me. Now I like to think that I terrify people because of what I am able to do. I would not have been able to complete this research if it weren't for my supervisor, Dr. Matthias Lein. I know he had to put up with a lot of questions from me especially when it came to error messages. So thank you for your patience and your guidance.

I'd also like to thank the members of the group for putting up with me; Wong, Ivan and Kevin. I had no understand of computers when I started and I have learnt a lot from all of you. I'd like to especially thank Wong for dealing with my constant questions and reminding me that it is possible to finish a thesis without completely losing it.

I want to thank Dr. Joanne Harvey for taking the time to meet with me, answer my questions, and look over my mechanisms over and over again.

Thank you to my friends for understanding that chemistry can be hard and that sometimes you don't have time for anything else. I'd especially like to thank my friends who dealt with, what I like to call, 'Thesis Brain'. It could not have been easy but I hope you got a few laughs out of it.

Lastly I'd like to thank my family. Thank you Oli for letting me stay with you for two weeks so that I could get away from everything. And thank you to my parents. I would not be here if it weren't for you. I appreciate the fact that you let me stay here so much, even though I know you'd like me to be closer to home. 


\section{Contents}

Abstract

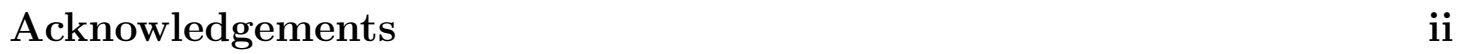

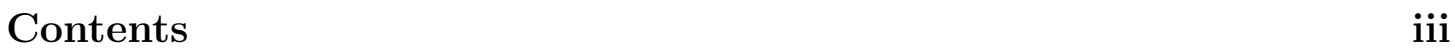

List of Figures $\quad$ v

List of Tables vii

List of Schemes viii

1 Introduction $\quad 1$

1.1 Enantioselectivity . . . . . . . . . . . . . . . 1

1.1.1 Diastereomers . . . . . . . . . . . . . . . 3

1.2 Asymmetric Synthesis . . . . . . . . . . . . . . . . . . 4

1.2.1 Chiral non-racemic solvent . . . . . . . . . . . . 6

1.2.2 Chiral non-racemic reagent . . . . . . . . . . . . . 7

1.2.3 Chiral non-racemic catalyst . . . . . . . . . . . . . 8

1.2.4 Chiral non-racemic substrate . . . . . . . . . . . . . . . . . 9

1.3 Cyclophanes .......................... 11

1.3.1 Ligands . . . . . . . . . . . . . . . . . . . . . . 18

1.4 Resolution Methods . . . . . . . . . . . . . . . . . . . . 27

1.5 Computational Chemistry . . . . . . . . . . . . . . 32

1.6 Research Aims . . . . . . . . . . . . . . . . . . 33

2 Methodology $\mathbf{3 5}$

2.1 Computational Methods . . . . . . . . . . . . . . . . 35

3 Results and Discussion (Resolution Method 1) 37

3.1 N-deprotonation Reaction Overview . . . . . . . . . . . . . . . 38

3.1.1 Transition State 1 . . . . . . . . . . . . . . 40

3.1.1.1 FHPC Rotational Barrier . . . . . . . . . . 40

3.1 .2 Transition State 2 . . . . . . . . . . . . . . . . . 43

3.1.2.1 Rotational Barrier . . . . . . . . . . 46 
3.2 O-deprotonation Reaction Overview . . . . . . . . . . . . . . 48

3.2.1 Transition State 2 . . . . . . . . . . . . . . . . 50

3.2.1.1 Rotational Barrier .............. 51

3.2 .2 Transition State $3 \ldots \ldots$. . . . . . . . . . . . . 53

3.3 Copper Coordinating Pathway . . . . . . . . . . . . . . . . 55

3.4 Thermodynamics . . . . . . . . . . . . . . 56

3.5 Solvent Effects . . . . . . . . . . . . . . . . . . 56

3.6 Discussion . . . . . . . . . . . . . . . . . . 57

3.6.1 N-deprotonation Pathway . . . . . . . . . . . . 57

3.6.2 O-deprotonation Pathway ............... 61

3.6 .3 Comparison . . . . . . . . . . . . . . . 63

3.6.4 Copper Coordinating Pathway . . . . . . . . . . . . 65

3.6.5 Thermodynamics . . . . . . . . . . . . . . . . 65

3.6.6 Solvent Effects . . . . . . . . . . . . . . . . 66

4 Results and Discussion (Resolution 2) $\quad 67$

4.1 N-deprotonation Pathway Overview . . . . . . . . . . . . . . . 68

4.1 Transition State 1 . . . . . . . . . . . . . . 70

4.1.1.1 Rotational Barrier . . . . . . . . . . 70

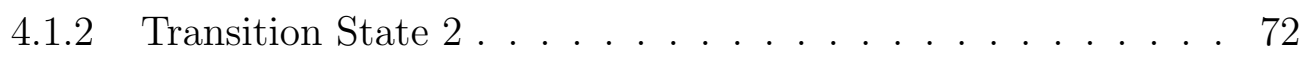

4.2 O-deprotonation Pathway Overview . . . . . . . . . . . . . . . 73

4.2 .1 Transition State 2 . . . . . . . . . . . . 76

4.2.1.1 Rotational Barrier . . . . . . . . . . . . 76

4.2 .2 Transition State $3 \ldots \ldots$. . . . . . . . . . . 78

4.3 Thermodynamics . . . . . . . . . . . . . . . . . . 79

4.4 Discussion . . . . . . . . . . . . . . . . . . . . 80

4.4.1 N-deprotonation Pathway . . . . . . . . . . . . . . 80

4.4 .2 O-deprotonation Pathway . . . . . . . . . . . . . 84

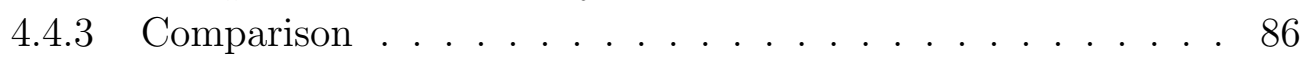

4.4.4 Thermodynamics . . . . . . . . . . . . . . . . 88

5 Comparison of Resolution Reactions $\quad 89$

5.1 N-Deprotonation Pathway . . . . . . . . . . . . . . . . . 89

5.2 O-Deprotonation Pathway . . . . . . . . . . . . . . . . 92

6 Conclusions $\quad 95$

$\begin{array}{lr}\text { Bibliography } & 97\end{array}$ 


\section{List of Figures}

1.1 Structure of thalidomide . . . . . . . . . . . . . . . 2

1.2 Structure of DOPA . . . . . . . . . . . . . . . . . . . . . . . . . . . . . . . . . .

1.3 Examples of enantiotopic faces . . . . . . . . . . . . . 5

1.4 Example of an enantiotopic group . . . . . . . . . . . . . . 5

1.5 General method for using a chiral auxiliary . . . . . . . . . . . . . . 10

1.6 Two examples of paracyclophanes . . . . . . . . . . . . . . . . . 12

$1.7[2.2]$ paracyclophane structure . . . . . . . . . . . . . . . . . . . . . . . . . . . 12

1.8 Assigning planar chirality . . . . . . . . . . . . . . . . . . . . . . . . . . . . . . . . . 13

1.9 Structure of FHPC . . . . . . . . . . . . . . . . . . . . 16

1.10 Structure of PHANEPHOS . . . . . . . . . . . . . . . 18

1.11 Diastereomeric ligands for $\beta$-boration . . . . . . . . . . . . . . . 19

1.12 Two ligands used in a copper-catalysed Henry reaction . . . . . . . 21

1.13 Structure of Br-AHPC and Br-BHPC . . . . . . . . . . . . . . . 21

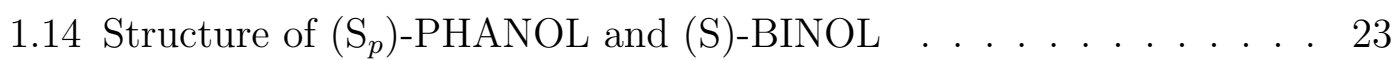

1.15 Diastereomic ligands for addition of diethylzinc . . . . . . . . . . . 24

1.16 Ligands for addition of diethylzinc to benzaldehyde . . . . . . . . . 25

1.17 Structure of iso-FHPhPC and pseudo-FHPhPC . . . . . . . . . . 27

1.18 Structure of both enantiomers of 4-formyl[2.2]paracyclophane . . . . 29

1.19 Chiral auxiliary used for the thio-Claisen rearrangement . . . . . . 32

3.1 The reaction pathway for the 'N-deprotonation Pathway' of the first resolution method is shown . . . . . . . . . . . . . 39

3.2 TS1 for the "N-deprotonation Pathway' . . . . . . . . . . . . . . . 41

3.3 Cis-like conformers, trans-like conformer, and TS for FHPC . . . . 42

3.4 Plot of the rotational barrier for FHPC . . . . . . . . . . . . . . 43

3.5 TS2 for the 'N-deprotonation Pathway' . . . . . . . . . . . . . . . . 44

3.6 Diastereomeric copper complexes . . . . . . . . . . . . . . . 45

3.7 Rotational Barrier Plot for $\left(\mathrm{S}_{p}\right)$-Min3 . . . . . . . . . . . . . 47

3.8 Cis-like conformer, trans-like conformer, and TS for the ' $\mathrm{N}$-deprotonation Pathway' of the first resolution method . . . . . . . . . . . . . . . 48

3.9 The reaction pathway for the 'O-deprotonation Pathway' of the first resolution method is shown . . . . . . . . . . . . . . 49

3.10 TS2 for the 'O-deprotonation Pathway' . . . . . . . . . . . . . . . 52

3.11 TS3 for the 'O-deprotonation Pathway' . . . . . . . . . . . . . 54

4.1 Structure of two amines . . . . . . . . . . . . . . . . 67 
4.2 The reaction pathway for the 'N-deprotonation Pathway' of the second resolution method is shown . . . . . . . . . . . . 69

4.3 TS1 for the 'N-deprotonation Pathway' . . . . . . . . . . . . . . . . 71

4.4 TS2 for the 'N-deprotonation Pathway' . . . . . . . . . . . . . . . . 74

4.5 The reaction pathway for the 'O-deprotonation Pathway' of the second resolution method is shown . . . . . . . . . . . . 75

4.6 TS2 for the 'O-deprotonation Pathway' . . . . . . . . . . . . . . 77

4.7 TS3 for the 'O-deprotonation Pathway' . . . . . . . . . . . . . . 79 


\section{List of Tables}

3.1 FHPC rotational barrier . . . . . . . . . . . . . . . . 43

3.2 Rotational Barrier for the 'N-deprotonation Pathway' for the first resolution method . . . . . . . . . . . . . . . . 46

3.3 Rotational barrier for the 'O-deprotonation Pathway' for the first resolution method . . . . . . . . . . . . . . . . 53

3.4 Copper reaction . . . . . . . . . . . . . . . . . . . 55

$3.5 \Delta \mathrm{G}$ for the overall reaction . . . . . . . . . . . . . . 56

3.6 Copper solvent effects . . . . . . . . . . . . . . 57

4.1 Rotational Barrier for the 'N-deprotonation Pathway' of the second resolution method . . . . . . . . . . . . . . . . . 72

4.2 Rotational Barrier for the 'O-deprotonation Pathway' of the second resolution method . . . . . . . . . . . . . . 78

$4.3 \Delta \mathrm{G}$ for the overall reaction . . . . . . . . . . . . . 80 


\section{List of Schemes}

1.1 Use of a chiral boronate in asymmetric synthesis . . . . . . . . . . . 7

1.2 Asymmetric hydrogenation reaction . . . . . . . . . . . . . . . . 9

1.3 Use of an Evans auxiliary . . . . . . . . . . . . . . . . . 11

1.4 Introducing chirality to $[2.2]$ paracyclophane . . . . . . . . . . . 14

1.5 Synthesis of FHPC . . . . . . . . . . . . . . . . . . . . 15

1.6 Monosubstituted [2.2] paracyclophane derivative for addition of isopropylzinc . . . . . . . . . . . . . . . . 16

1.7 Synthesis of racemic FHPC . . . . . . . . . . . . . . . 17

1.8 Synthesis of $\beta$-hydroxy- $\alpha$-amino acids . . . . . . . . . . . . . . . . 18

1.9 Resolution of FHPC using (S)-valyl-(S)-valine . . . . . . . . . . . . 30

1.10 Resolution of FHPC using $(\mathrm{R})-\alpha-\mathrm{PEAM} \ldots$. . . . . . . . . . . . . . 31 


\section{Chapter 1}

\section{Introduction}

An important part in synthetic chemistry is being able to control what product is formed. This can be especially challenging when chirality is introduced. Chiral molecules are commonly sorted into three different groups: central, planar and axial chiral molecules. Central chirality is the most common and is focused on a point (atom) which contains four different groups. Planar chirality involves a chiral plane while axial chirality contains a chiral axis. For all types of chirality there exist two possible products that can be formed. The difficulty with synthesis when chirality is involved is therefore that typically only one of these products, known as enantiomers, is desired.

\subsection{Enantioselectivity}

Enantiomers are molecules that are mirror images of each other but are nonsuperposable. ${ }^{1}$ This means that they have identical chemical and physical properties because they cannot be distinguished by anything that is not chiral itself i.e. substrates that are achiral. Enantiomers will, however, interact differently with plane-polarised light. The introduction of a chiral species results in the enantiomers interacting differently than when they interact with achiral substances. Enantiomers have identical energies since they cannot be distinguished by achiral substances and subsequently a racemic mixture is difficult to separate. Due to the difficulty in separating enantiomers it is advantageous to employ a synthetic strategy in which one enantiomer is formed preferentially; a process otherwise known as enantioselectivity. 
Enantioselectivity can be controlled experimentally using asymmetric synthesis. Complete control however, in terms of obtaining enantiomerically pure products and universal applicability, has not been achieved and research is on going. ${ }^{2}$ Complete control over the enantioselectivity for any reaction is a powerful contrivance to possess. Especially since there is a potential risk of not having a pure compound. Two examples will be used to illustrate how important control over a reaction is.

A well known example is the drug thalidomide. Thalidomide was used as a morning-sickness treatment during pregnancy. It was soon found to be a teratogen, causing malformation of an embryo, due to the presence of both enantiomers. The (R)-enantiomer exhibited sedative properties whereas the $(\mathrm{S})$-enantiomer was found to exhibit teratogenic effects. ${ }^{3}$ This variation of their properties arises due to the differing configuration of the chiral centre. This illustrates how important the configuration of a chiral centre is. It was, however, discovered that due to an acidic hydrogen at the chiral centre thalidomide would racemize (see Figure 1.1) thus even if enantiopure thalidomide was obtained it would still be harmful.
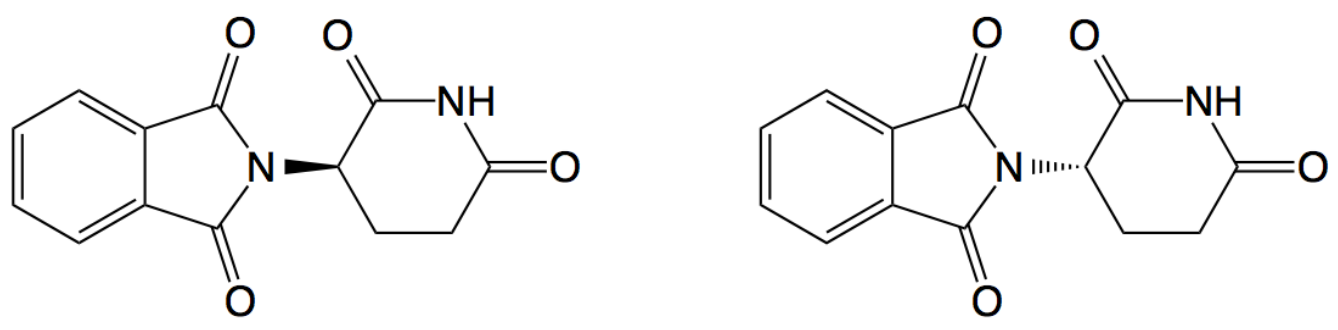

Figure 1.1: Structure of thalidomide. The (S)-enantiomer is shown on the left while the $(\mathrm{R})$-enantiomer is on the right. The chiral centre, on the carbon of the six membered ring bonded to the nitrogen of the five membered ring, contains an acidic hydrogen which leads to racemisation.

The Parkinson's disease treatment drug (L)-DOPA is another case where enantioselectivity is essential. (L)-DOPA is the active form while (D)-DOPA is currently believed to be biologically inactive (Figure 1.2). The presence of (D)-DOPA requires the dosage to be increased to achieve the same results as a drug containing pure (L)-DOPA. This results in unwarranted wastage of materials. As well as this, excessive amounts of (D)-DOPA in the human body could prove to have adverse effects. Either a synthetic route is needed so that only (L)-DOPA is synthesised 
or a resolution method is needed to separate the enantiomers, such as through the formation of diastereomers.<smiles>N[C@@H](Cc1ccc(O)c(O)c1)C(=O)O</smiles><smiles>N[C@@H](Cc1ccc(O)c(O)c1)C(=O)O</smiles>

Figure 1.2: Structure of (L)-DOPA, left, and (D)-DOPA, right. (L)-DOPA is a Parkinson's disease treatment drug while its enantiomer (D)-DOPA is biologically inactive.

\subsubsection{Diastereomers}

Molecules that contain more than one chiral centre can also be diastereomeric. Diastereomers contain more than one chiral centre and have a different configuration at one or more, but not all, chiral centres. ${ }^{1}$ Unlike enantiomers diasteromers are not equal in energy as they are not mirror images of each other. Consequently they can be separated with relative ease. Resolution methods that rely on the formation of diasteromers can, however, be expensive and wasteful since the unwanted stereoisomer must be disposed of. On the other hand a synthetic route requires an enantiomerically pure starting material which is only ideal if it is readily available and affordable. Both methods to obtain enantiomerically pure products still need to undergo further development. Much of the literature is focused on exactly that; finding new resolution methods ${ }^{4}$ and developing better synthetic routes. ${ }^{5}$

Both synthetic routes and resolution methods rely on diastereomers having different energies. Enantioselective reactions rely on the formation of diastereomers, either in the form of diastereomeric intermediates or diastereomeric transition states, to create an energy difference that allows for their separation. This energy difference leads to a thermodynamic or kinetic preference respectively in the formation of one diastereomer over the other.

During a reaction there will be a kinetic preference for one diastereomer only when the transition states are different in energy. If, for example, the transition state for 
the $(\mathrm{R})$-containing diastereomer is lower in energy this reaction will occur much faster than that with the $(\mathrm{S})$-containing diastereomer. This means the reaction is under kinetic control. Consequently the $(\mathrm{R})$-containing diastereomer will be formed preferentially if the temperature is kept low and the reaction time is kept short.

Unlike with kinetic control, thermodynamic control for a reaction with enantiomers is only possible if a new chiral centre is formed and thus leads to diastereomers. This is because two possible products can be formed for each enantiomer; containing either a $(\mathrm{R})$ or $(\mathrm{S})$ chiral centre. There will be a thermodynamic preference for one because the resulting diastereomer will be lower in energy. If no chiral centre is formed there can be no thermodynamic preference because only one product is able to be formed. Even if one enantiomer leads to a lower energy diastereomer the configuration of the already present chiral centre would have to invert for it to form the more thermodynamic product. As such thermodynamic control can only be used to dictate the configuration of a new chiral centre when diastereomers are formed.

The enantiomers are released in a subsequent reaction with one having been formed in a higher amount. By knowing when diastereomers arise in a reaction their energies can ideally be manipulated. As such the energy difference between these diastereomers can be increased thereby amplifying the amount of major product formed. Ideally complete control would be sought so that the energies of the diastereomers can be reversed. This would allow access to the usual minor product in high yields.

\section{$1.2 \quad$ Asymmetric Synthesis}

Asymmetric synthesis is a method that allows the formation of one enantiomer over another. It is a chemical reaction where one or more new elements of chirality are added to a molecule and the stereoisomers are produced in unequal amounts. ${ }^{1}$ Asymmetric synthesis is the most commonly used method to control which enantiomer forms in a reaction. This method requires the starting material to be prochiral; containing no chiral centres but becoming chiral with one substitution or addition. Prochiral starting materials are typically aldehydes, ketones and alkenes (Figure 1.3). 

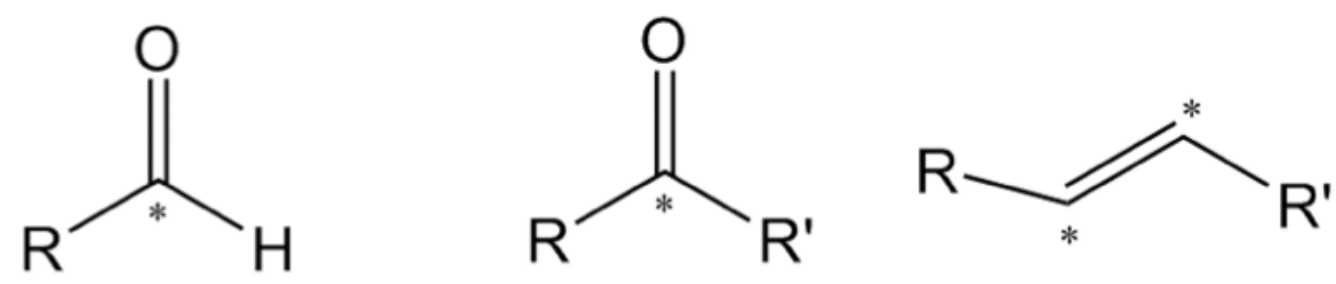

Figure 1.3: The structure of three different generic molecules (aldehyde, ketone, alkene) is shown which all contain an enantiotopic face. The asterisk indicates where attack of the enantiotopic face would occur by a nucleophile. Due to the planar shape at this carbon the nucleophile is able to attack from above and below thus creating two different chiral centres.

During an asymmetric reaction the enantioselectivity is controlled by preferentially reacting with one enantiotopic face or group of the prochiral substrate. A face of a molecule is enantiotopic when attack of the face results in the formation of a chiral centre. For example all three structures in Figure 1.3 have enantiotopic faces because attack on one side would result in one enantiomer while attack of the other side would result in the other enantiomer. An enantiotopic group is when one of two identical groups is replaced by a non-identical group i.e. a group differing to those on the prochiral carbon. This results in the formation of a chiral centre. Figure 1.4 depicts a molecule where substitution of one of the hydrogens with another group, such as a halogen, would result in the carbon becoming chiral. Addition of a non-identical group to an enantiotopic face results in the molecule becoming chiral. Comparatively substitution of an enantiotopic group for a nonidentical group results in the molecule becoming chiral.
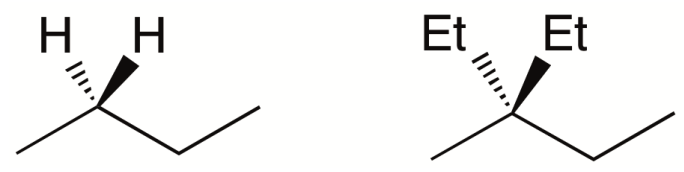

Figure 1.4: The structure of two molecules (butane and 3-ethyl-3methylpentane) is shown which contain enantiotopic groups, the hydrogens and ethyl groups indicated respectively. Substitution of these groups with a nonidentical group arises in the formation of enantiomers.

To have control over the enantioselectivity a non-racemic asymmetric component is needed in the reaction. Without a source of asymmetry, the transition states 
and intermediates that lead to the product will be enantiomeric. Since their energies are equal a racemic mixture results. The asymmetric component ensures that the transition states and intermediates become diastereomeric since enantiomers interact differently with asymmetric substances. The stereoisomer with the lower energy transition state will therefore form preferentially meaning high enantioselectivities are generally obtained. The asymmetric component in a reaction can be a chiral non-racemic solvent, reagent, catalyst or substrate.

\subsubsection{Chiral non-racemic solvent}

The majority of reactions that are conducted in chemistry are carried out in a solvent. Using a solvent that is chiral ensures that all components of the reaction are surrounded by an asymmetric portion. This is because the solvent interacts with everything in the system. As such the enantiomers are continuously surrounded by an asymmetric component and therefore the transition states and intermediates are all affected. Consequently a strongly coordinating chiral non-racemic solvent can make enantiomeric transition states and intermediates diastereomeric so that one stereoisomer forms preferentially.

A chiral amino ether solvent was first used in 1975 by Seebach et al. in the electrodimerisation of ketones. An average optical yield of $6 \%$ was achieved. The optical yield indicates how the optical purity of the product compares to that of the solvent. The use of the chiral solvent preferentially formed one enantiomer but the selectivity was low. ${ }^{6}$

For a chiral solvent the selectivity relies on the way the solvent is oriented. This is unpredictable, however, and thereby leads to low selectivities. One of the main problems with using a chiral solvent is their high cost and difficult synthesis. The solvent must be enantiomerically pure otherwise no control would be obtained and a racemic mixture would form. Synthesising such a large quantity of enantiomerically pure solvent is difficult and expensive. Currently there has been interest in chiral ionic liquids due to their relative ease of synthesis. ${ }^{7,8}$ 


\subsubsection{Chiral non-racemic reagent}

An alternative to surrounding the entire reaction with an asymmetric component is to use a reagent. Here the reagent that is required in the reaction to procure the product is the asymmetric component. This ensures that only the preferred enantiomer is formed. Since the reagent is essential in the reaction it is an ideal point to introduce asymmetry. Only a stoichiometric amount is needed compared to the large amount of solvent that is needed in a reaction. Since the reagent is involved in the transition state these become diastereomeric as a result of the chirality present in the reagent.

Kaufmann and co-workers used a binaphthyl boronate to selectively attach an allyl group to an aldehyde. The selectivity was controlled by the formation of a six-membered ring in the transition state (see Scheme 1.1). The (S)-enantiomer was obtained in a yield of $92 \%$ and an ee of $88 \%$. Upon the addition of dilute hydrochloric acid the allylic alcohol was retrieved. It was also possible to recover binaphthol, which was subsequently recycled to binaphthyl borate. This recycling process reduced the amount of waste and the cost. ${ }^{9}$

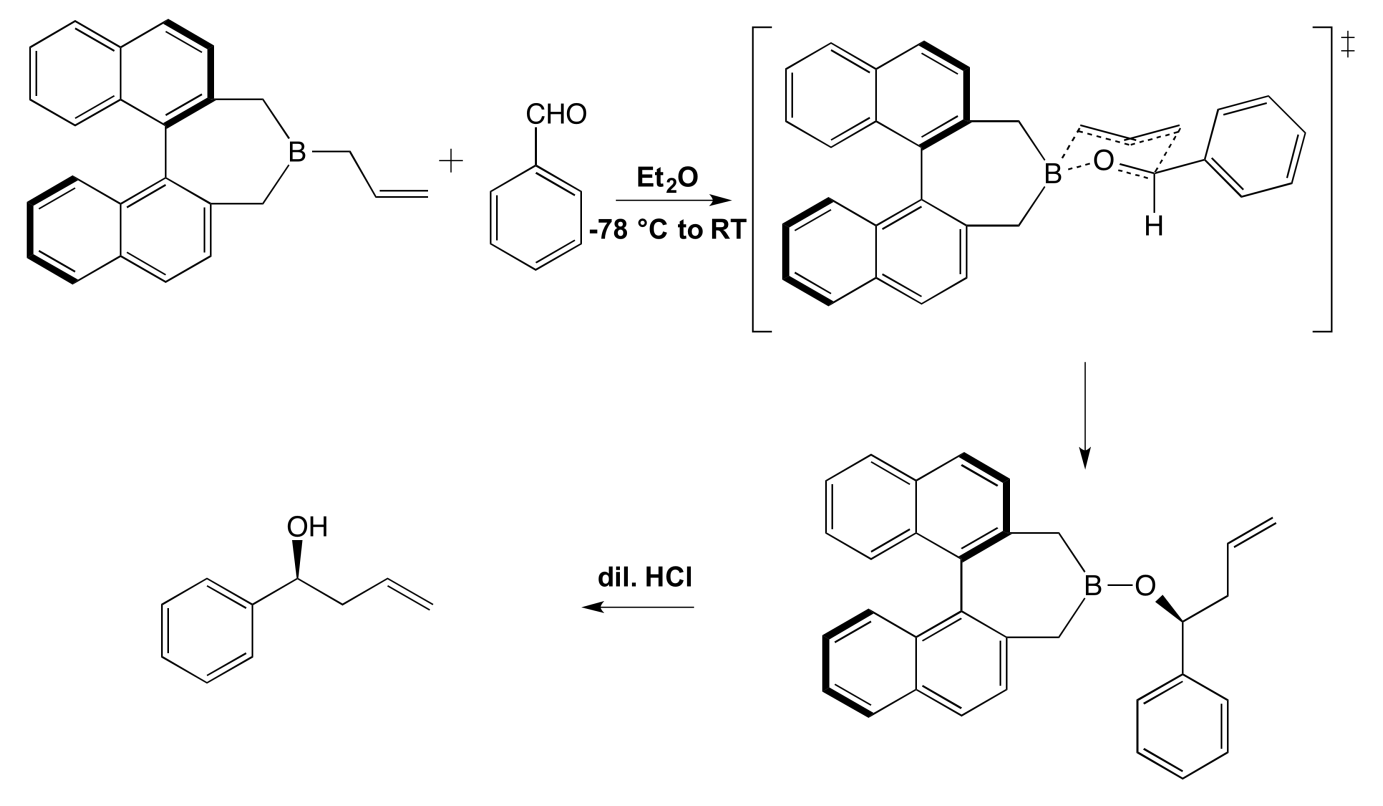

SCHEME 1.1: Reaction scheme showing the use of a chiral boronate reagent in asymmetric synthesis. The other enantiomer of the product can be synthesised using the other form of the reagent.

This reaction showed the success of a chiral boronate in asymmetric synthesis. This is especially true in comparison to the use of a chiral solvent. The success 
of the reagent was thought to be due to the bulk and rigidity of the chiral ligand. Since the ligand is large and unable to rotate throughout the reaction the substrate and reagent are only able to approach each other in one orientation. Only one enantiomer can thereby be formed. This is because the transition state is lower in energy when forming the enantiomer that interacts favourably with the reagent. The transition state leading to the other enantiomer is higher in energy because the reagent and the two substrates come into an unfavourable orientation. The other enantiomer can therefore be produced by using the other configuration of the reagent. Owing to the fact that only a stoichiometric amount of the reagent is needed the waste and cost of this form of asymmetry is more cost effective, especially due to the better selectivity.

In general chiral reagents, such as the binaphyl boronate, show high enantioselectivity and the asymmetric portion is generally recyclable. ${ }^{10,11} \mathrm{~A}$ whole equivalent of the reagent is, however, still required which can be expensive and further improvements in the selectivity can still be made.

\subsubsection{Chiral non-racemic catalyst}

A catalyst provides an alternative route for a reaction which is lower in energy but the catalyst is not incorporated in the final product. The substrate is oriented so that the active components are close together. When a catalyst is made chiral through chiral ligands the reagent and substituent can only be oriented one way. A chiral catalyst consequently brings together the reagent and the prochiral substrate in a specific orientation. One form of the catalyst will preferentially result in one enantiomer while the mirror image of the catalyst will give the other. Both enantiomers are thereby easily accessible as long as the catalyst is readily available. The chiral catalyst can usually be recovered and recycled. Typically only a small amount of the catalyst is needed, compared to the substrate. The application of catalysts in asymmetric synthesis are limited due to the difficulty in synthesising chiral ligands. ${ }^{12}$

Wang investigated the use of a chiral palladium catalyst to asymmetrically hydrogenate dibenzothiazepines. Scheme 1.2 shows the asymmetric hydrogenation of a dibenzothiazepine. The (R)-enantiomer was synthesised in a yield of $91 \%$ and with an $e e$ of $92 \%$. In general the ee of the products ranged from $56 \%$ to $94 \%$. The low selectivity for some of these compounds was owing to the fact that the solvent 
was changed to increase the yield which had a negative effect on the selectivity. This shows the potential use of a chiral catalyst in asymmetric synthesis and how important solvent choice is for these reactions. ${ }^{13}$
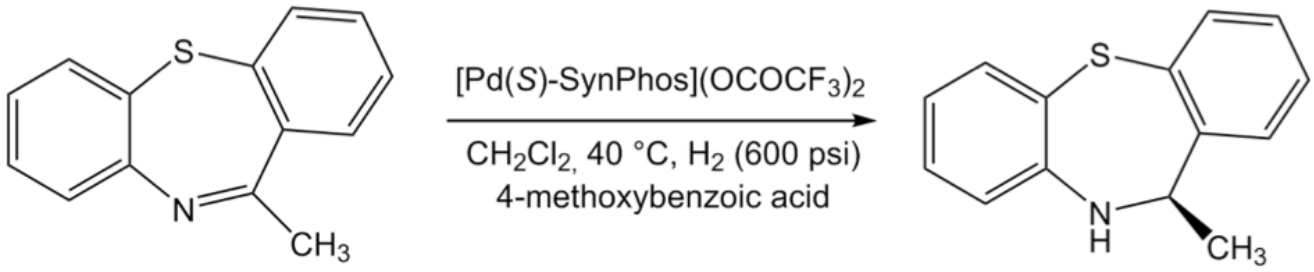

SCHEME 1.2: Asymmetric hydrogenation of a dibenzothiazepine. The chirality of the ligands ensures the formation of one chiral centre.

\subsubsection{Chiral non-racemic substrate}

If a substrate already contains chiral centres these will dictate the configuration of the new chiral centre. This influence of other chiral centres on newly forming ones is utilised with a chiral auxiliary. If no chirality is yet present in the molecule a chiral auxiliary can be used. A chiral auxiliary is the most commonly used chiral non-racemic substrate in asymmetric synthesis. It is a chiral molecule that is temporarily incorporated in a synthetic strategy by attaching it to the prochiral substrate. Thus the selectivity is controlled through the chirality present in the auxiliary. The chiral auxiliary must therefore be attached before the prochiral substrate is made chiral; as shown in Figure 1.5. This means that the chiral auxiliary has to be enantiomerically pure to have control over the enantioselectivity.

The selectivity that is obtained depends greatly on the prochiral substrate used and the chiral auxiliary. As with the catalyst one configuration of the chiral auxiliary will give one stereoisomer while the other configuration will give the opposite stereoisomer. Occasionally a specific stereoisomer is not able to be synthesised because of mismatched effects. If during the reaction groups on the auxiliary and the prochiral substrate come into an unfavourable orientation it can prevent the formation of a product. This is known as a mismatched effect. If the orientation is favourable and leads to a product it is known as a matched effect. 


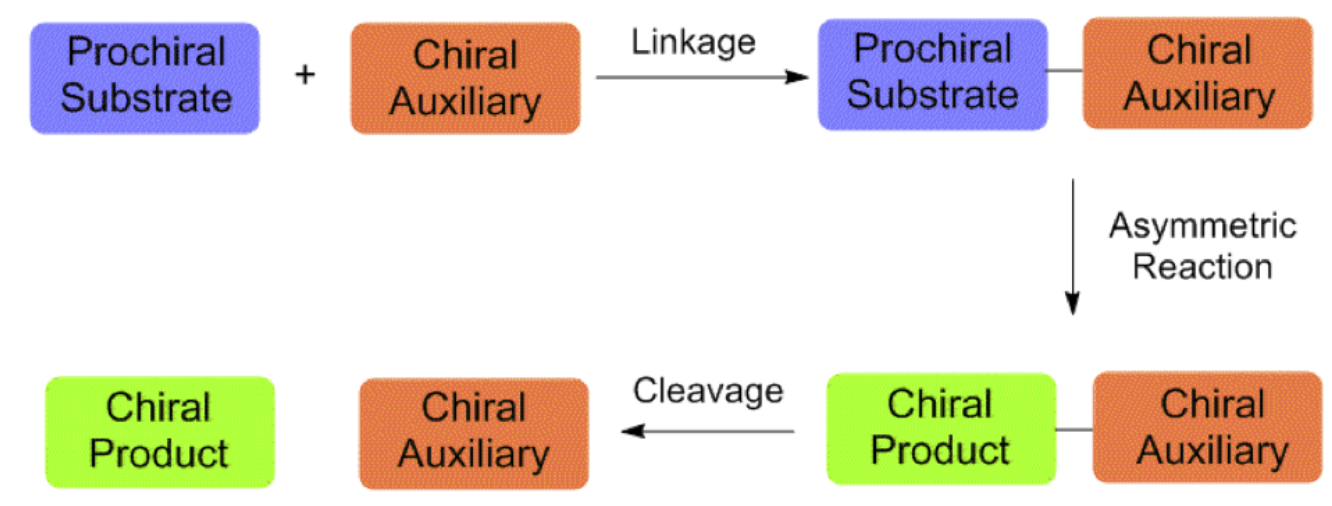

Figure 1.5: General method for using a chiral auxiliary. The chiral auxiliary is attached to the prochiral substrate in a 'Linkage' step. This is followed by the 'Asymmetric Reaction'. Consequently the prochiral substrate becomes chiral and the chiral auxiliary is removed in a 'Cleavage' step.

Since an auxiliary is chiral the product formed after the addition of chirality to the prochiral centre will be diastereomeric. Since both diastereomers are formed these can then be separated due to their differing energies. This can be done using chromatography, crystallisation in a solvent where only one diastereomer is soluble, or a combination of the two. Once separated the chiral auxiliary is then cleaved to give the new chiral product with high purity. Typically the auxiliary is recovered and can be reused without degradation.

The chemistry of chiral auxiliaries is very well understood hence the wide spread usage in the literature. ${ }^{14-16}$ The main disadvantage is that an extra linkage and cleavage step is needed, thus reducing the overall yield. The enantioselectivity depends not only on how well the chiral auxiliary controls which diastereomer forms but also on how easily the diastereomers are separated. The chirality present on the auxiliary must also be close enough to the prochiral substrate to have an affect on which face is attacked in the reaction. Hence the substrate scope is limited.

Bornmann and co-workers synthesised a portion of psymberin by using an Evans auxiliary in the asymmetric aldol reaction. They required an anti-relationship between the hydroxyl and methoxy group hence the use of an Evans auxiliary. The reaction resulted in a yield of $60 \%$ and the anti-selectivity was obtained due to the auxiliary shielding the front face as shown in Scheme 1.3. The front face was shielded on account of the tin coordinating to the aldehyde oxygen and the 
carbonyl oxygen in the auxiliary. This resulted in the auxiliary realigning itself to reduce the net dipole of the molecule. The aldehyde therefore approached from the back face giving the desired anti-relationship. The psymberic ester was synthesised with an $e e$ of $88 \%$ showing the power of using an auxiliary. ${ }^{17}$<smiles></smiles><smiles>CC(C)[C@H]1COC(=O)N1C(=O)COCc1ccccc1</smiles>

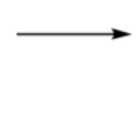

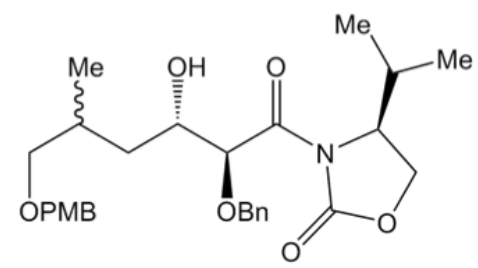

Scheme 1.3: Reaction scheme showing the synthesis of a portion of psymberin.

An anti-relationship was obtained in the product due to the Evans auxiliary shielding the front face from attack.

\subsection{Cyclophanes}

A cyclophane is a molecule that typically consists of two benzene rings held face to face by methylene bridges. ${ }^{18}$ The cyclophane backbone is chemically stable towards oxidation, acids, bases and light. It is also very rigid and stable at relatively high temperatures.

Two examples are shown in Figure 1.6. Cyclophanes are named according to the length of the methylene bridges and the relative attachment on the benzene ring using the prefix ortho, meta, or para. Ortho and meta cyclophanes require more than two methylene bridges to ensure the rings are aligned i.e. on top of each other.

One of the most commonly used cyclophanes is [2.2]paracyclophane (Figure 1.7). This was discovered in 1949 by C. J. Brown and A. C. Farthing through a polymerization reaction starting from para-xylene. The authors named this product di-p-xylylene and determined its structure using X-ray diffraction. They found that the bond lengths for the chain carbons within in the crystal where 1.5, 1.6 and $1.5 \AA$ while the carbon bond lengths in the benzene rings were all $1.4 \AA$. The benzene rings however were not planar because the two carbons in the ring that are attached to the carbon chains were displaced from the plane of the ring by $0.1 \AA$. They believed this to be to reduce strain in the molecule. The other four 

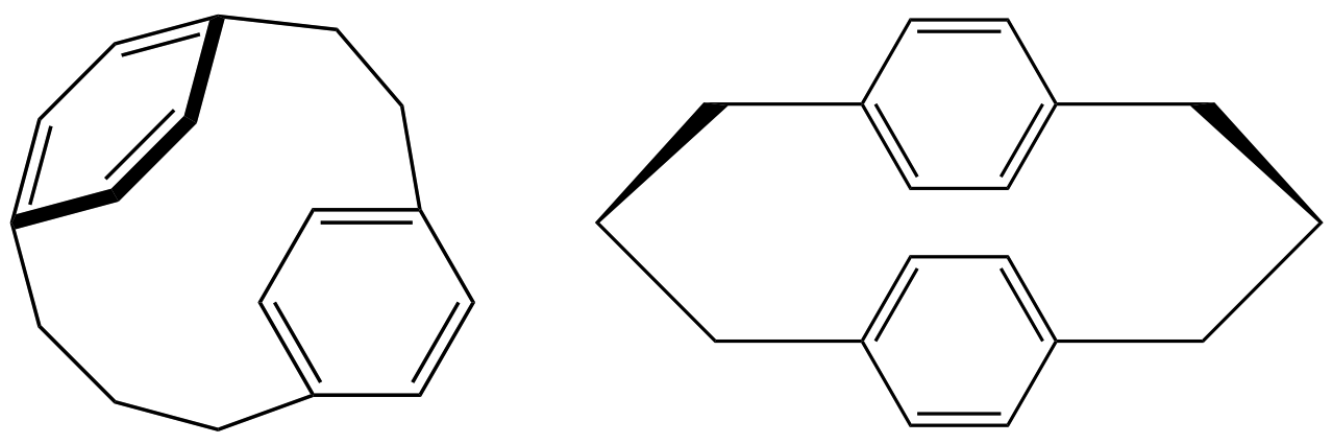

FIgURE 1.6: The structure of two paracyclophanes is shown. [3.2]paracyclophane on the left and [3.3]paracyclophane on the right.

carbon atoms were a distance of $3.1 \AA$ from the ring below. The distance between the substituted carbons of the ring was only $2.8 \AA$. The bond angle between the carbon chains was $115^{\circ} .{ }^{19}$

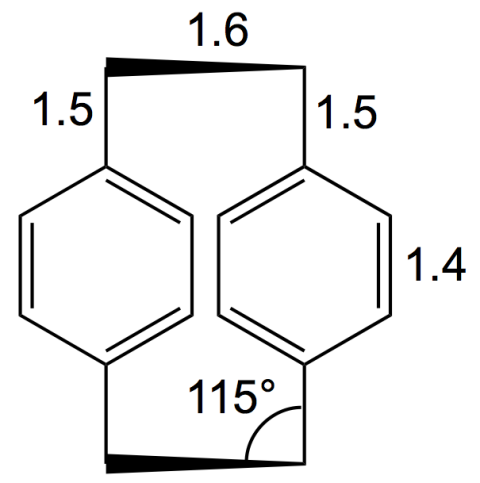

FIgURE 1.7: The structure of [2.2]paracyclophane. This was discovered in 1949 and then first purposefully synthesises in 1951. The two phenyl rings are arranged on top of each other. Distances are shown in Angstroms.

It was then first purposefully synthesized in 1951 by D. J. Cram and H. Steinberg starting from benzyl chloride. Many different cyclophanes with varying chain lengths were synthesised. The authors suggested the name paracyclophane for these compounds because of the para attachment of the carbon chains (Figure $1.7) .^{20}$

Substitution with an achiral substituent at one of the carbons causes the cyclophane to become chiral. Substitution at a ring carbon give rise to planar chirality. A molecule exhibits planar chirality when it contains a chiral plane instead of a chiral point. Substituted cyclophanes exhibit planar chirality because they do not 
contain an asymmetric carbon but a chiral plane. The notation $\left(\mathrm{S}_{p}\right)$ and $\left(\mathrm{R}_{p}\right)$ is used to distinguish between the two enantiomers where the ' $p$ ' refers to the fact they exhibit planar chirality. The configuration is determined by first identifying the chiral plane in the cyclophanes, which is the plane containing the most atoms. A pilot atom is then chosen; the closest atom to the plane with the highest priority according to the Cahn-Ingold-Prelog rules. The three closest atoms to the pilot atom in the plane are then labeled 'a', 'b' and 'c' along one path. If there is a choice of paths the one containing the higher priority atom is chosen. By then determining whether the path from the pilot atom to ' $\mathrm{c}$ ' is clockwise or anticlockwise the notation $\left(\mathrm{R}_{p}\right)$ and $\left(\mathrm{S}_{p}\right)$ is used respectively. ${ }^{21}$

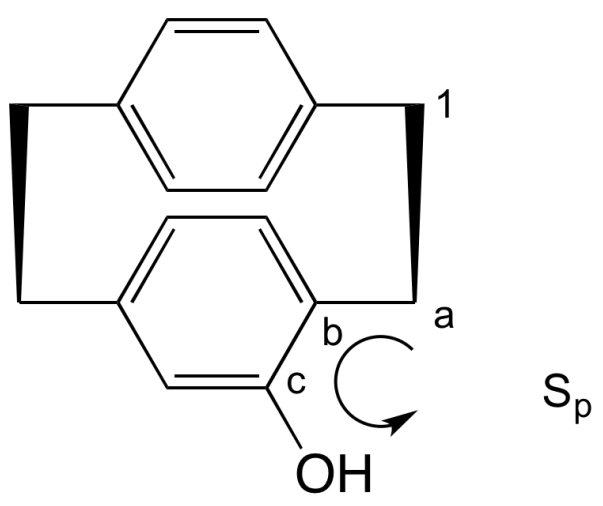

FiguRE 1.8: Determining the configuration of a planar chiral molecule. The pilot atom is chosen, $\mathbf{1}$, and a path leading to the higher priority group is determined. The path traced out by moving from the pilot atom through $a, b$ and finally $\mathrm{c}$ is anti-clockwise hence this is the $\left(\mathrm{S}_{p}\right)$-enantiomer.

For example the chiral plane for the molecule in Figure 1.8 is the one containing the hydroxyl group. The pilot atom is the carbon labeled 1. The hydroxyl group has the highest priority and the path will lead in that direction. The carbon part of the methylene bridge is the first atom on the path and is designated with the letter ' $a$ '. The carbon in the ring is designated ' $b$ ' and the hydroxyl group ' $c$ '. This molecule is therefore the $\left(\mathrm{S}_{p}\right)$-enantiomer since the rotation, from the point of view of the pilot atom, is anticlockwise.

Substituted cyclophanes are used as chiral auxiliaries and chiral ligands due to their relative ease of synthesis. Scheme 1.4 shows four reactions which lead to four different chiral [2.2] paracyclophanes which are then resolved in a separate step. These molecules can then be further reacted to obtain cyclophanes containing different functionalisation such as a carboxylic acid or amine group. 

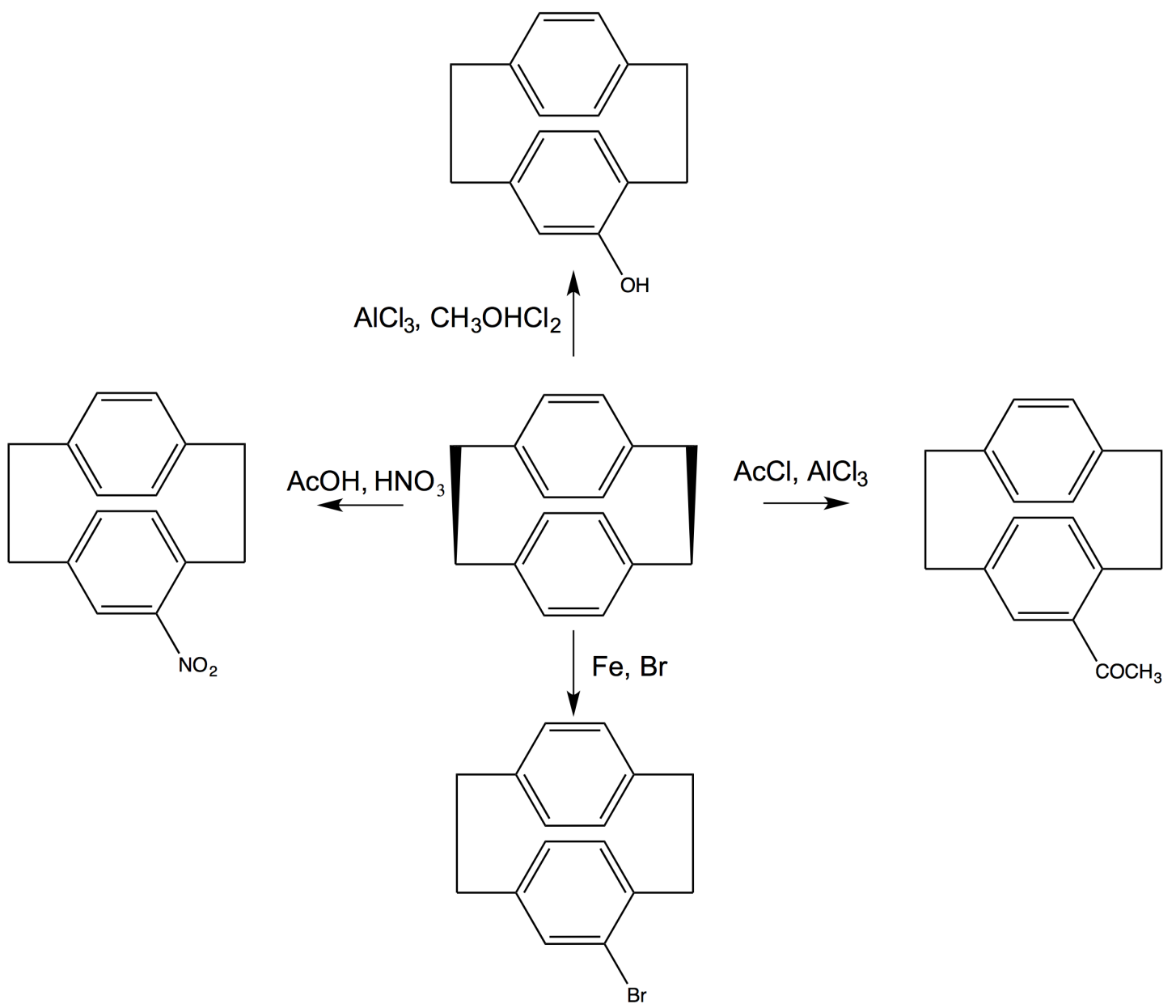

Scheme 1.4: Reaction schemes showing some of the monosubstituted derivatives that can be synthesised from [2.2]paracyclophane.

Disubstituted [2.2]paracyclophanes are typically formed from hydroxy-[2.2]paracyclophane by the addition of a formyl group ortho to the hydroxyl group. The two functional groups on the cyclophane can be on the same ring or on different rings. Scheme 1.5 shows the use of a tin catalyst in the addition of a formyl group. This compound is known as 4-formyl-5-hydroxy[2.2] paracyclophane (FHPC) and can then be further functionalised to give other disubstituted cyclophanes. ${ }^{21}$

[2.2] paracyclophane derivatives have many applications across a variety of different areas in chemistry. They have been used in areas such as polymer science ${ }^{22}$, and in catalysis. ${ }^{21,23,24}$ They have also been used in the design of new optoelectronic materials, ${ }^{25}$ and electron transfer processes. ${ }^{26,27}$ Functionalised [2.2] paracyclophanes are used in asymmetric synthesis due to the fact that they exhibit planar chirality. [2.2]paracyclophane derivatives have been used in asymmetric synthesis as both 
chiral auxiliaries ${ }^{28,29}$ and chiral ligands. ${ }^{30,31}$ Current research has focused on the synthesis of new chiral ligands incorporating the [2.2]paracyclophane backbone. As much of the current research is focused around chiral ligands this field is particularly diverse. Subsequently it will be discussed in the following subsection. Two examples of a [2.2]paracyclophane derivative used as a chiral auxiliary will be briefly mentioned here.
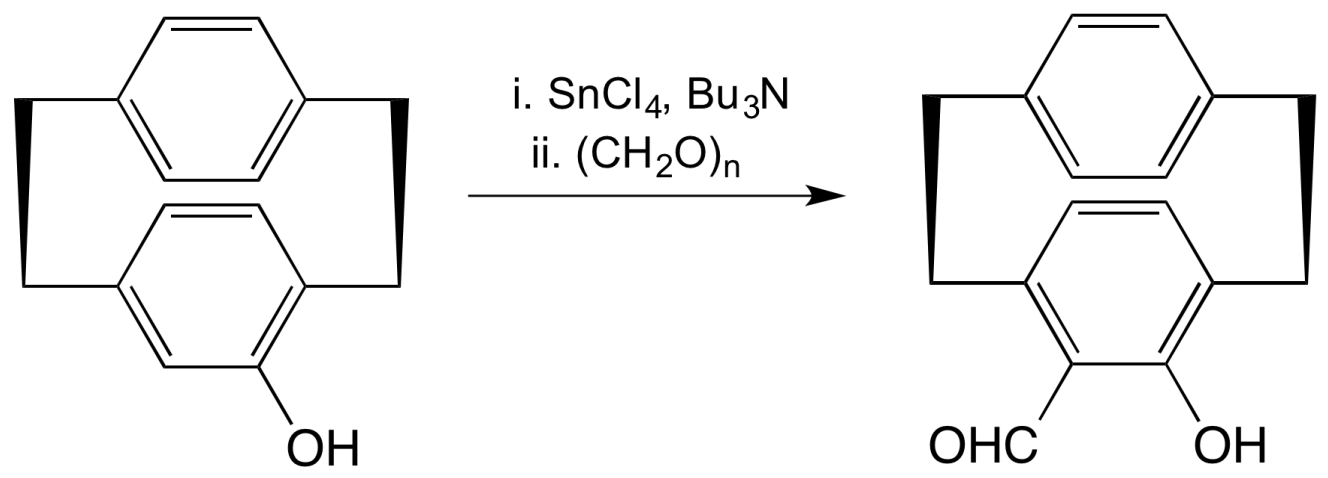

Scheme 1.5: Reaction scheme for the synthesis of FHPC, a disubstituted derivative of [2.2]paracyclophane.

Chiral auxiliaries should provide a suitable chiral environment and have a rigid side chain to give high stereoselectivity therefore researchers are trying to find the ideal chiral auxiliary. Planar chiral paracyclophanes have been used in reactions such as: Diels-Alder, ${ }^{32}$ aldol, ${ }^{33}$ Michael addition, ${ }^{32}$, boration, ${ }^{34}$ addition, ${ }^{35}$, and the copper-catalysed Henry reaction ${ }^{36}$.

Molecules that exhibit planar chirality have been of interest as chiral auxiliaries. Previously research has focused on using ferrocene derivatives but since the synthesis of PHANEPHOS paracyclophane chemistry has become a growing area. ${ }^{37}$ The main issue with using planar chiral molecules is the fact that they are often very unstable in most reaction conditions. The cyclophane backbone is however very chemically stable. Substituted cyclophanes will also not undergo racemisation until relatively high temperatures, around $200^{\circ},{ }^{38}$ which means that they are ideal planar chiral molecules to be used as chiral auxiliaries.

Soai and co-workers examined the role of monosubstituted [2.2]paracyclophane derivatives in the addition of isopropylzinc. Cyclophane (S)-1a (see Scheme 1.6) resulted in the (R)-enantiomer in 91\% yield and 95\% ee. Cyclophanes (S-)1b and (S)-1c gave similar results with yields of around $95 \%$ and $e e$ 's of about $94 \% .{ }^{29}$ This shows the great potential of [2.2]paracyclophane derivatives as chiral auxiliaries. 

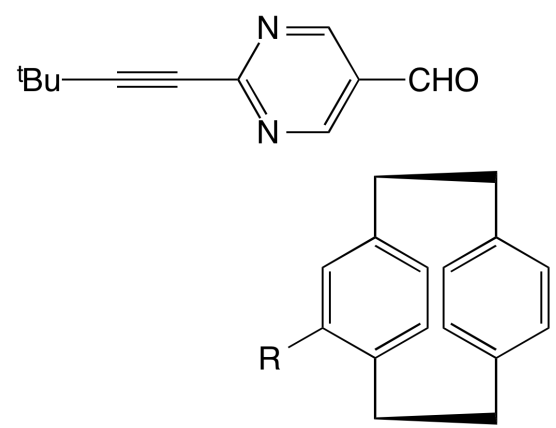

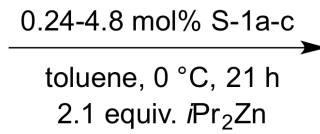

$\mathrm{S}-1 \mathrm{a} \mathrm{R}=\mathrm{COOH}$

$\mathrm{S}-1 \mathrm{~b} R=\mathrm{Ac}$

$\mathrm{S}-1 \mathrm{c} \mathrm{R}=\mathrm{COOMe}$

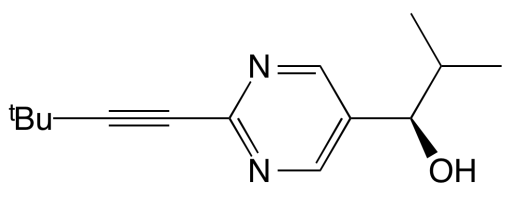

SCHEme 1.6: Reaction scheme for the addition of isopropylzinc using a monosubstituted [2.2] paracyclophane derivative as a chiral auxiliary.

In 1994 Belokon and co-workers investigated the use of a [2.2]paracyclophane derivative as a chiral auxiliary. The derivative was 4-formyl-3-hydroxy[2.2]paracyclophane (FHPC) (Figure 1.9), an analogue of salicylaldehyde. FHPC was synthesised by preferentially introducing a hydroxyl group to one of the rings. This was followed by the addition of a formyl group ortho to the hydroxyl. The hydroxyl group was introduced by the addition of $\mathrm{Br}_{2}$ to give the brominated product, followed by a Li-Br exchange using BuLi, reaction with trimethylborate, $\mathrm{B}\left(\mathrm{OCH}_{3}\right)_{3}$, and finally oxidation with $\mathrm{H}_{2} \mathrm{O}_{2}$. The formyl group was added using $\left(\mathrm{CH}_{2} \mathrm{O}\right)_{n}$ catalysed by $\mathrm{SnCl}_{4}$ and $\mathrm{Bu}_{3} \mathrm{~N}$ to give a racemic mixture of FHPC (Scheme 1.7). This was then separated through the formation of diastereomeric Schiff bases, which will be discussed further in the 'Resolution Methods' section. ${ }^{28}$
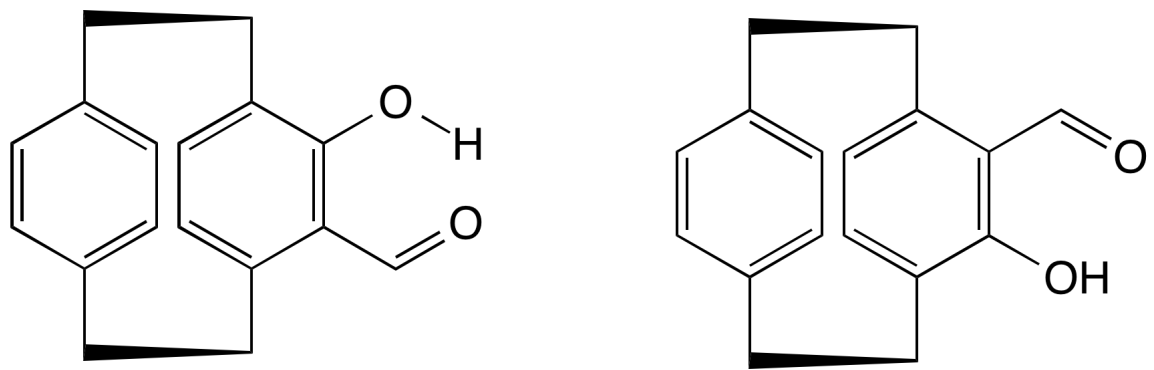

FiguRE 1.9: Structure of both enantiomers of FHPC. $\left(\mathrm{S}_{p}\right)$-FHPC is shown on the left and $\left(\mathrm{R}_{p}\right.$-FHPC) is shown on the right.

Non-racemic FHPC was then used to synthesise $\beta$-hydroxy- $\alpha$-amino acids. This was done by first reacting the auxiliary with glycine and copper(II) to give the coordinated imine. The alpha position of the carbonyl was then deprotonated to give the enolate which further reacted with either isobutyraldehyde or benzaldehyde to give two different amino acids. Scheme 1.8 shows the reaction with 
benzaldehyde. Upon the addition of $\mathrm{HCl}$ the chiral auxiliary was cleaved giving the isolated amino acid with enantiomeric purity greater than $90 \%$. The syn:anti ratio was on average 20:1 but for the reaction involving benzaldehyde this could not be measured due to the small amount of anti product. FHPC was recovered almost quantitatively and without degradation. ${ }^{28}$<smiles>c1ccc(CCc2ccc(CCc3ccccc3)cc2)cc1</smiles>

$$
\begin{aligned}
& \text { i. } \mathrm{Br}_{2} \\
& \stackrel{\text { ii. } \mathrm{BuLi}}{\longrightarrow} \\
& \text { iii. } \mathrm{B}\left(\mathrm{OCH}_{3}\right)_{3} \\
& \text { iv. } \mathrm{H}_{2} \mathrm{O}_{2}
\end{aligned}
$$
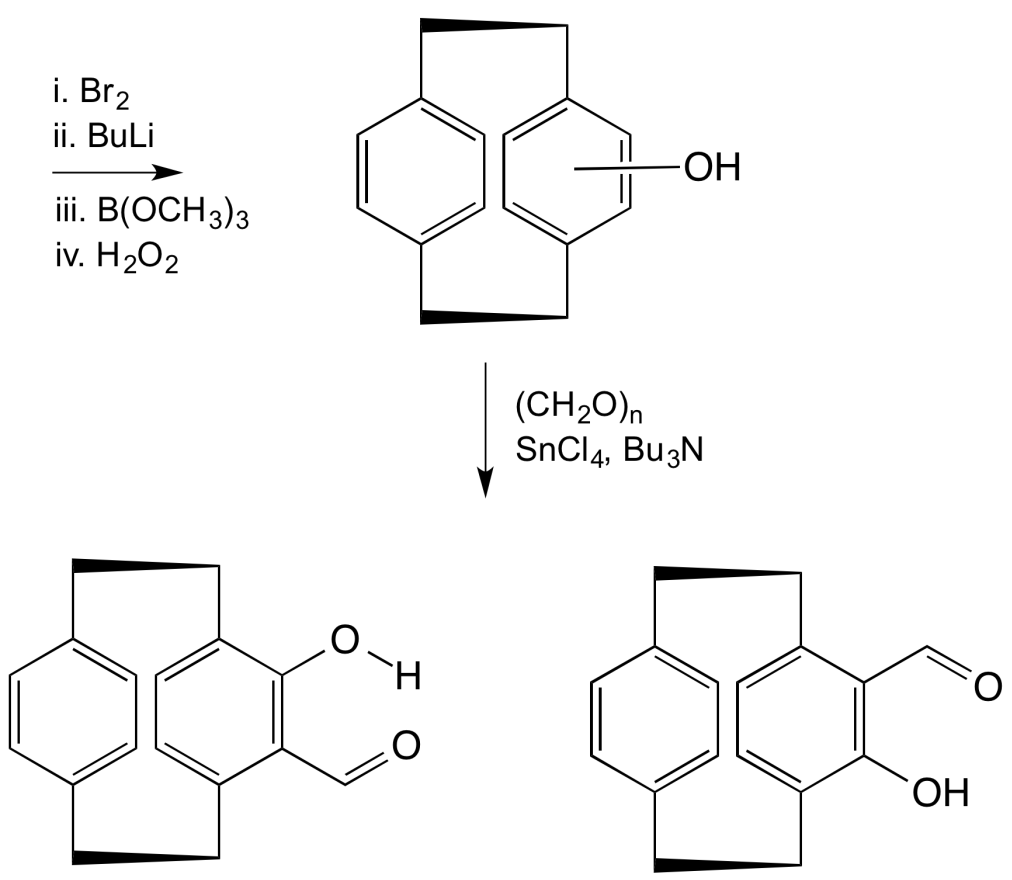

SCHEME 1.7: Reaction scheme showing the synthesis of racemic FHPC starting from [2.2]paracyclophane.

Further work on FHPC was carried out by Antonov et al. because they believed this salicyl aldehyde analog would be useful as a chiral auxiliary or chiral ligand in reactions typically carried out by salicylaldehyde. It had been shown that Schiff bases of chiral salicylaldehydes and chiral amines can be used for asymmetric propanation, oxidation of sulfides, epoxidation of olefins and silylcyanation of aldehydes. $\mathrm{Cu}(\mathrm{II})$ complexes of these Schiff bases have been used for the stoichiometric synthesis of racemic $\beta$-hydroxy- $\alpha$-amino acids and racemic $\alpha$-amino acids. FHPC was synthesised and resolved by forming their corresponding Schiff base's using (R)- and (S)- $\alpha$-PEAM. The resolved enantiomers were then used as chiral auxiliaries for the asymmetric synthesis of $\beta$-hydroxy- $\alpha$-amino acids and $\alpha$-methylphenylalanine; ee's ranged from 45 to $98 \%$. ${ }^{39}$ 


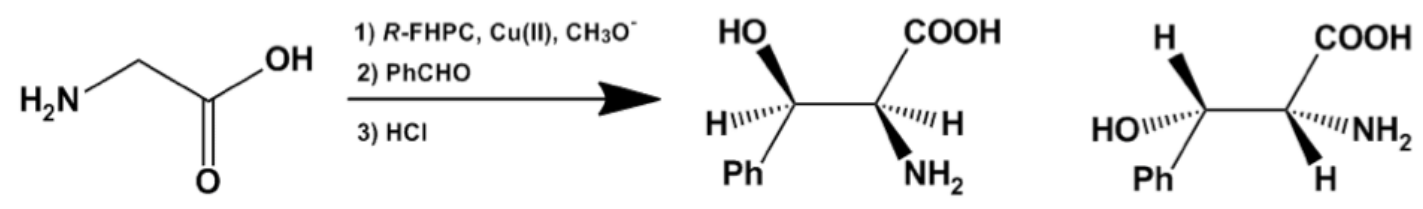

SCHEME 1.8: Reaction scheme of the synthesis of glycine using $\left(\mathrm{R}_{p}\right)$-FHPC as a chiral auxiliary.

\subsubsection{Ligands}

There are a myriad of chiral ligands that have been synthesised starting from [2.2] paracyclophane. ${ }^{31,40-49}$ This section will provide an overview of the diversity of these ligands. This is not intended to be an indication of the entirety of [2.2]paracyclophane chiral ligand synthesis. Nor does this necessarily reflect the weighting of various areas of current research. Instead it aims to provide a broad view of the research currently being undertaken.

Planar chirality is becoming increasingly important in modern organic chemistry. ${ }^{50}$ This is because of the high selectivity that was observed when PHANEPHOS (see Figure 1.10) was used in the hydrogenation of olefins. ${ }^{47}$ Planar chiral [2.2]paracyclophane based ligands contain a rigid [2.2] paracyclophane unit which provides a versatilve platform for designing different types of chiral ligands such as phosphines $^{30,51}$, imidazoliums ${ }^{52}$, carbazoles ${ }^{53}$, imine ligands ${ }^{38,49,54}$, thiol containing ligands $^{55}$, amino thioureas ${ }^{33}$, salen and hemisalen ligands ${ }^{56}$, and isocyano ligands ${ }^{57}$.
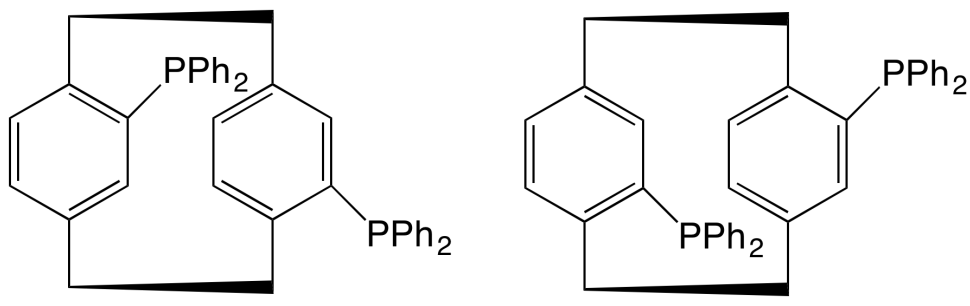

Figure 1.10: The structure of the first substituted [2.2]paracyclophane ligand, PHANEPHOS, is shown. The $\left(\mathrm{S}_{p}\right)$-enantiomer is shown on the left and the $\left(\mathrm{R}_{p}\right)$-enantiomer is shown on the right.

[2.2] paracyclophanes have been used as carbene precursers in the literature. Duan et al. investigated the use of [2.2] paracyclophane in N-heterocyclic carbenes due to the cyclophanes bulk and rigidity. The cyclophanes bulk was desirable because 
an increase in the bulk of a chiral group typically leads to increased enantioselectivities in asymmetric synthesis. The rigidity of the backbone also opens up the opportunity for designing different types of chiral ligands. Duan et al. synthesised a variety of planar chiral N-heterocyclic carbene precursors with restricted flexibility. Application of these ligands resulted in yields between $72-91 \%{ }^{58}$

On going research is being done to designing and synthesising new ligands for the $\mathrm{Cu}(\mathrm{I})$-catalysed asymmetric $\beta$-boration that will increase the substrate scope and selectivity. The products of this reaction are versatile intermediates in organic synthesis and can easily be transformed into important building blocks for pharmaceuticals and natural products. Zhao et al. carried out a copper-catalysed asymmetric conjugate boration using a planar and central chiral bicyclic triazolium ligand. It was found that the use of a strong base such as $\mathrm{KO}^{t} \mathrm{Bu}$ and $\mathrm{NaO}^{t} \mathrm{Bu}$ increased the reactivity but caused a slight decrease in the selectivity compared to $\mathrm{Cs}_{2} \mathrm{CO}_{3}$. While using a weak base such as $\mathrm{CH}_{3} \mathrm{COONa}$ and $\mathrm{CsF}$ showed reduced reactivity and selectivity compared to $\mathrm{Cs}_{2} \mathrm{CO}_{3}$. Different solvents were also tested but it was found that toluene gave the best yield and ee of $94 \%$ and $99 \%$ respectively with the base $\mathrm{Cs}_{2} \mathrm{CO}_{3}$. It was found that the use of one diastereomer, $(\mathrm{S}$, $\mathrm{S}_{p}$ ), of one of theirs ligands resulted in excellent selectivity of $99 \%$ while the use of the other diastereomer, $\left(\mathrm{S}, \mathrm{R}_{p}\right)$, showed a decrease in the selectivity of $70 \%$. A similar ligand which did not contain a hydroxyl group showed a decrease in the enantioselectivity and showed the importance of the hydroxyl group in the catalytic process. A ligand without [2.2] paracyclophane was also investigated and only showed moderate selectivity. Thus showing the importance of the planar chirality of the cyclophane. ${ }^{34}$

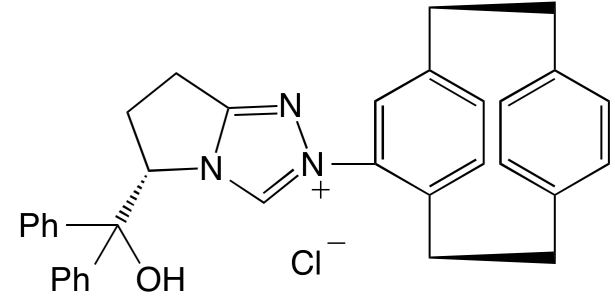

$\left(S, S_{p}\right.$

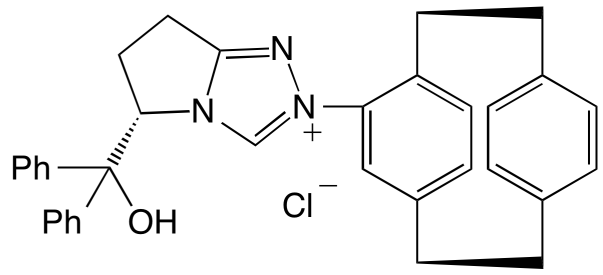

$\left(\mathbf{S}, \mathbf{R}_{\mathrm{p}}\right)$

Figure 1.11: The structure of two diastereomers is shown; $\left(\mathrm{S}, \mathrm{S}_{p}\right)$ and $\left(\mathrm{S}, \mathrm{R}_{p}\right)$. These were utilised in a copper-catalysed asymmetric conjugate boration. 
Examples of planar chiral [2.2]paracyclophane ligands used in copper catalysed reaction in the literature have only become prominent in the last few years. Xin et al. designed and synthesised a series of new planar and central chiral ligands based on the [2.2] paracyclophane backbone. ${ }^{36}$ These were utilised in a coppercatalysed asymmetric Henry reaction to give secondary alcohols. High yields were obtained along with excellent selectivity. It was found that ethanol was a better solvent than THF or $\mathrm{CH}_{2} \mathrm{Cl}_{2}$ for the formation of the copper complex. The best stereoselectivity was, however, obtained with $\mathrm{CH}_{2} \mathrm{Cl}_{2}$. Ethanol resulted in faster reaction rates compared to the other solvents but there was no selectivity observed. Thus ethanol was chosen for the copper complex formation and $\mathrm{CH}_{2} \mathrm{Cl}_{2}$ for the Henry reaction.

The authors concluded that a strong base was needed to form the reactive copper complexes because triethylamine, a weak base, led to low reactivity. Two equivalents of ${ }^{t} \mathrm{BuOK}$ resulted in a better yield and $e e$. Lowering the amount of base to 1.8 equivalents resulted in increased selectivity while lowering the amount of base further decreased both the yield and selectivity. Introducing an additional chiral centre resulted in even better selectivity, which was to be expected because the great number of chiral centres lock the substrate in a specific orientation. Ligand $\mathbf{L}_{\mathbf{2}}$ gave better selectivity than $\mathbf{L}_{\mathbf{4}}$, which was thought to be due to there being less bulk around the cyclophane for ligand $\mathbf{L}_{\mathbf{2}}$ (see Figure 1.12). ${ }^{36}$

The use of a more sterically hindered amino alcohol resulted in better selectivity. The authors also investigated the use of both planar and central chirality to investigate the cooperative effect. A matched pair gave good selectivity while a mismatched pair gave low selectivity. It was found that the configuration of the product was controlled by the configuration of the planar chiral cyclophane. Furthermore substrates with strong electron-withdrawing groups resulted in better yields and selectivity than electron-donating groups.

Vorontsova et al. synthesised Br-AHPC and Br-BHPC (see Figure 1.13) and reacted them with $\boldsymbol{\alpha}$-phenylethylamine to give the corresponding diastereomeric Schiff bases. ${ }^{35}$ These diastereomers were then resolved. The enantiomerically pure Schiff bases were then used as ligands in the addition reaction of diethyl zinc to benzaldehyde. Both diastereomeric forms of the cyclophanes were tested as chiral inductors in the asymmetric addition of diethyl zinc to benzaldehyde; all showed high asymmetric induction. The Schiff bases derived from Br-BHPC showed better efficiencies than Br-AHPC. The induction ability of Br-AHPC and Br-BHPC 
<smiles>C/C(=N\[C@H](CO)c1ccccc1)c1c(Cl)cccc1CCc1ccc(CCc2ccccc2)c(Br)c1</smiles>

$\mathrm{L}_{2}$

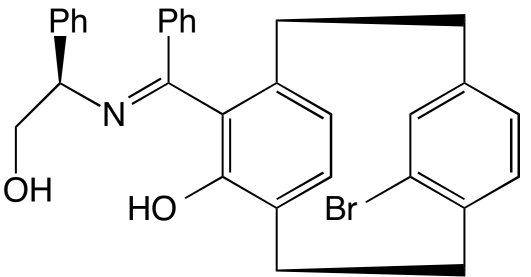

$\mathrm{L}_{4}$

FiguRE 1.12: The structure of two ligands utilised in a copper-catalysed Henry reaction are shown. $\mathbf{L}_{\mathbf{2}}$, on the left, contains an Me group close to the cyclophane whereas $\mathbf{L}_{\mathbf{4}}$, shown on the right, contains a much bulkier phenyl group.

were roughly the same as for their non-bromine counterparts AHPC and BHPC respectively. The addition of a bromine atom inverted the absolute configuration of the product.<smiles></smiles>

$\left(\mathrm{S}_{\mathrm{p}}\right)$-Br-AHPC<smiles></smiles>

$\left(S_{p}\right)-B r-B H P C$

FiguRE 1.13: The structure of the $\left(\mathrm{S}_{\boldsymbol{p}}\right)$-enantiomer of 4-acetyl-13bromo-5-hydroxy[2.2]paracyclophane (Br-AHPC) and 4-benzoyl-13-bromo-5hydroxyl[2.2]paracyclophane (Br-BHPC) are shown.

Aryl amines are important intermediates in synthesis and are often found in natural products but their synthesis is slow and hard. The Hartwig-Buchwald reaction is used to synthesise aryl amines. Kreis et al. carried out a Hartwick-Buchwald addition on a variety of substrates with high diastereoselectivities. ${ }^{50}$ Synthesis of planar and central chiral [2.2]paracyclophane amines was carried out via the Hartwick-Buchwald reaction using electron-rich racemic [2.2] paracyclophane bromide or triflate and a chiral amine. A palladium BINAP catalyst was used for this reaction. For all chiral amines the diastereomer that formed preferentially was independent of the BINAP enantiomer that was used as the ligand. This shows that the product selectivity is superior to the catalyst selectivity. 
The majority of reseach into Pd-catalysed asymmetric allylic alkylation has been focused on the the use of ferrocene derivatives with planar chirality. Substituted [2.2] paracyclophanes have, however, been used since around the 1990s. Jiang et al. aimed to control the structural flexibility and rigidity of ligands by using [2.2] paracylophane-derived P,N-ligands. ${ }^{59}$ These were designed and synthesised and the relationship between the ligands structures and their abilities to induce asymmetry in Pd-catalysed asymmetric allylic alkylations of malonates with 1,3diphenyl 2-propenyl acetate was carried out. High yields and enantioselectivites were seen when ligands with matched planar and central chirality were used.

The authors hypothesised that if the structural rigidity and flexibility could be further controlled then the performance of the ligands could be improved. Six ligands were synthesised in this work. The reaction conditions were varied and the influence of planar and central chirality were explored. It was found that reactions run in $\mathrm{CH}_{2} \mathrm{Cl}_{2}$ gave higher enantioselectivies than those run in THF. Changes in temperature had no effect. It was found that changing the central chirality of the ligand reversed the configuration of the product. The authors showed that planar chirality alone could not give great selectivity and therefore central chirality was also needed to obtain good selectivity. Using the work of Jiang et al. ${ }^{59}$ and Zhao et al. ${ }^{34}$ the importance of both planar and central chirality in a molecule for asymmetric synthesis can be seen.

Hydroxy-based ligands used for asymmetric synthesis and catalysis are some of the most effective chiral inductors. 4-hydroxy[2.2]paracyclophane has been the most common starting point for [2.2] paracyclophane chemistry. The synthesis and resolution of 4-hydroxy[2.2]paracyclophane has attracted a lot of attention due to the fact that it can lead to disubstituted [2.2]paracyclophanes. This can be done through an ortho substitution reaction as was used in the synthesis of FHPC. There have, however, only been a few examples of hydroxy-based pseudoortho ligands. Zhuravsky et al. investigated a new approach to such ligands using bromophenol as the source of chirality. ${ }^{60}$ PHANOL, an analog of BINOL, (see Figure 1.14) was synthesised along with 4-hydroxy[2.2]paracyclophane. These were then resolved into their enantiomers in the hope that they would then be used in asymmetric synthesis.

FHPC is an established precursor to [2.2]paracyclophane Schiff bases, salons and 

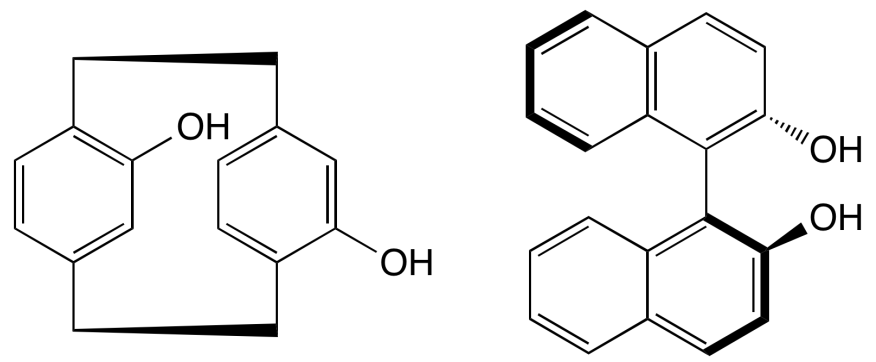

Figure 1.14: The structure of $\left(\mathrm{S}_{\boldsymbol{p}}\right)$-PHANOL (left), a [2.2]paracyclophane analogue of (S)-BINOL (right), is shown.

hydroxyamines, all of which have been used in the asymmetric addition of diorganozinc reagents to aldehydes, sulfoxidation, and trimethylsilylcyanation of benzaldehyde. Iso-FHPC is a regioisomer of FHPC and due to the similarity in the structure is was hypothesised that it could be used in similar reactions.

Bolm et al. synthesised N,O-ligands based on [2.2]paracyclophane to be used in asymmetric synthesis. ${ }^{37}$ These ligands were pseudo-geminal, pseudo-ortho and ortho hydroxy-oxazolinyl[2.2]paracyclophanes. It was found that the substitution pattern had a great effect on the rate and enantioselectivity of the formed catalyst in the addition of diethyl zinc to benzaldehyde. The ligands were tested and it was found that the position of the groups on [2.2] paracyclophane was very important. They concluded this because one of the ligands, shown in Figure 1.15, gave the highest selectivity while its diastereomer gave the lowest. The substituents were in the same relationship to each other but the position was varied causing greatly different results. Once again it was found that both the stereochemistry of the cyclophane and the oxazoline group controlled the enantioselectivity. For the pseudo-ortho diastereomers the selectivity was low. This was thought to be because of the similarity in steric bulk. They concluded that it is important to test every possible isomer when using paracyclophanes as ligands because they can give vastly different results.

Better synthetic routes to enantiomerically pure [2.2]paracyclophane or better separation reactions need to be found. Diethylzinc addition to benzaldehyde is used for $\mathrm{C}-\mathrm{C}$ bond formation, a variety of chiral ligands bearing central, axial and planar chirality have been developed giving good results. Recently disubstituted [2.2] paracyclophane ligands have been reported. Sugiyama et al. optimised diastereomeric monosubstituted [2.2] paracyclophane-based N,O-ligands, which unite 


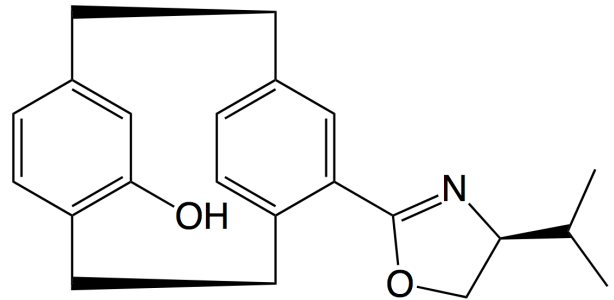

1a $\left(S, R_{p}\right)$

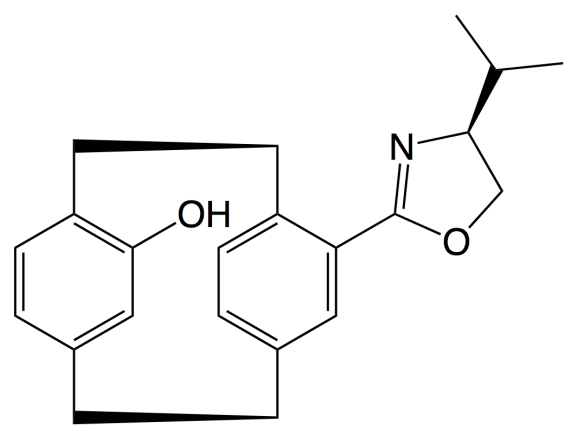

$1 \mathrm{~b}\left(\mathrm{~S}, \mathrm{~S}_{\mathrm{p}}\right)$

FIGURE 1.15: The structure of two diastereomic ligands used for the addition of diethylzinc to benzaldehyde are shown. The 1a-diastereomer showed the lowest selectivity while its diastereomer $\mathbf{1} \mathbf{b}$ showed the highest.

planar and central chirality, for the enantioselective diethyl zinc addition to aldehydes. ${ }^{61}$ This was the first example of monosubstituted [2.2] paracyclophane-based N,O-ligands for this type of reaction. Four ligands were tested, three contained both planar and central chirality while the fourth contained only planar chirality.

The asymmetric addition of diethylzinc to benzaldehyde was carried out in toluene and hexane. The two ligands with relatively small $\mathrm{R}$ groups ( $\mathrm{H}$ and $\mathrm{Me}$ ) had low catalyst efficiencies while the third ligand $(\mathrm{R}=\mathrm{Ph})$ showed excellent selectivity and yields (see Figure 1.16). The diastereomers of this ligand, however, gave completely opposites results ( $91 \%$ and $1 \%$ ee). ${ }^{61}$ The authors hypothesised that the lack of selectivity for the $\left(\mathrm{R}_{\boldsymbol{p}}, \mathrm{S}\right)$-diastereomer was due to more than two active conformations of the catalyst existing in the reaction mixture. Thus both enantiomers of the product were formed at the same rate. This was believed to be because monosubstituted [2.2] paracyclophane-based ligands could adopt a large number of conformations.

The ligand without central chirality gave good selectivity $(41 \%$ ee) but having both central and planar chirality resulted in better selectivity. This was, however, only true when the pair was matched. The effect of planar chirality was also tested by removing the cyclophane and using Bn, Et or Me groups instead. It was found that as the bulkiness of these groups increased the selectivity decreased. A cyclophane, being more bulky than the other, gave better selectivity. The fact that just planar chirality $(41 \%$ ee) and just central chirality $(40 \%$ ee $)$ gave relatively 


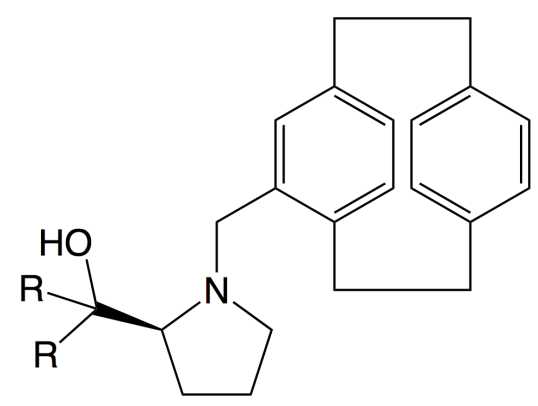

$\mathrm{R}=\mathrm{H}, \mathrm{Me}, \mathrm{Ph}$

FIgURE 1.16: Ligands used for the addition of diethylzinc to benzaldehyde. The small R groups, $\mathrm{H}$ and $\mathrm{Me}$, showed low selectivity while the larger R group, $\mathrm{Ph}$, showed excellent selectivity.

good selectivity has shown the remarkable cooperative effects of the planar and central chiralities. ${ }^{61}$

Asymmetric 1,2-addition reaction of zinc reagents, such as alkyl, alkenyl, aryl, and alkynyl zinc reagents with aldehydes or imines can be controlled using hydroxy[2.2] paracyclophane ketimine ligands. Lauterwasser et al. investigated the non-linear effect, activity, temperature dependence, and kinetics of hydroxy-[2.2] paracyclophane ketamine ligands with the 1,2-addition reaction of diethyl zinc to cyclohexanecarbaldehyde. ${ }^{62}$ Work done previously showed that [2.2]paracyclophane ketimine ligands were highly active and they display matched and mismatched cases. In general it was the paracyclophane backbone that determine the configuration of the product but it was possible to fine-tune the ligand system by altering the side groups. It was found that both enantiomers of the product could be formed and with similar selectivity by using diastereomers that have a different planar-chirality but the same stereogenic centre.

Ligands with a planar chiral backbone are used for hydrogenation of C-C double bonds, reduction of carbonyl and imino groups, and hydroboration. Kreis et al. used 4,5-disubstituted bidentate N,O-ligands in enantioselective organyl zinc addition to aldehydes, imines and 1,4-addition to $\boldsymbol{\alpha}, \boldsymbol{\beta}$-unsaturated aldehydes and ketones. ${ }^{63}$ Synthesis of 4,5-disubstituted [2.2] paracyclophane derivatives was done using different cross-coupling reactions. The derivatives have different heteroatom combinations thus providing potential ligands for asymmetric catalysis. Since previous work on 4,5-disubstituted N,O-ligands was successful variations of these were made. It was thought that by changing the phenolic hydroxy-group to a softer 
amino group it would result in ligands that are better designed for the 1,4-addition to $\boldsymbol{\alpha}, \boldsymbol{\beta}$-unsaturated aldehydes and ketones.

A major problem found in the synthesis of disubstituted [2.2]paracyclophanes is the lack of regioselectivity for the second substitution reaction. For example the electrophilic bromination of 4-bromo[2.2] paracyclophane yields four different stereoisomers. One of the products in their current work was obtained as a single diastereomer. The authors concluded that this was due to the fact that the starting material was fixed in place due to hydrogen-bonding between the phenolic hydroxy-group and the carbonyl-group. When the methyl lithium then tries to attack one side is shielded and only one diastereomer is formed.

Salicyl aldehydes have been used for the synthesis of coumarins and natural products. They are also employed in the synthesis of various imines which are popular ligands since they can be synthesised by condensation with amines, amino alcohols, and diamines. Salicyl aldehydes are therefore important building blocks in organic chemistry because their products can be used in coordination chemistry with a wide range of metal ions. The induction ability of the imine ligand, when used in asymmetric catalysis, therefore depends greatly on the structure of the salicyl aldehyde. The nature of the ligand and thus the induction ability can therefore be optimised by introducing a variety of substituents into either the carbonyl or the amino component.

Zhuravsky et al. synthesised two regioisomeric salicyl aldehyde analogs iso-FHPhPC and pseudo-FHPhPC (see Figure 1.17). ${ }^{64}$ Research showed that bi- and tridentate Schiff base ligands based on the two novel analogs show great selectivity as catalysts in diethyl zinc addition to aldehydes. These show greater chiral induction power than those derived from pure FHPC. An important area for [2.2] paracyclophane research is varying the mutual arrangement of the hydroxy and formyl group of FHPC.

In iso-FHPhPC the imino-moeity was important for controlling the stereochemistry of the product. Matched and mismatched cases were found. Ligands with an additional asymmetric centre provided better selectivity. The tridentate ligand showed medium selectivity which was similar to that of the bidentate ligand. With pseudo-FHPhPC the additional chiral centre which had a positive effect for isoFHPhPC had a negative one here. It was concluded that it was the configuration of the planar chiral PC fragment of the ligand that controlled the configuration of 

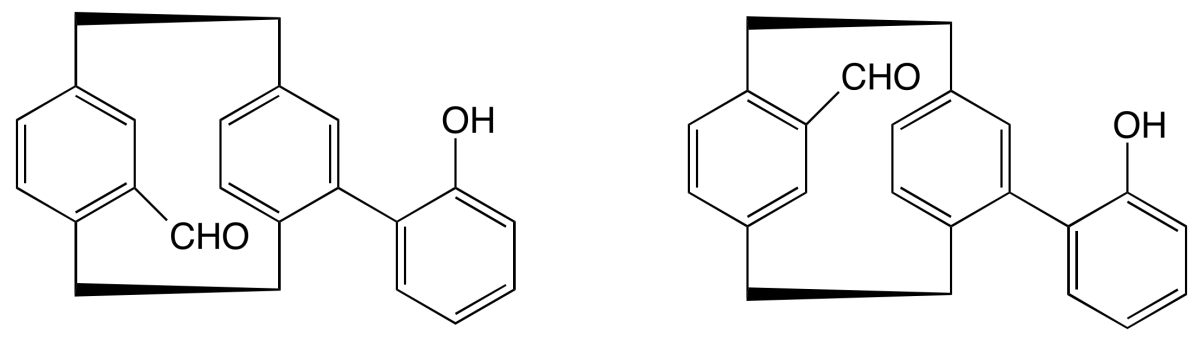

Figure 1.17: The structure of two regioisomeric salicyl aldehyde analogs, isoFHPhPC and pseudo-FHPhPC, are shown.

the product. It was found that the iso- and pseudo-ligands were not as efficient as the FHPC ligand. The best ligand was the diastereomerically pure tridentate iminoalcohol ligand derived from (S)-iso-FHPhPC and (S)-ValOH.

\subsection{Resolution Methods}

During the synthesis of chiral ligands both enantiomers are typically formed. Since only one of these enantiomers will give the desired product these need to be separated before they are incorporated into a reaction. This is also true for chiral auxiliaries. A resolution method is used to try and separate the enantiomers through the formation of diastereomers and many exampes are found throughout the literature. ${ }^{51,65}$ This can either be done using chromatography where the diastereomers are separated using different stationary and mobile phases. Alternatively the diastereomers can be separated through crystallisation as long as their solubility varies enough in the solvent used. Both methods of separation will be explored here. Kinetic resolution ${ }^{50}$ can also be used but this is beyond the scope of this work.

Sugiyama et al. were able to easily separate their diastereomeric monosubstituted [2.2]paracyclophane-based N,O-ligands using silica get column chromatography. ${ }^{61}$ They concluded that the chirality present in the ligands resulted in selectivly forming one product but was also the reason the separation was able to occur. Duan et al. resolved 4-amino-13-bromo[2.2]paracyclophane using (1R)-()-10-camphorsulfonyl chloride, a chiral auxiliary reagent, in the presence of triethylamine. ${ }^{58}$ The diastereomeric amides that formed where then separated using chromatogrpahy on silica gel. The yield of the diastereomers was $47 \%$ and a de of higher than $99 \%$ was obtained with respect to the (-)-diastereomer. ${ }^{58}$ 
Vorontsova et al. also used silica gel column chromatography to separate the diastereomeric Schiff bases formed from S- $\boldsymbol{\alpha}$-PEAM and AHPC. ${ }^{35}$ They unfortunately were not able to get complete separation using just chromatography. Instead a crystallisation in heptane was also used to separate the Schiff bases further. The enantiomers of BHPC were separated by forming its corresponding Schiff bases. Here, a crystalisation was carried out first followed by chromatography.

4-formyl[2.2]paracyclophane is a typical starting point for synthesising other [2.2]paracyclophane derivatives. Consequently it must be enantiomerically pure. One way it has previously been resolved was enzymatically using Saccharomyces cerevisiae. Nowadays the formation of imines is the most common separation method. The disadvantage of forming imines is that they are often unstable on silica. Typically they are resolved by reacting them with chiral reagents which react with the carbonyl groups to give $\mathrm{CN}$ double bonds. These diastereomers are then separated and hydrolysed to return the desired material. Sergeeva et al. proposed three different techniques to separate 4-formyl[2.2]paracyclophane. Using (R)- and (S)2-hydrazino-2-oxo-N-(1-phenylethyl)acetimide was found to be the most effective giving ee's of $99.5 \%$, for the (R)-enantiomer, and $98.7 \%$, for the (S)-enantiomer. ${ }^{66}$

The first method used the resolution method which they had reported earlier using $\boldsymbol{\alpha}$-PEAM. Two crystallisations from hexane gave one diastereomer in a yield of $36 \%$. The enantiomeric purity of $\left(\mathrm{R}_{\boldsymbol{p}}\right)$-4-formyl[2.2]paracyclophane (see Figure 1.18) was determined to be $92 \%$ by HPLC and $98 \%$ by ${ }^{1} \mathrm{H}$ NMR using Mosher's ester. Similar methods gave similar results, 90-92\% ee. The other enantiomer, $\left(\mathrm{S}_{\boldsymbol{p}}\right)$-4-formyl[2.2] paracyclophane (see Figure 1.18), was obtained with an $e e$ of $84 \%$ which was determined by HPLC. To improve the resolution $(\mathrm{S})-\boldsymbol{\alpha}$-(pnitrophenyl)-ethylamine was used instead. Crystallisations in a variety of solvents were unsuccessful and chromatography could not be used because the compounds hydrolysed on $\mathrm{SiO}_{2}$. (S)-2-hydrazino-2-oxo-N-(1-phenylethyl)acetamine was also tried because it had previously been utilised in the resolution of ketones. The same method was applied as mentioned above and $\mathrm{H}_{2} \mathrm{SO}_{4}$ was used for the hydrolys resulting in an ee of $52 \%$ for the (R)-enantiomer. ${ }^{66}$

Zhuravsky et al. resolved racemic bromo-hydroxy[2.2] pracyclophane using a small excess of (1S)-camphanoyl chloride in pyridine. ${ }^{60}$ The diastereomeric esters were then separated by column chromatography on silica gel. The separated diastereomeric esters were then reacted with $\mathrm{KOH}$ in methanol to return the enantiomers. 

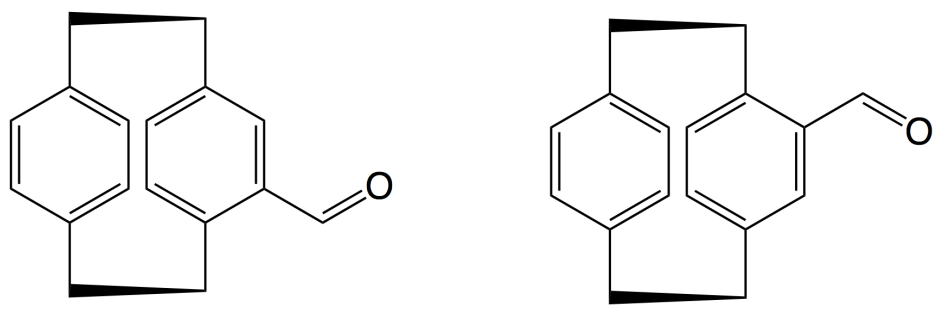

FIgURE 1.18: The structure of $\left(\mathrm{R}_{\boldsymbol{p}}\right.$-4-formyl[2.2]paracyclophane (left) and $\left(\mathrm{S}_{\boldsymbol{p}}\right)$ 4 -formyl[2.2]paracyclophane (right) is shown.

Rozenberg and co-workers separated their two salicyl aldehyde analogs iso-FHPhPC and pseuod-FHPhPC by first forming their corresponding Schiff bases from S- $\boldsymbol{\alpha}$ PEAM. ${ }^{64}$ These were then crystallised in toluene and hexane followed by ethanol. The enantiomers were then recovered using aqueous hydrochloric acid in ethanol. This same method had been used previously. ${ }^{39}$

The resolution of the chiral auxiliary FHPC is particularly interesting due to the fact that the authors proposed a new resolution method soon after their first work was published. The original resolution method by Rozenberg et al. separated the two enantiomers of FHPC by forming their corresponding diastereomeric copper(II) complexes from (S)-valyl-(S)-valine and copper(II) perchlorate (Scheme 1.9) in 2-propanol. The two diastereomers were then separated using silica chromatography. Upon the addition of aqueous $\mathrm{HCl}$ the two enantiomers were recovered with an enantiomeric purity of $94 \%$. The purity of the enantiomers was determined using ${ }^{1} \mathrm{H}$ NMR spectroscopy by using $\left[\mathrm{Eu}(\mathrm{hfc})_{\mathbf{3}}\right]$ as a chiral shift reagent. ${ }^{28}$

The alternative approach by Antonov et al. was reported a year later. ${ }^{39}$ This involved using (R)- $\boldsymbol{\alpha}$-PEAM instead of (S)-valyl-(S)-valine to give an imine. Copper(II) was not used in this reaction. Here (R)- $\boldsymbol{\alpha}$-PEAM was reacted with the racemic FHPC solution to give two diastereomers as shown in Scheme 1.10. The diastereomers were separated capitalising on their differing solubility in hexane. The $\left(\mathrm{R}_{p}, \mathrm{R}\right)$ diastereomer was sparingly soluble in hexane and could be removed from the solution. Upon the addition of aqueous $\mathrm{HCl}$ and methanol $\left(\mathrm{R}_{\boldsymbol{p}}\right)$-FHPC was recovered. The $\left(\mathrm{S}_{\boldsymbol{p}}, \mathrm{R}\right)$-diastereomer was first reacted with aqueous $\mathrm{HCl}$ and methanol to retrieve $\left(\mathrm{S}_{\boldsymbol{p}}\right)$-FHPC followed by the addition of $(\mathrm{S})$ - $\boldsymbol{\alpha}$-PEAM to give the $\left(\mathrm{S}_{\boldsymbol{p}}, \mathrm{S}\right)$-diastereomer which crystallized in hexane. The $\left(\mathrm{S}_{\boldsymbol{p}}, \mathrm{S}\right)$-diastereomer was 


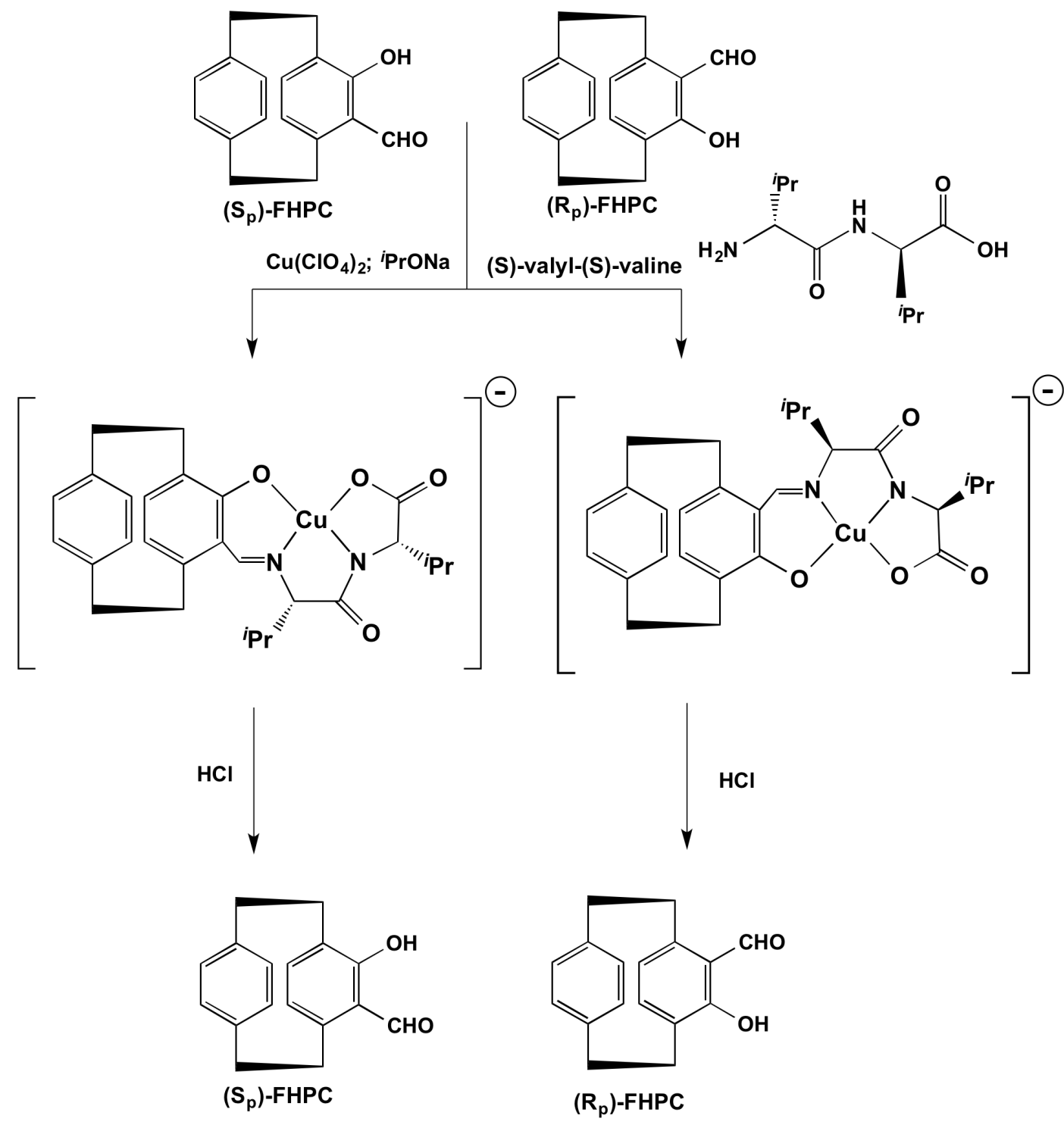

SCHEME 1.9: Reaction scheme showing the resolution of racemic FHPC utilising $\mathrm{Cu}\left(\mathrm{ClO}_{4}\right)$ and $(\mathrm{S})$-valyl-(S)-valine in 2-propanol. The diastereomeric copper complexes were separated using chromatography. $\left(\mathrm{S}_{\boldsymbol{p}}\right)$ - and $\left(\mathrm{R}_{\boldsymbol{p}}\right)$-FHPC were recovered following a hydrolysis reaction using $\mathrm{HCl}$.

then retrieved and following the addition of aqueous $\mathrm{HCl}$ and methanol $\mathrm{S}_{\boldsymbol{p}}$-FHPC was recovered.

The authors believed this resolution method to be simpler because the diastereomers could be separated using crystallisation instead of chromatography. The diastereomers were, however, not completely separated in either of the resolution methods. 
<smiles>O=Cc1c2c(cc3c1CCc1ccc(cc1)CC3)CCC2</smiles><smiles>[Y]CC1CCCCC1</smiles><smiles>C[C@H](N=Cc1c(O)c2c3c(c1CC3)CCC2)c1ccccc1</smiles><smiles>C=C(C)[C@H](C)N=Cc1cc2c(cc1O)CC[C@H]2CC</smiles>

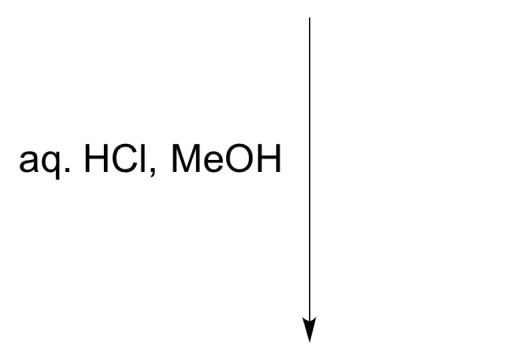
i. aq. $\mathrm{HCl}, \mathrm{MeOH}$<smiles>C[C@H](N)c1ccccc1</smiles>
iii. aq. $\mathrm{HCl}, \mathrm{MeOH}$<smiles>O=Cc1c(O)c2c3c(c1O)CCC3CC2</smiles><smiles></smiles>

SCHEME 1.10: Reaction scheme showing the resolution of racemic FHPC utilising (R)- $\boldsymbol{\alpha}$-PEAM. The diastereomeric imines were then separated by crystallisation. Both enantiomer were recovered through the use of aqueous $\mathrm{HCl}$ in methanol. $\left(\mathrm{S}_{\boldsymbol{p}}\right)$-FHPC was purified further through the use of (S)- $\boldsymbol{\alpha}$-PEAM to give the corresponding diastereomer. $\left(\mathrm{S}_{\boldsymbol{p}}\right)$-FHPC was then recovered using aqueous $\mathrm{HCl}$ in methanol. 


\subsection{Computational Chemistry}

The literature around how to resolve [2.2] paracyclophane derivatives is focused on experimental work. Whereas the majority of theoretical work around [2.2]paracyclophane and its derivatives has mainly centred around mechanistic studies of reactions involving [2.2] paracyclophane as a ligand ${ }^{67}$, structural investigations ${ }^{68}$, absorption propertiesi ${ }^{69-73}$, analysis of electron-density ${ }^{74}$, rotational barriers ${ }^{75}$ and structural investigations ${ }^{76,77}$.

Jiang et al. investigated [2.2] paracyclophane N-Me thiamine chiral reagents in the asymmetric thio-Claisen rearrangement using both B3LYP and M06. ${ }^{78}$ High diastereoselectivities were obtained for this rearrangement thus the authors opted to carry out both experimental and computational work to try and explain this. Planar chiral molecules have not been utilised in this rearrangement due to their instability. [2.2]paracyclophane is, however, chemically stable and will therefore not easily racemize thus making it an ideal planar chiral molecule.

Six different [2.2] paracyclophane derivatives were used as chiral auxiliaries for the asymmetric Claisen rearrangement and resulted in high diastereoselectivites, up to 98\% de. Varying temperature nuclear magnetic resonance was used to determine how rigid the side chain of [2.2] paracyclophane derivatives were. This was done to determine which chiral auxiliary would give the best results relying on the fact that bond rigidity is typically linked with high selectivity. Thus chiral auxiliary 2d was chosen due to its rigid side chain (see Figure 1.19).

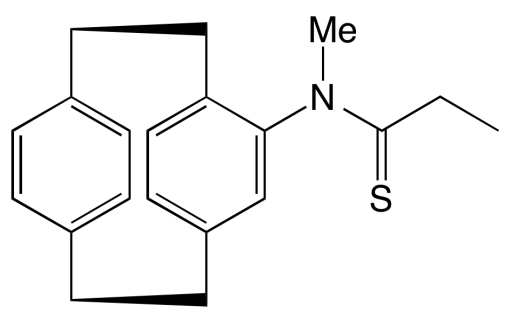

2d

FiguRE 1.19: The structure of the [2.2]paracyclophane derivative used as a chiral auxiliary for the thio-Claisen rearrangement is shown. The side chain of this auxiliary was screened out from five others due to the rigidity of the side chain.

It was determined that the transition state for the thio-Claisen rearrangement would be a concerted process and thus proceed via a chair-like conformation. 
Four pathways were found for this rearrangement due to the starting material having two accessible conformers, transoid and cidsoid, along with the fact that the thiocarbonyl group had two faces where attack could occur, Re and Si. It was found that that Path B was the most feasible, here the rearrangement starts from the cis conformer and proceeds to a product that is more favourable. The energy difference for the transition states for Path B was also larger than for Path A; 2.0 and $0.6 \mathrm{kcal} \mathrm{mol}{ }^{-1}$ respectively. Better diastereoselectivites would therefore be obtained when proceeding through Path B due to the greater energy difference.

The work carried out by Jiang et al. provided a new way of approaching asymmetric synthesis. ${ }^{78}$ The combination of theoretical and experimental work indicated that beginning from a theoretical basis is able to provide exceptional experimental results. In a broader sense asymmetric synthesis can be effectively approached through the use of theoretical predictions. Thus by understanding both the starting material and the reaction in more detail the yield and selectivity can be greatly improved. A step forward for asymmetric synthesis would be to begin to view theoretical work as the foundations of any given investigation. This could greatly aid in the improvement of the experimental process.

Essentially syntheses need to be developed where only one enantiomer is formed or more effective resolution methods need to be developed. The latter is the more realistic starting point. The actual process of the resolution of diastereomers is however not well understood and thus there is no systematic way of improving a resolution method. This is a significant gap in the literature which is difficult to investigate experimentally. Recent experimental procedures such as ultrafast pump-probe X-ray spectroscopy have been used for probing structures close to transition states and this had made significant progress toward observing a transition state experimentally. ${ }^{79}$ Determining a reaction mechanism using computational chemistry can, however, offer a holistic view of the process.

\subsection{Research Aims}

The aim of this research was to investigate the two resolution methods proposed by Rozenberg et al. and Antonov et al. ${ }^{28,39}$ This was achieved by determining a possible reaction profile for each method. In this way diastereomers in the form of intermediates and transition state could be found. This provided insight into why 
a new resolution method was proposed. Since the original resolution method relied on copper and the second did not, the role of the copper ion was determined. Through the in depth analysis and subsequent comparison of the two methods a better understanding of how a resolution method works and how it can be improved is provided. To determine the mechanism of these two resolution methods a computational approach was used since this is the only way it is currently possible to gain an understanding of every step in a reaction. 


\section{Chapter 2}

\section{Methodology}

\subsection{Computational Methods}

All calculations were performed using the Gaussian 09 package. ${ }^{80}$ Optimisations, single point, scan and IRC calculations were performed on a combination of the Victoria University of Wellington School of Chemical and Physical Sciences' (SCPS) Heisenberg cluster and the Victoria University of Wellington Science Faculty HPC facility.

Due to the relative size of the system and the presence of a transition metal ion Densitity Functional Theory (DFT) presented itself as a reasonable method that balanced accuracy and computational effort. The PBE0 hybrid functional was used throughout this investigation because it has been shown to consistently reproduce experimental data for systems containing transition metals and for thermochemistry in general. ${ }^{81} \mathrm{~A}$ triple- $\boldsymbol{\zeta}$ quality basis set, TZVP, was used to ensure an adequate representation of the ground state electron density.

All minima and transition states were optimised. Frequency calculations were performed in order to determine the nature of all stationary points found. To determine the complete reaction profile instrinsic reaction coordinate (IRC) calculations were carried out to match up corresponding minima and thus the reaction pathway can be unambiguously confirmed.

An ultrafine integration grid was used for all calculations. This is a pruned $(99,590)$ gris, with 99 radial shells and 590 angular points per shell, giving 58410 points 
per atom. This was used to try an eliminate small negative frequencies found for some minima.

Relative energies for the reaction pathways were calculated by taking the energy of the minimum or transition state and subtracting the energy of the individual starting materials; FHPC and (S)-valyl-(S)-valine. Relative energies of minima and transition states where water had been removed where calculated by also adding the energy of a water molecule. For the relative energy of the copper complexes the energy of a copper ion was also removed.

A scan of the rotation of the imine in the final product was carried out by altering the dihedral angle by $5^{\circ}$ steps so that the angle was altered by a total of $180^{\circ}$.

Solvent effects for the copper complexes were calculated with the Polarizable Continuum Model using the integral equation formalism variant (IEFPCM). ${ }^{82-102}$ 


\section{Chapter 3}

\section{Results and Discussion (Resolution Method 1)}

The original resolution method proposed by Rozenberg et al. employed $\mathrm{Cu}(\mathrm{II})$ perchlorate. ${ }^{28}$ To determine the mechanism for this resolution method two possible pathways are considered. It is proposed here that the starting materials, FHPC and $(\mathrm{S})$-valyl-(S)-valine, could react to form the imine followed by coordination to the copper ion. Alternatively the starting materials could coordinate to the copper ion first and then react to give the diastereomeric products. The mechanism where the starting material reacts before coordinating will be presented first, i.e. the 'Non-coordinating Pathway', followed by the 'Copper Coordinating Pathway' where coordination occurs before the reaction.

Two different deprotonation steps were found for the second transition state for this resolution method. Both deprotonations were investigated and the results will be presented separately. Firstly the mechanism involving the deprotonation of the amine nitrogen will be introduced; this will henceforth be referred to as the ' $\mathrm{N}$ deprotonation Pathway'. The second mechanism that will be covered involves the deprotonation of the oxygen of the cyclophane hydroxyl group; this will hereafter be known as the 'O-deprotonation Pathway'. 


\subsection{N-deprotonation Reaction Overview}

The first step of the reaction involved the attack of the carbonyl carbon of the cyclophane by the nitrogen from (S)-valyl-(S)-valine (TS1, Figure 3.1). It was found that this occured simultaneously with the attack of one of the hydrogens on the approaching nitrogen by the carbonyl oxygen. The nitrogen-hydrogen bond was consequently broken. This concerted process most likely occured to prevent charge separation i.e. the formation of formally charged species. If this hydrogen migration did not occur simultaneously as the carbon-nitrogen bond formed the nitrogen would be positively charged and, contrariwise, the oxygen negatively charged.

TS1 led to a minimum (Min1, Figure 3.1) where a hydroxyl group was formed along with a bond between the nitrogen and the carbon of the former carbonyl. The hydrogen from the hydroxyl group directly bonded to the cyclophane was facing towards the oxygen of the newly formed hydroxyl group. This minimum was thus stabilised by hydrogen bonding.

The next transition state (TS2, Figure 3.1) led to the ejection of a water molecule. This occured through the formation of a double bond between the nitrogen and the carbon. Consequently the carbon-oxygen bond was broken, which subsequently caused the release of a hydroxyl group. This hydroxyl group deprotonated the nitrogen of the forming imine.

This led to a minimum (Min2, Figure 3.1) where an imine had now been formed and the water molecule interacted with the hydroxyl group on the cyclophane. The minimum was stabilised due to hydrogen bonding between the cyclophane hydroxyl group and the water molecule. The removal of this water molecule led to another minimum (Min3, Figure 3.1) where a rotation had occured to lead to a lower energy minimum. The imine now pointed inward towards the cyclophane hydroxyl group. This allowed for hydrogen bonding to occur between the hydrogen of the hydroxyl group and the nitrogen of the imine.

The imine then coordinated to the copper ion giving diastereomeric copper complexes (Min4, Figure 3.1). Here, the imine was rotated in towards the oxygen on the cyclophane. This allowed for the nitrogen and the oxygen to coordinate to the copper. The nitrogen of the amide and hydroxyl oxygen of the carboxylic acid group were also coordinated to the copper. 


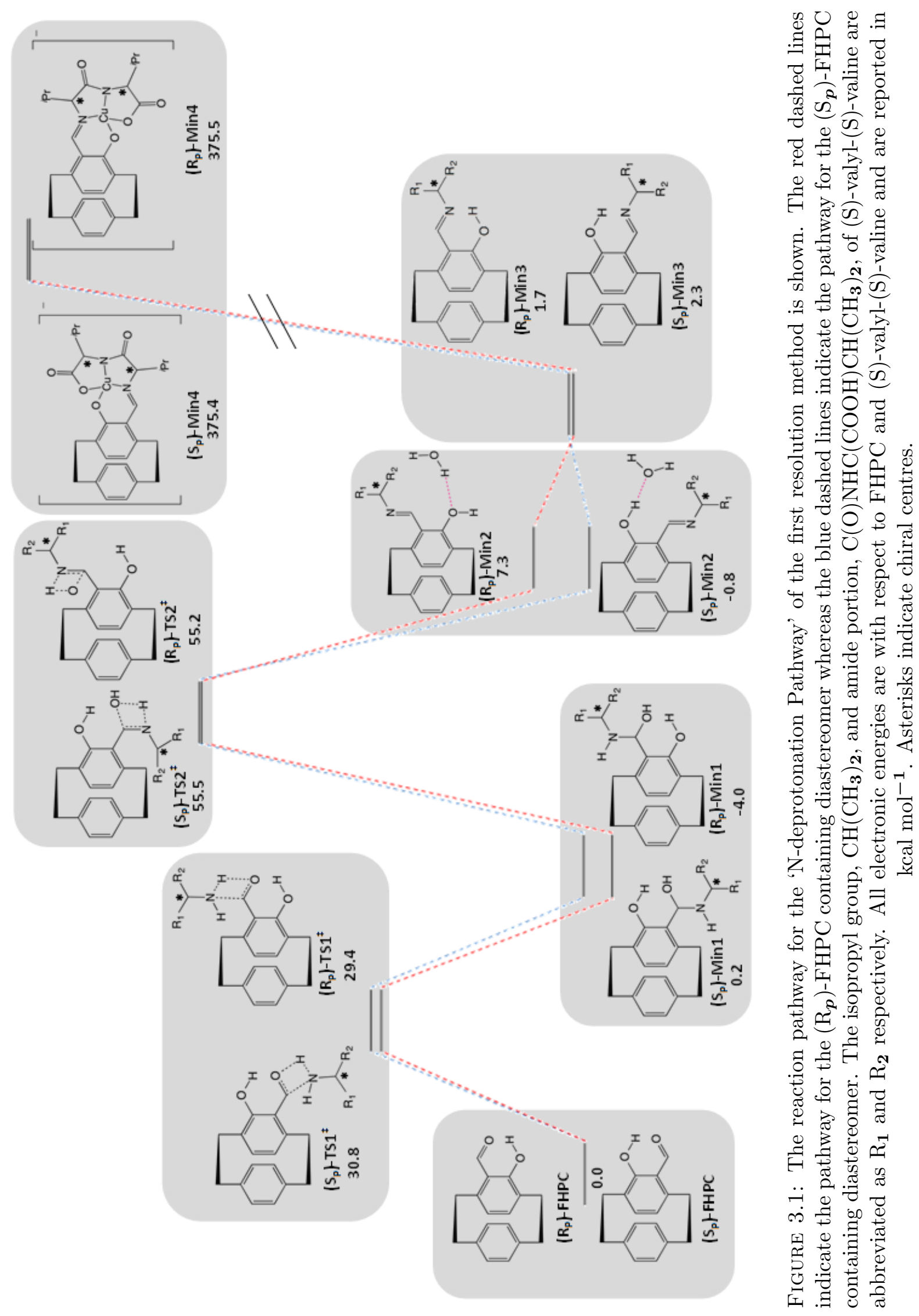




\subsubsection{Transition State 1}

Figure 3.2 shows the 3-dimensional structures of FHPC, the first transition state, TS1, and the first minimum, Min1, for both enantiomers of FHPC. During the first transition state the primary amine of $(\mathrm{S})$-valyl-(S)-valine attacked the cyclophane from below; the plane of the most substituted ring which was not obstructed by the unsubstituted ring. During this attack of the carbonyl carbon, the double bond between this carbon and the oxygen was broken. This occured at the same time as a hydrogen on the nitrogen migrated to the oxygen. Throughout this concerted process no formally charged species were formed.

The resulting minimum only underwent a slight rotation in comparison with TS1. The isopropyl group on (S)-valyl-(S)-valine had not moved. The hydrogen was still oriented towards the cyclophane. This was for steric reasons since the molecule would be lower in energy in this scenario than if a $\mathrm{CH}_{3}$ group were oriented towards the cyclophane.

The other portion of (S)-valyl-(S)-valine had rotated slightly in Min1 compared to the transition state. During TS1 the carbonyl in the amide was oriented away from the carbonyl on the cyclophane. Once again this was to minimise sterics. Throughout the transition state both carbonyls are oriented away from each other since the close proximity of these two oxygens would destabilise the molecule, thus raising the energy. In the minimum the carbonyl in the amide had rotated so it was closer to the newly formed hydroxyl group. This allowed for hydrogen bonding to occur between the hydrogen on the newly formed hydroxyl group and the oxygen in the amide. This interaction stabilised the molecule.

TS1 came about by overcoming an energy barrier of about $30.0 \mathrm{kcal} \mathrm{mol}^{\mathbf{- 1}}$. The resulting minimum, Min1, was lower in energy for the reaction containing $\left(\mathrm{R}_{\boldsymbol{p}}\right)$ FHPC than the reaction containing $\left(\mathrm{S}_{\boldsymbol{p}}\right)$-FHPC; -4.0 and $0.2 \mathrm{kcal} \mathrm{mol}^{\mathbf{- 1}}$ respectively.

\subsubsection{FHPC Rotational Barrier}

The aldehyde functionality on the cyclophane has two faces where the amine was able to attack. The group was free to rotate and ergo the aldehyde could be rotated inwards, which will be referred to as the cis-like conformation, or outwards, the 
a)

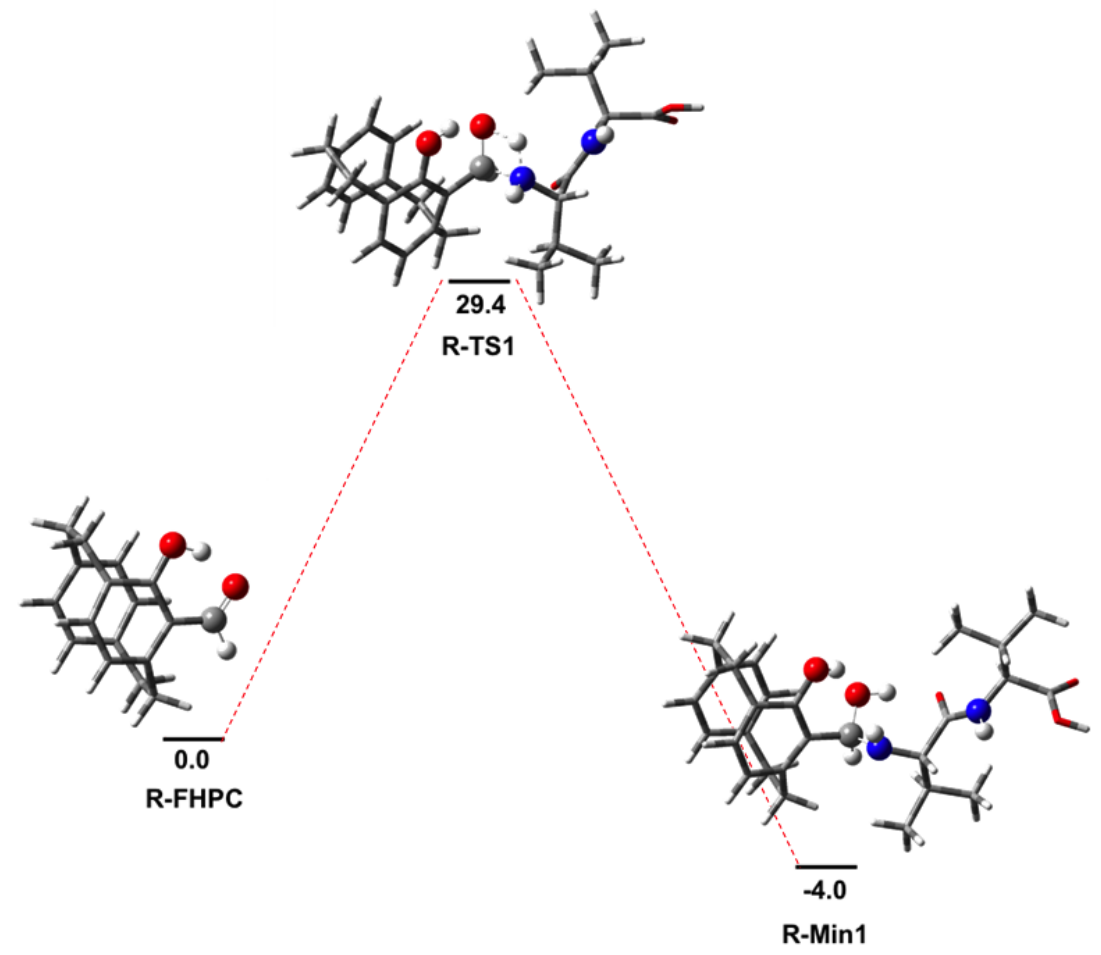

b)
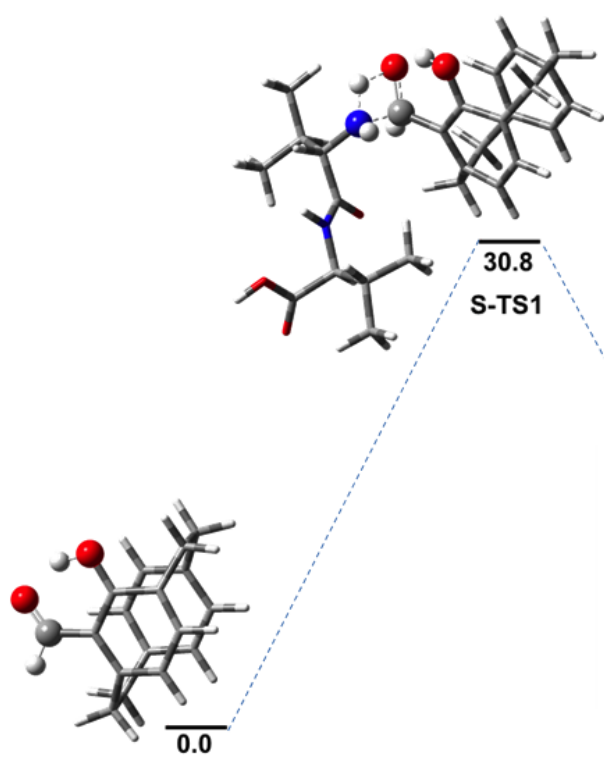

S-FHPC

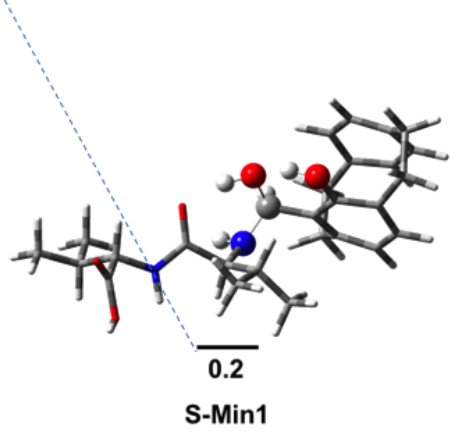

Figure 3.2: The structures of FHPC, TS1, and Min1 for the $\left(\mathrm{R}_{p}\right.$-FHPC containing diastereomer a) and the $\left(\mathrm{S}_{\boldsymbol{p}}\right.$-FHPC containing diastereomer $\left.\mathrm{b}\right)$ are shown. The white, grey, red and blue atoms are $\mathrm{H}, \mathrm{C}, \mathrm{O}$, and $\mathrm{N}$ respectively. The electronic energies are with respect to the starting material, FHPC and (S)-valyl-(S)-valine, and are reported in $\mathrm{kcal} \mathrm{mol}^{-1}$. 
trans-like conformation. The rotational barrier of converting from cis-like to translike, and vice versa, was therefore calculated. Figure 3.3 shows the structures of the cis-like conformer, the trans-like conformer, and the transition state, TS, found when converting between the two.

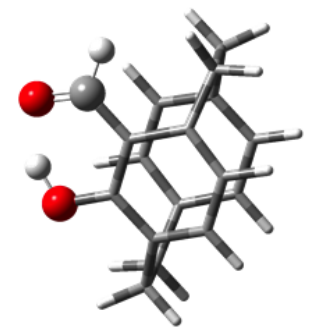

cis-like

conformer

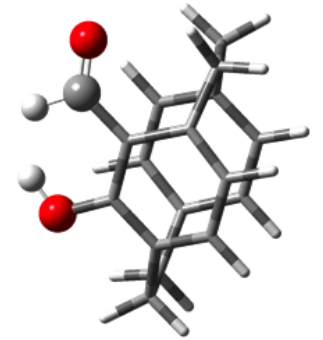

trans-like

conformer

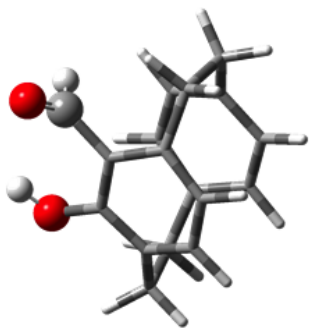

TS

FiguRE 3.3: The structure of the cis-like conformer, the trans-like conformer, and the TS are shown.

By knowing the size of the energy barrier the most prevalent conformer could be determined. Thus attack of (S)-valyl-(S)-valine could be restricted to only occur on one face of the aldehyde as a result of the bulk of the cyclophane shielding the other face of the aldehyde. Since $\left(\mathrm{S}_{\boldsymbol{p}}\right)$-FHPC and $\left(\mathrm{R}_{\boldsymbol{p}}\right)$-FHPC are enantiomers the rotational barrier for this rotation will be identical. It was found that the conversion between the cis-like conformer to the trans-like conformer was $18.3 \mathrm{kcal}$ $\mathrm{mol}^{-1}$ while converting from the trans-like to the cis-like conformer only required about half the energy; $7.2 \mathrm{kcal} \mathrm{mol}^{\mathbf{1}}$ (see Table 3.1).

A plot of the scan is shown in Figure 3.4. The cis-like conformer was found to be the more stable conformation and was therefore used as the starting point for all reaction profiles. This greater stability was most likely due to the fact that a hydrogen bond could be formed between the hydroxyl hydrogen and the carbonyl oxygen (see Figure 3.3). This stabilised the molecule. When the carbonyl was rotated away from the hydroxyl group this was no longer possible thus raising the energy of the molecule by $11.2 \mathrm{kcal} \mathrm{mol}^{-\mathbf{1}}$. During the reaction the carbonyl will therefore be rotated towards the hydroxyl group, and the chiral centre that is formed will have the matching configuration of the cyclophane. This is because the amine attacks from the less hindered side which does not contain the unsubstituted cyclophane ring i.e. below the actual cyclophane. 


\begin{tabular}{lc}
\hline Orientation & Relative Energy $\left(\mathrm{kcal} \mathrm{mol}^{\mathbf{- 1}}\right)$ \\
\hline cis-like & 0.00 \\
trans-like & 11.2 \\
$\mathrm{TS}$ & 18.3 \\
Barrier $_{\text {trans } \rightarrow \text { cis }}$ & 7.1 \\
Barrier $_{\text {cis } \rightarrow \text { trans }}$ & 18.3 \\
\hline
\end{tabular}

TABLE 3.1: The rotational barrier for the conversion from the cis-like conformation, where the carbonyl was facing towards the hydroxyl group, to the trans-like conformation, where the imine was facing away from the hydroxyl group, was calculated for $\left(\mathrm{R}_{\boldsymbol{p}}\right)$-FHPC. Since $\left(\mathrm{S}_{\boldsymbol{p}}\right)$-FHPC and $\left(\mathrm{R}_{\boldsymbol{p}}\right)$-FHPC are enantiomers these values will be exactly identical for $\left(\mathrm{S}_{\boldsymbol{p}}\right)$-FHPC. 'TS' indicates the highest energy point of the rotation. The dihedral angle with respect to the plane created by the two carbons of the cyclophane ring and the plane created by the carbon and oxygen of the carbonyl was varied.

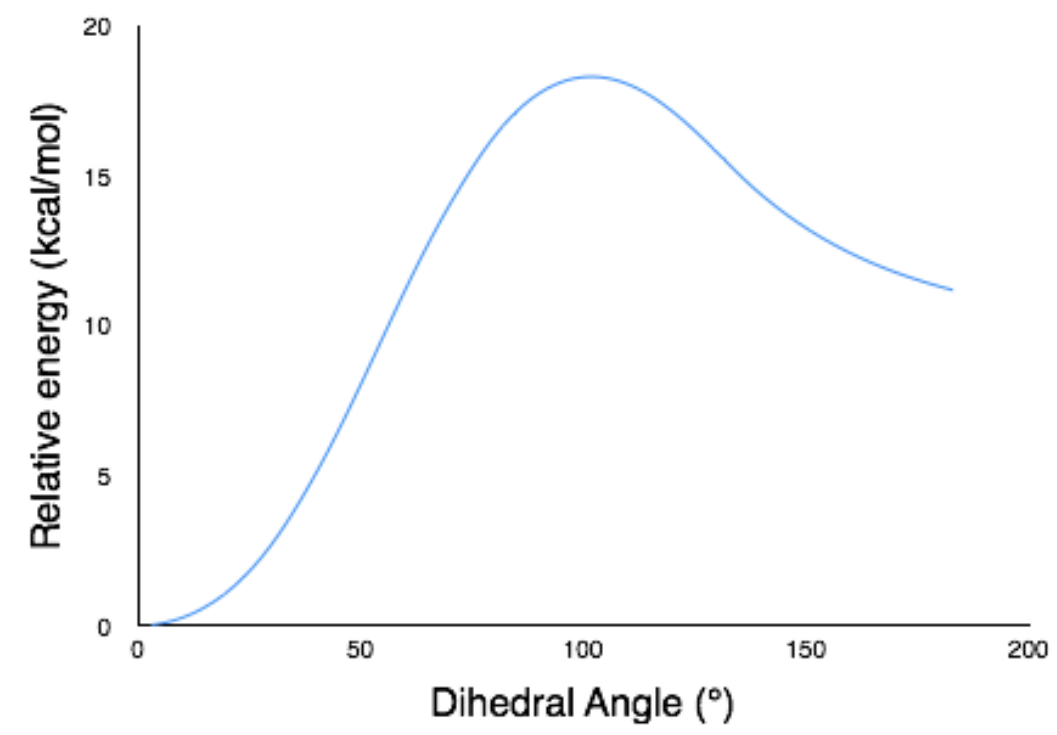

Figure 3.4: A plot of the rotational barrier of converting from cis-like FHPC to trans-like FHPC. The dihedral angle was varied by $5^{\circ}$ over a range of $180^{\circ}$.

\subsubsection{Transition State 2}

During the second transition state, TS2, the newly formed hydroxyl group from the previous minimum, Min1, was ejected through the formation of a water molecule; see Figure 3.5. This occured through the deprotonation of the amine nitrogen which transpired simultaneously as a double bond was formed between 


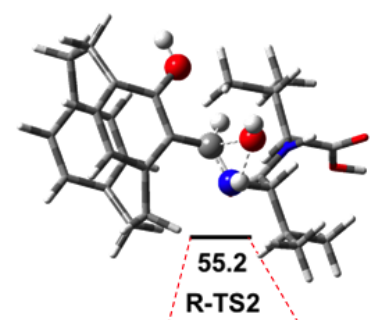

a)
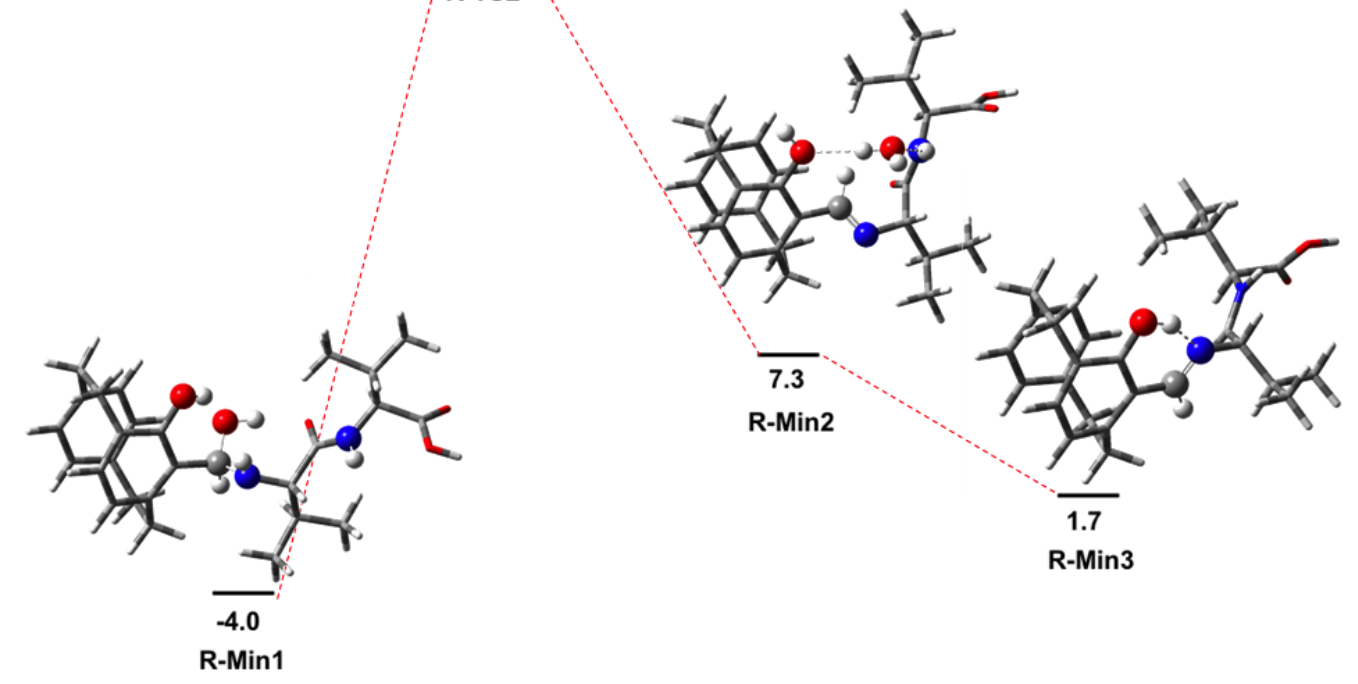

b)

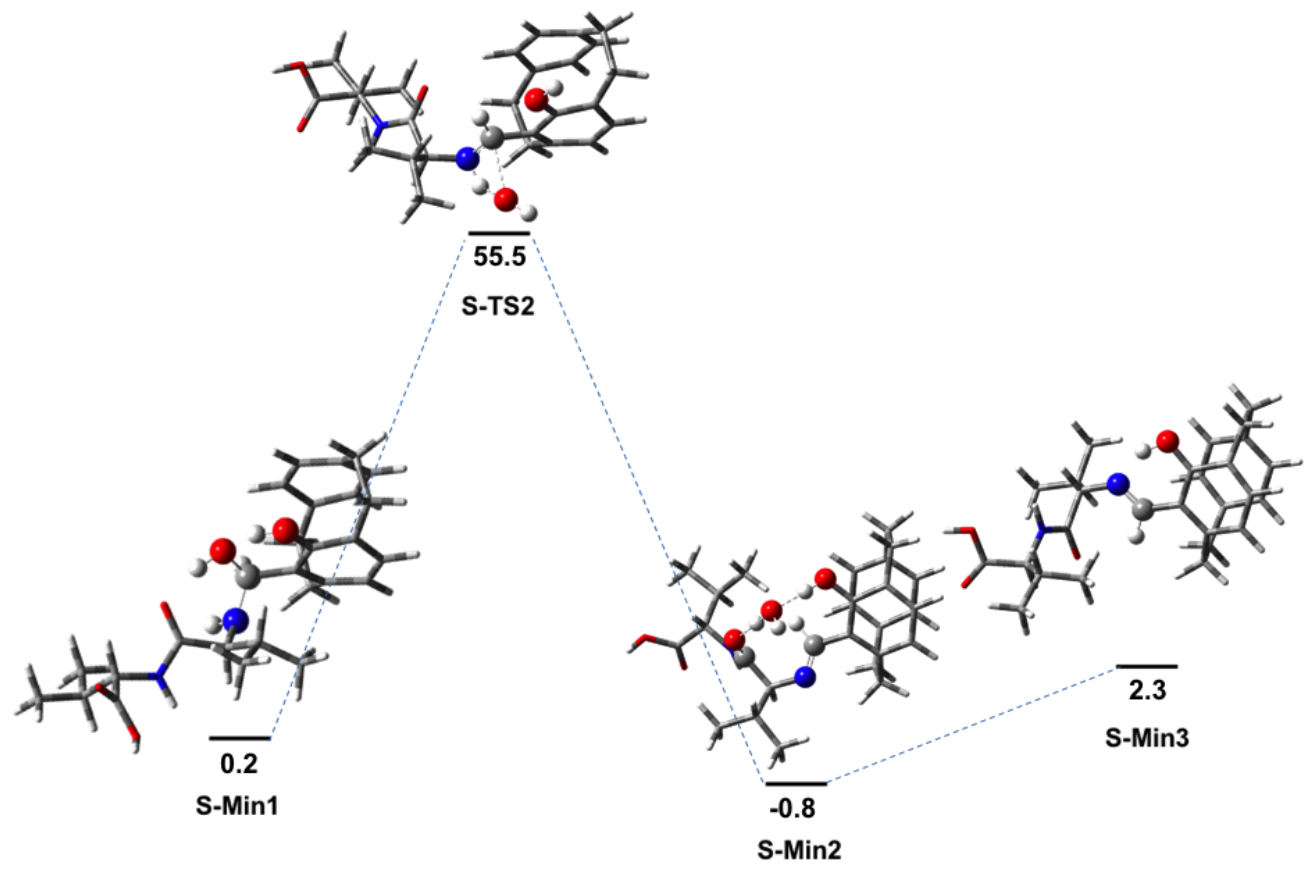

Figure 3.5: The structures of Min1, TS2, Min2, and Min3 for the $\left(\mathrm{R}_{p^{-}}\right.$ FHPC containing diastereomer a) and the ( $\mathrm{S}_{p}$-FHPC containing diastereomer $\mathrm{b}$ ) are shown. The white, grey, red and blue atoms are $\mathrm{H}, \mathrm{C}, \mathrm{O}$, and $\mathrm{N}$ respectively. The electronic energies are with respect to the starting material, FHPC and (S)valyl-(S)-valine, and are reported in $\mathrm{kcal} \mathrm{mol}^{-1}$. 
the nitrogen and carbon. The water molecule was ejected via the bottom of the cyclophane. This required about $55.0 \mathrm{kcal} \mathrm{mol}^{-1}$ to take place.

Figure 3.5 also depicts the structure of Min2 in which the water molecule and imine were now formed. The water molecule hydrogen bonded to both the hydrogen of the cyclophane hydroxyl group and the (S)-valyl-(S)-valine portion of the molecule. The formation of these hydrogen bonds stabilised the molecule to a greater extent for the $\left(\mathrm{S}_{p}\right)$-FHPC diastereomer compared to the $\left(\mathrm{R}_{p}\right)$-FHPC containing diastereomer; -0.8 and $7.3 \mathrm{kcal} \mathrm{mol}^{-1}$ respectively. The orientation of the (S)-valyl-(S)-valine portion of the molecule remained roughly the same.

The water molecule was then removed to give Min3 in which the imine was now oriented in towards the cyclophane hydroxyl group to adopt the cis-like conformation. This allowed for the formation of a hydrogen bond between the hydrogen of the hydroxyl group and the nitrogen of the imine. The $\left(\mathrm{S}_{p}\right)$-FHPC containing diastereomer was now higher in energy than the $\left(\mathrm{R}_{p}\right)$-FHPC containing diastereomer by $0.6 \mathrm{kcal} \mathrm{mol}^{-1}$.

The resulting copper complexes were both much higher in energy than the product; approximately $373.0 \mathrm{kcal} \mathrm{mol}^{-\mathbf{1}}$ higher. The complex containing the $\left(\mathrm{S}_{\boldsymbol{p}}, \mathrm{S}, \mathrm{S}\right)-$ diastereomer was slighly lower in energy by about $0.1 \mathrm{kcal} \mathrm{mol}^{-1}$. Both diastereomeric copper complexes are shown in Figure 3.6.

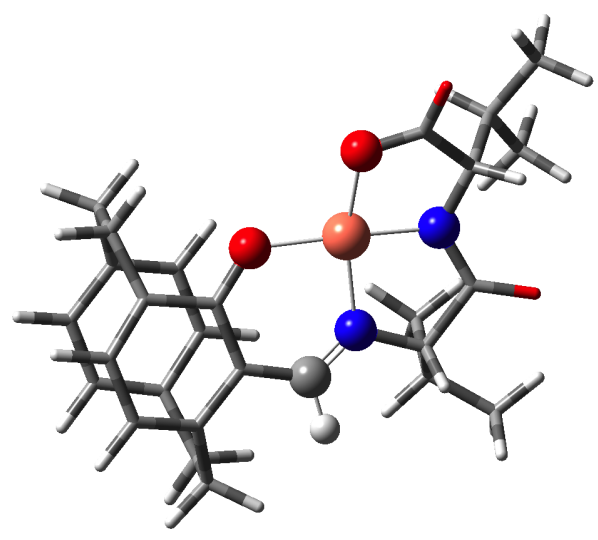

375.4 R-Cu-complex

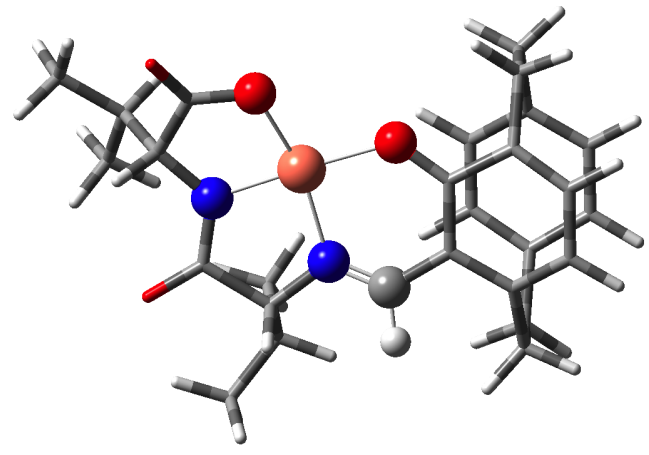

375.5

\section{S-Cu-complex}

FiguRE 3.6: The structure of the diastereomeric copper complexes is shown. The electronic energies are with respect to the starting material, FHPC and (S)-valyl-(S)-valine, and are reported in $\mathrm{kcal} \mathrm{mol}^{-1}$. 


\subsubsection{Rotational Barrier}

During the second transition state there are two possible ways that the forming imine may be positioned. It may be rotated away from the hydroxyl group of the cyclophane, which will be referred to as the trans-like conformer, or it can rotate towards the hydroxyl group during the transition state, which will be referred to as the cis-like conformer. The trans-like conformation is likely to be lower in energy because this places the newly formed hydroxyl group in a better position to deprotonate the hydrogen of the hydroxyl group on the cyclophane. The corresponding minimum, Min3, will however, be higher in energy because hydrogen bonding between the hydrogen on the oxygen and the nitrogen of the imine would no longer possible. To determine whether the rotation of the imine towards the hydroxyl group is feasable the rotational barrier was calculated by scanning the energy change as the dihedral angle was changed.

\begin{tabular}{llc}
\hline Intermediate & Orientation & Relative Energy $\left(\mathrm{kcal} \mathrm{mol}^{\mathbf{- 1}}\right)$ \\
\hline$\left(\mathrm{S}_{\boldsymbol{p}}\right)$-Min3 & cis-like & 0.0 \\
& trans-like & 13.7 \\
& TS & 15.1 \\
& Barrier $_{\text {trans } \rightarrow \text { cis }}$ & 1.4 \\
& Barrier $_{\text {cis } \rightarrow \text { trans }}$ & 15.1 \\
\hline$\left(\mathrm{R}_{\boldsymbol{p}}\right)$-Min3 & cis-like & 0.0 \\
& trans-like & 15.3 \\
& TS & 17.4 \\
& Barrier $_{\text {trans } \rightarrow \text { cis }}$ & 2.1 \\
& Barrier $_{\text {cis } \rightarrow \text { trans }}$ & 17.4 \\
\hline
\end{tabular}

TABLE 3.2: The rotational barrier for converting from the cis-like conformation, imine rotated towards the hydroxyl group, to the trans-like conformation, imine rotated away from the hydroxyl group, was determined for both diastereomers in the reaction; $\left(\mathrm{S}_{\boldsymbol{p}}\right)$-Min3 contains the $\left(\mathrm{S}_{\boldsymbol{p}}\right)$-FHPC enantiomer and $\left(\mathrm{R}_{\boldsymbol{p}}\right)$-Min3 contains the $\left(\mathrm{R}_{\boldsymbol{p}}\right)$-FHPC enantiomer. The dihedral angle with respect to the plane created by the two carbons of the cyclophane ring and the plane created by the carbon and nitrogen of the imine was varied.

Table 3.2 shows the relative energy of the two orientations of the imine, along with the barrier of converting between the two orientations. It can be seen that the cis-like conformation was preferred for both diastereomers. The molecules will therefore adopt the cis-like conformation because this was lower in energy.

A plot of the entire energy change can be seen in Figure 3.7; the relative energies of each step is shown. The lower energy minimum was the cis-like conformation 
of the imine, while the higher energy minimum was the trans-like conformation. The highest energy conformation was about half way through the rotation. Here the imine was below the plane of the substituted ring of the cyclophane.

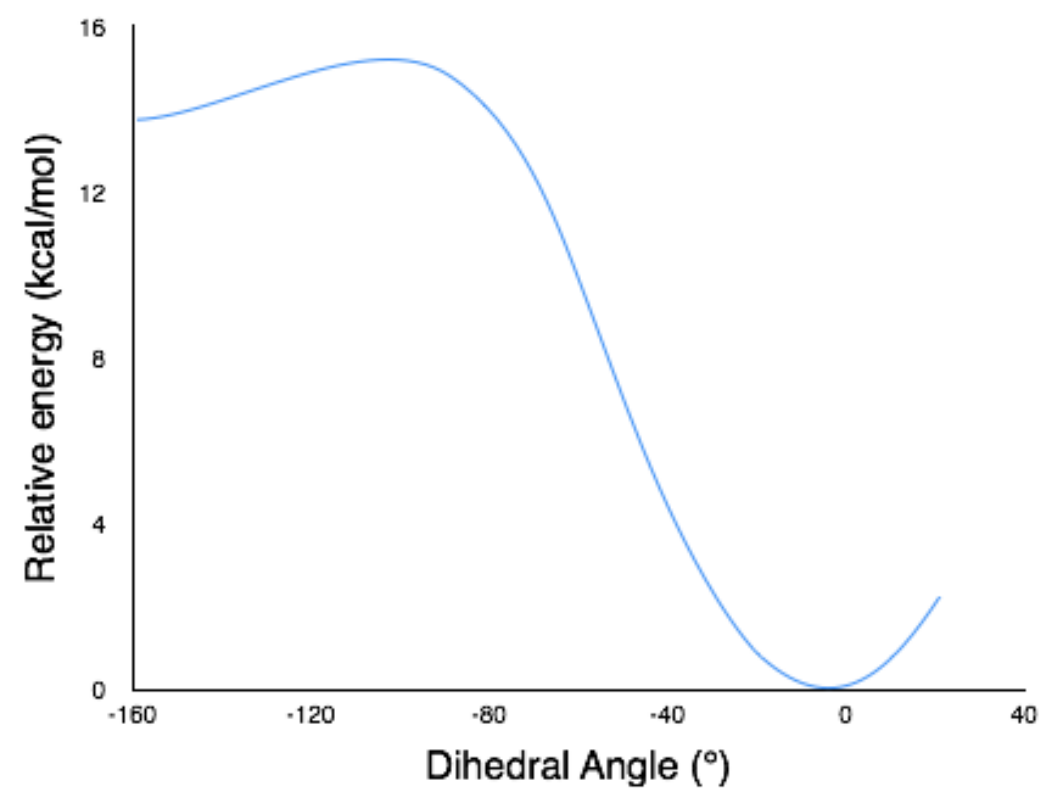

Figure 3.7: The plot of the relative energy for the rotational of the imine in $\left(\mathrm{S}_{\boldsymbol{p}}\right)$-Min3 is shown. The dihedral angel was altered by $5^{\circ}$ for 36 steps, thus covering $180^{\circ}$. The lowest energy minimum was found for when the imine is rotated in towards the hydroxyl group on the cyclophane thus allowing for hydrogen bonding. The higher energy minimum was when the imine was rotated away. The highest energy point was about halfway through the rotation.

The barrier for converting from the less stable trans-like conformer was 1.4 and 2.1 kcal mol ${ }^{-1}$ for the $\left(\mathrm{S}_{\boldsymbol{p}}\right)$-FHPC and $\left(\mathrm{R}_{\boldsymbol{p}}\right)$-FHPC containing diastereomer respectively. Since these barriers are relatively low in energy the cis-like conformation is easily accessible. The barrier for converting from the cis-like conformation to the trans-like conformation for the $\left(\mathrm{S}_{\boldsymbol{p}}\right)$-FHPC containing diastereomer was found to be $15.1 \mathrm{kcal} \mathrm{mol}^{-\mathbf{1}}$. Since this barrier was fairly large the imine would not rotate and convert back to the trans-like conformation during the reaction. The barrier for the conversion between the cis-like to the trans-like conformer for the $\left(\mathrm{R}_{\boldsymbol{p}}, \mathrm{R}, \mathrm{S}, \mathrm{S}\right)$ diastereomer was $17.4 \mathrm{kcal} \mathrm{mol}^{\mathbf{- 1}}$.

The structures for the cis-like and trans-like conformers are showing in Figure 3.8 along with the structure of the transition state, TS. 


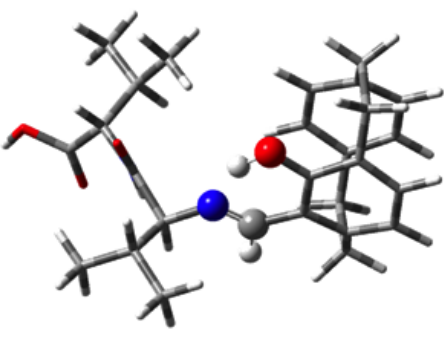

(S)-cis-like conformer

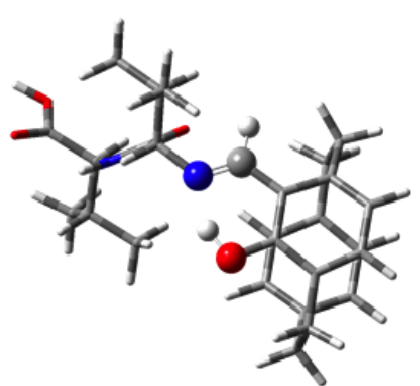

(R)-cis-like conformer

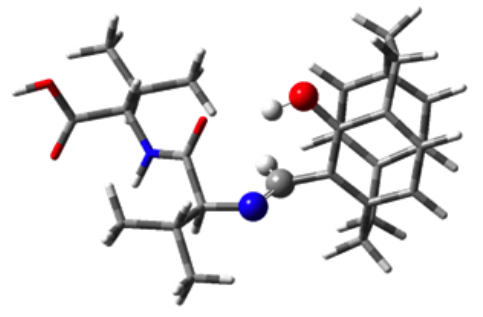

(S)-trans-like conformer

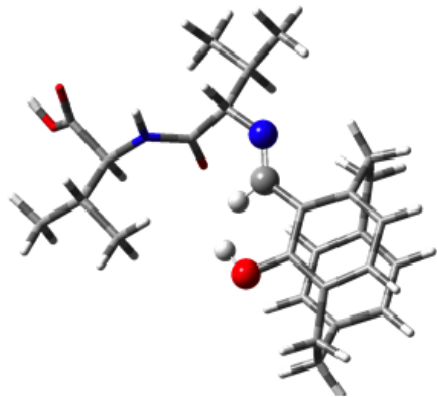

(R)-trans-like conformer

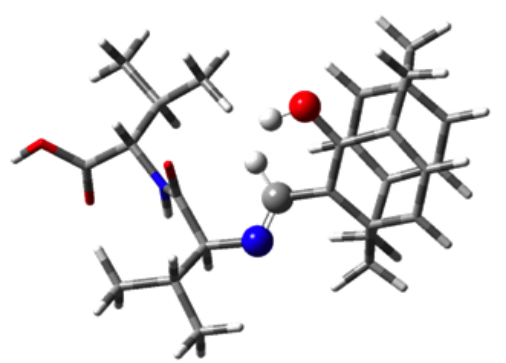

(S)-TS

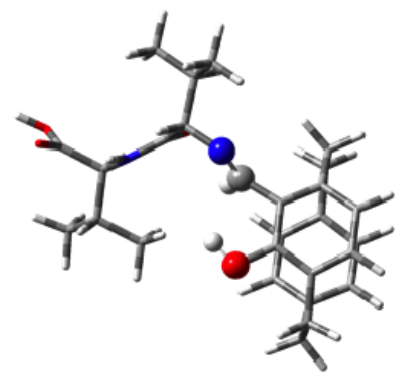

(R)-TS

FiguRE 3.8: The structure of the cis-like conformer, the trans-like conformer and the transition state, TS, are shown.

\subsection{O-deprotonation Reaction Overview}

The 'O-deprotonation Pathway' for this resolution method proceeded through the identical first transition state (TS1, Figure 3.9) giving the identical minimum (Min1, Figure 3.9) and final products (Min4 and Min5, Figure 3.9). The pathway deviated at the second transition state; deprotantion and removal of water. In this 'O-deprotonation Pathway' the hydroxide that was removed did not deprotonate the nitrogen, which would have lead to the product, but instead deprotonated the hydroxide of the cyclophane. Once more this was a concerted step where the hydroxyl group of the cyclophane was deprotonated as the newly formed hydroxyl group was ejected by the formation of a double bond between the carbon and the nitrogen.

This led to the second minimum (Min2, Figure 3.9) where the newly formed imine was either oriented away from the deprotonated hydroxyl group on the 

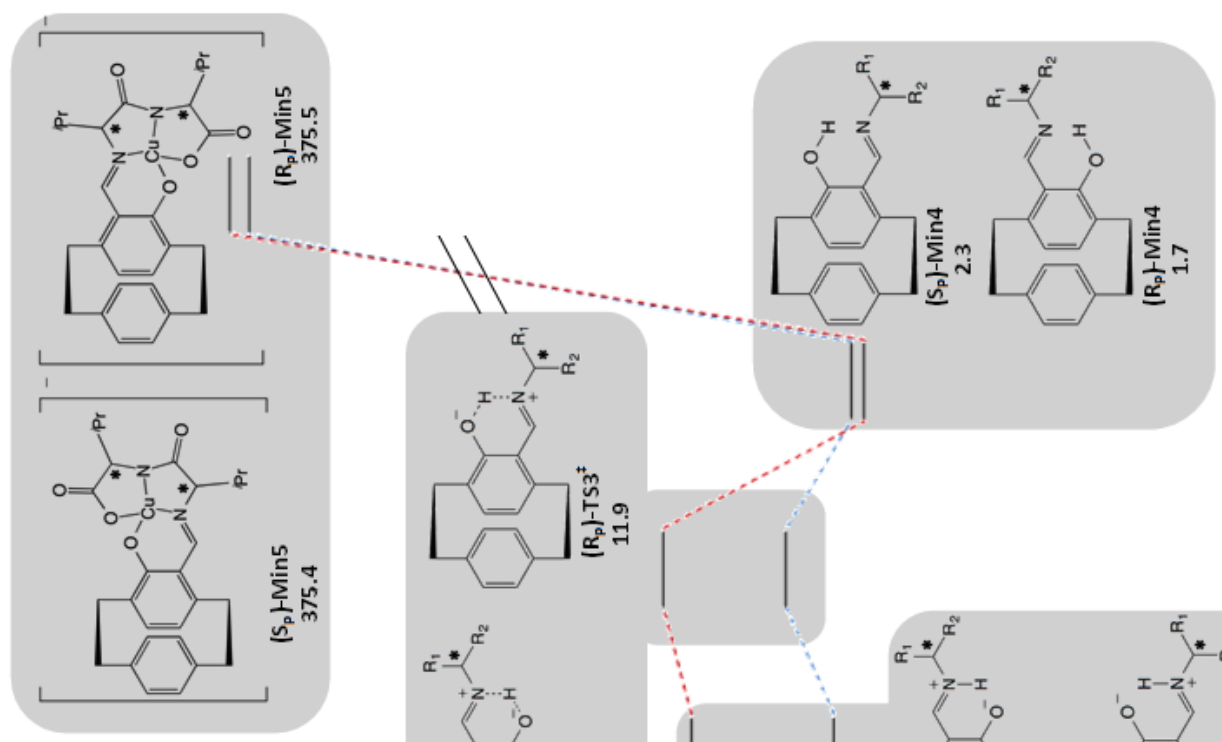

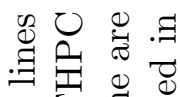

昰

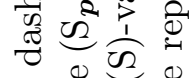

过 $\frac{1}{1}$

워

E. 家的

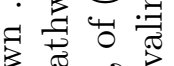

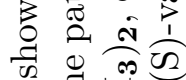

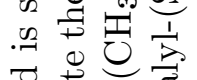

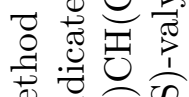

票贾

:

苛

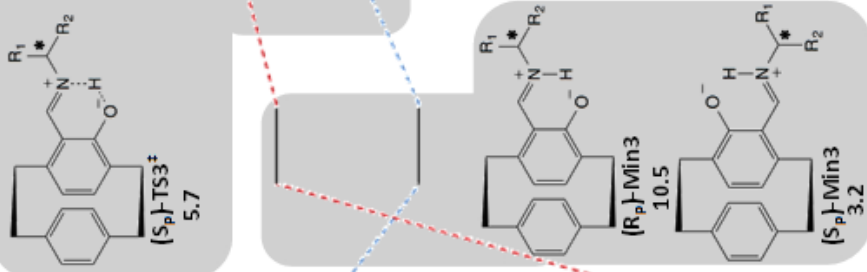

总党応

wo 0

ज声

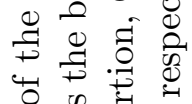

o क

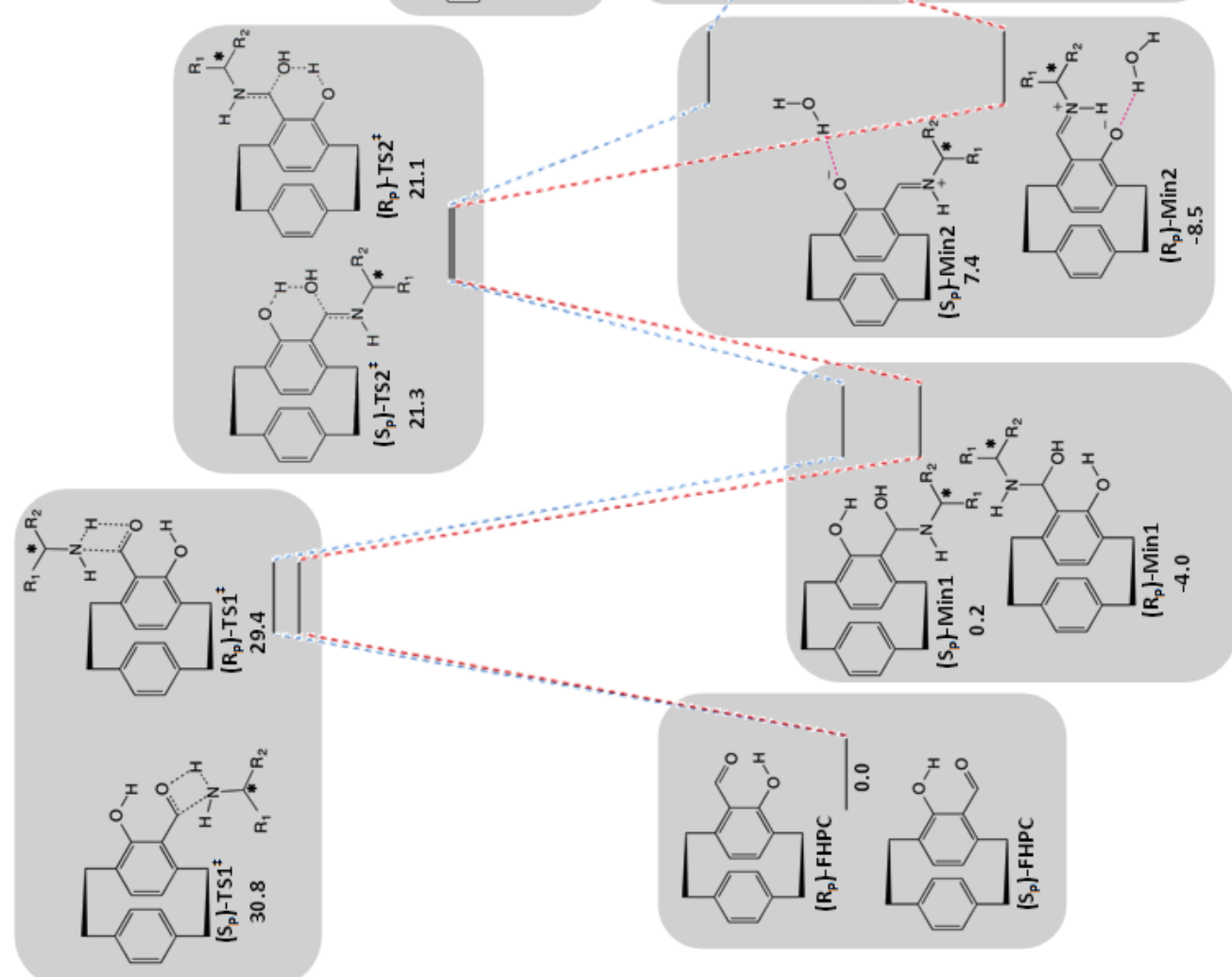

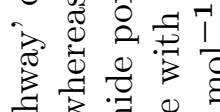

考范

A

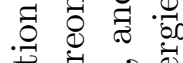

节 峁

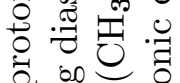

岂.

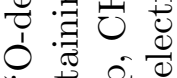

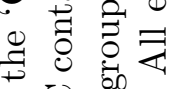

을

考茫总

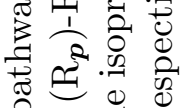

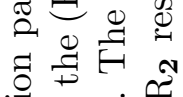

苞泀它

¿

空密

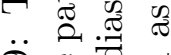

के \& 00

के

될

总. 曾 
cyclophane or towards it. The hydrogen that was removed from the hydroxyl group on the cyclophane hydrogen bonded with this formally negatively charged oxygen. Removal of the water molecule led to another minimum (Min3, Figure 3.9). The imine was now oriented towards the negatively charged oxygen which allowed for hydrogen bonding between this oxygen and the imine hydrogen to occur.

The final transition state (TS3, Figure 3.9) involved a proton transfer between the hydrogen of the nitrogen to the negatively charged oxygen. The rotation of the imine between the second and third minimum allowed for this step to proceed with greater ease. This then led to the final product (Min4, Figure 3.9) where the hydroxyl group on the cyclophane was reformed. The imine was still oriented towards this hydroxyl group which allowed for the formation of a hydrogen bond between the hydroxyl hydrogen and the imine nitrogen. This molecule then coordinated to copper(II) as with the 'N-deprotonation Pathway' above to give the final product (Min5, Figure 3.9).

\subsubsection{Transition State 2}

During the second transition state the newly formed hydroxyl group was removed by the formation of water. Figure 3.10 depicts the structure of the second transition state, TS2, the second minimum, Min2, and the third minimum, Min3. It can be seen that in TS2 a water molecule was formed by deprotonation at the cyclophane hydroxyl group. This occured as the carbon-oxygen bond was broken and a double bond was formed between the carbon and the nitrogen. The water molecule was able to leave either from the side that contained the cyclophane or from the other. This transition state overcame a barrier of around $22.0 \mathrm{kcal} \mathrm{mol}^{-1}$.

Figure 3.10 also depicts the structure of Min2. Here the dashed lines indicate hydrogen bonds. The (S)-valyl-(S)-valine portion of the molecule was oriented so that no bulk was around the water molecule. The water molecule was still in close proximity to the oxygen that was deprotonated thus allowing for a hydrogen bond between them. This formally negatively charged oxygen also interacted with the hydrogen of the newly formed imine. The oxygen of the water molecule formed a hydrogen bond with the hydrogen of the amide nitrogen. These hydrogen bonds stabilised the molecule. The orientation of the other groups are, however, unfavourable due to their bulk and being oriented more towards the cyclophane 
than usual. The energy of the minimum containing the $\left(\mathrm{R}_{\boldsymbol{p}}\right)$-FHPC cyclophane was much lower in energy compared to the minimum with the $\mathrm{S}_{\boldsymbol{p}}$ enantiomer; -8.5 and $7.4 \mathrm{kcal} \mathrm{mol}^{\mathbf{1}}$ respectively.

Min3 (Figure 3.10) no longer contained a water molecule. The (S)-valyl-(S)valine portion was oriented so that the amide nitrogen now interacted with the formally negatively charged oxygen. This oxygen still interacted with the hydrogen on the imine nitrogen. The energy of the minimum containing the $\left(\mathrm{R}_{p}\right)$-FHPC enantiomer was now higher in energy, $10.5 \mathrm{kcal} \mathrm{mol}^{-1}$, than that of the $\left(\mathrm{S}_{\boldsymbol{p}}\right)$-FHPC containing diastereomer, $3.2 \mathrm{kcal} \mathrm{mol}^{-1}$.

\subsubsection{Rotational Barrier}

The rotational barrier for the 'O-deprotonation Pathway' was also calculated since the energies would differ due to the nitrogen being formally postively charged. Table 3.3 shows the energy of the trans-like conformer, the cis-like conformer and the transition state separating the conformers for the $\left(\mathrm{S}_{\boldsymbol{p}}\right)$-FHPC containing diastereomer, along with the barrier for converting between the two. A scan of only the $\left(\mathrm{S}_{\boldsymbol{p}}\right)$-FHPC containing diastereomer was carried out since the energy of the diastereomer for the previous pathway only differed slightly. Similar energy differences would therefore also be expected here.

For TS3 to occur, where the hydrogen on the nitrogen transfers to the oxygen, the nitrogen in the imine must be rotated towards the formally negatively charged oxygen. This decreases the distance the hydrogen has to migrate and the close proximity in the cis-like conformer compared to the trans-like conformer would result in a lower energy third transition state.

The cis-like conformer was lower in energy than the trans-like conformer. The barrier for converting from the trans-like to the cis-like conformer was found to be $27.6 \mathrm{kcal} \mathrm{mol}^{-1}$, nearly twice that for the rotation in the 'N-deprotonation Pathway'. Converting from the favourbale cis-like conformer to the trans-like conformer required $39.8 \mathrm{kcal} \mathrm{mol}^{\mathbf{- 1}}$. This was nearly 30 times larger than for the 'N-deprotonation Pathway'. The majority of the molecules would therefore be in the cis-like conformation since it is the more stable conformer. Any molecules in the trans-like conformation would require energy to convert from the trans- like to the cis-like conformer. Experimentally this reaction was was refluxed therefore 
a)

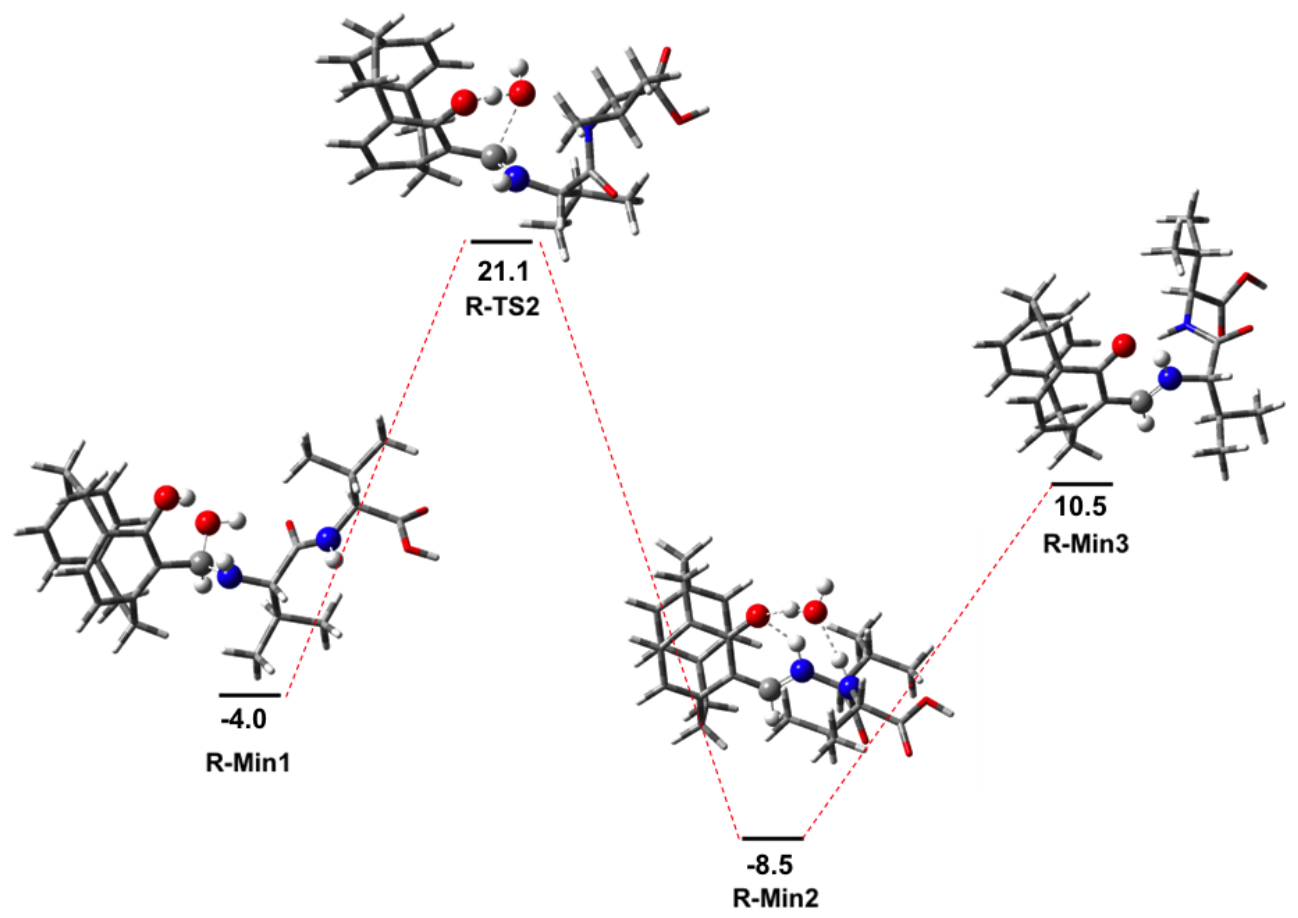

b)

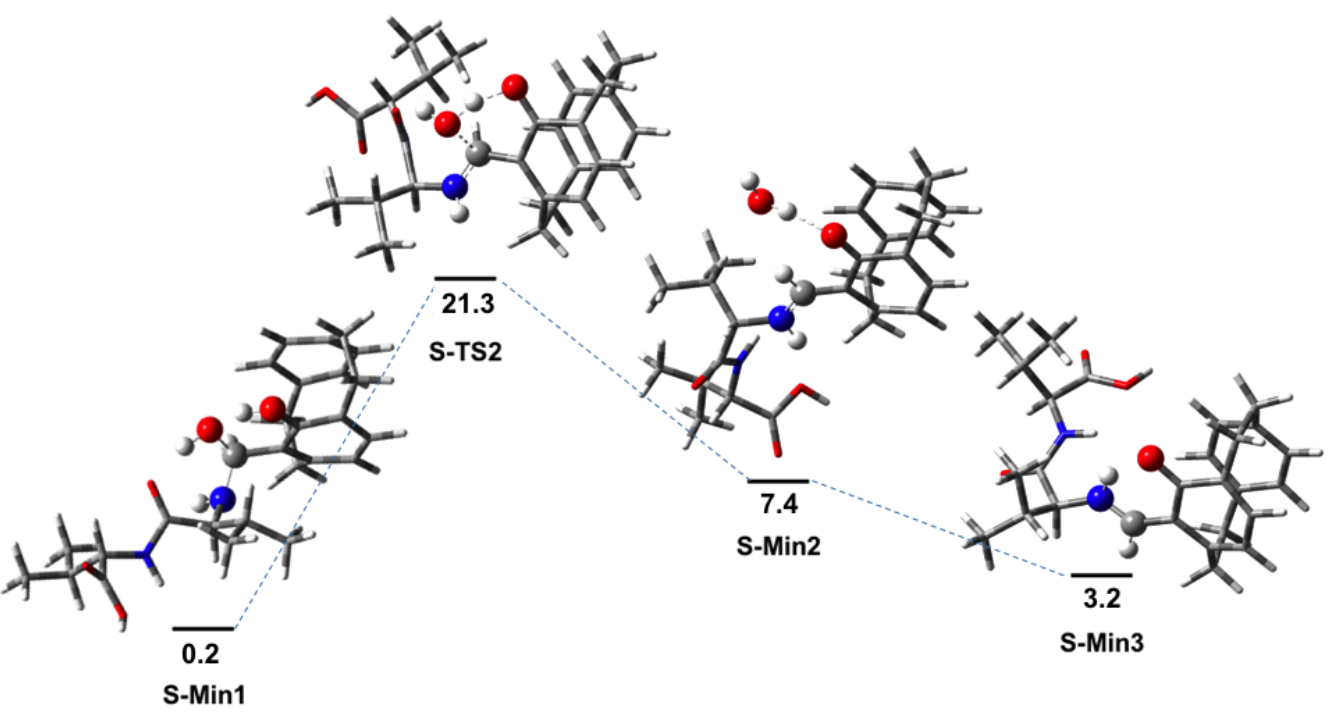

Figure 3.10: The structures of Min1, TS2, Min2 and Min3 for the $\left(\mathrm{R}_{p^{-}}\right.$ FHPC containing diastereomer a) and the ( $\mathrm{S}_{\boldsymbol{p}}$-FHPC containing diastereomer $\mathrm{b}$ ) are shown. The white, grey, red and blue atoms are $\mathrm{H}, \mathrm{C}, \mathrm{O}$, and $\mathrm{N}$ respectively. The electronic energies are with respect to the starting material, FHPC and (S)valyl-(S)-valine, and are reported in $\mathrm{kcal} \mathrm{mol}^{-1}$. 


\begin{tabular}{llc}
\hline Intermediate & Orientation & Relative Energy $\left(\mathrm{kcal} \mathrm{mol}^{\mathbf{- 1}}\right)$ \\
\hline $\mathrm{S}_{\boldsymbol{p}}$ Min3 & cis-like & 0.0 \\
& trans-like & 12.2 \\
& $\mathrm{TS}$ & 39.8 \\
& Barrier $_{\text {trans } \rightarrow \text { cis }}$ & 27.6 \\
& Barrier $_{\text {cis } \rightarrow \text { trans }}$ & 39.8 \\
\hline
\end{tabular}

TABLE 3.3: The rotational barrier for converting from the cis-like conformation, imine rotated towards the hydroxyl group, to the trans-like conformation, imine rotated away from the hydroxyl group, was determined for the $\left(\mathrm{S}_{\boldsymbol{p}}\right)$-FHPC containing diastereomer. The dihedral angle with respect to the plane created by the two carbons of the cyclophane ring and the plane created by the carbon and nitrogen of the imine was varied.

the cis-like conformer becomes accesible even though the barrier associated with it is large. Conversion from the cis-like to the trans-like conformer would also be accessible but since the subsequent transition state, TS3, required the hydrogen of the imine to be in close proximity to the formally negatively charged oxygen the cis-like conformer will be preferred.

\subsubsection{Transition State 3}

During the third transition state the hydrogen on the imine nitrogen migrated to the formally negatively charged oxygen to reform the hydroxyl group on the cyclophane. During this transition state the orientation of the $(\mathrm{S})$-valyl-(S)-valine remains identical as seen in Figure 3.11. This was most likely due to the hydrogen migrating over a very short distance. It was travelling in a straight line because of the rotation that had occured to give Min3. Since this was such a short distance, that only required direct travel, the chain of the molecule did not need to reorganise itself.

In the final product, Min4, the (S)-valyl-(S)-valine chain had rotated. The carbonyl in the amide was now rotated away, further towards the other end of the cyclophane. The lower isopropyl group was now oriented so that the hydrogen on the first carbon was further towards the cyclophane. The upper isopropyl group was oriented so that one of the $\mathrm{CH}_{3}$ groups was pointing more towards the cyclophane. The only hydrogen bonding possible in Min4 was between the hydrogen of the oxygen and the nitrogen of the imine. 
The final transition state, TS3, was the lowest energy barrier in this reaction; about $2.0 \mathrm{kcal} \mathrm{mol}^{-1}$. For the corresponding minimum, Min4, the reaction with the $\left(\mathrm{R}_{p}\right)$-FHPC containing diastereomer was once again lower in energy than that with the $\left(\mathrm{S}_{\boldsymbol{p}}\right)$-FHPC containing diastereomer; 1.7 and $2.3 \mathrm{kcal} \mathrm{mol}^{-1}$ respectively.

a)

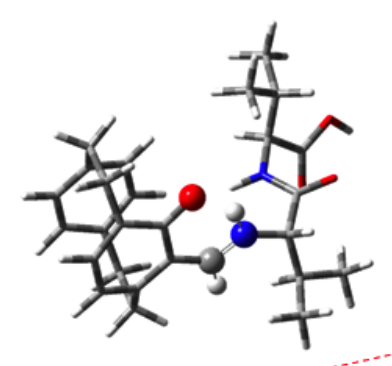

$\overline{10.5}$

R-Min3

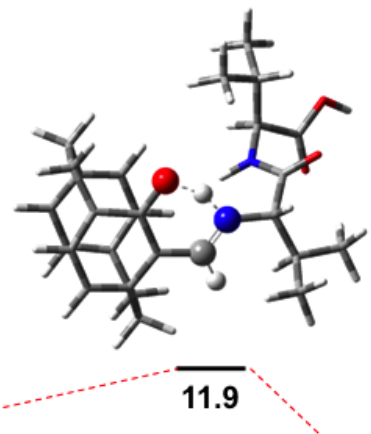

R-TS3

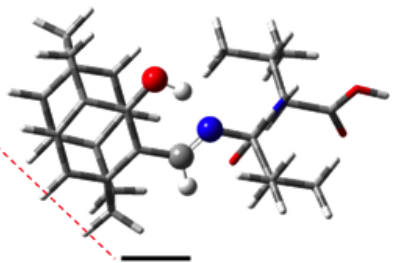

1.7

b)

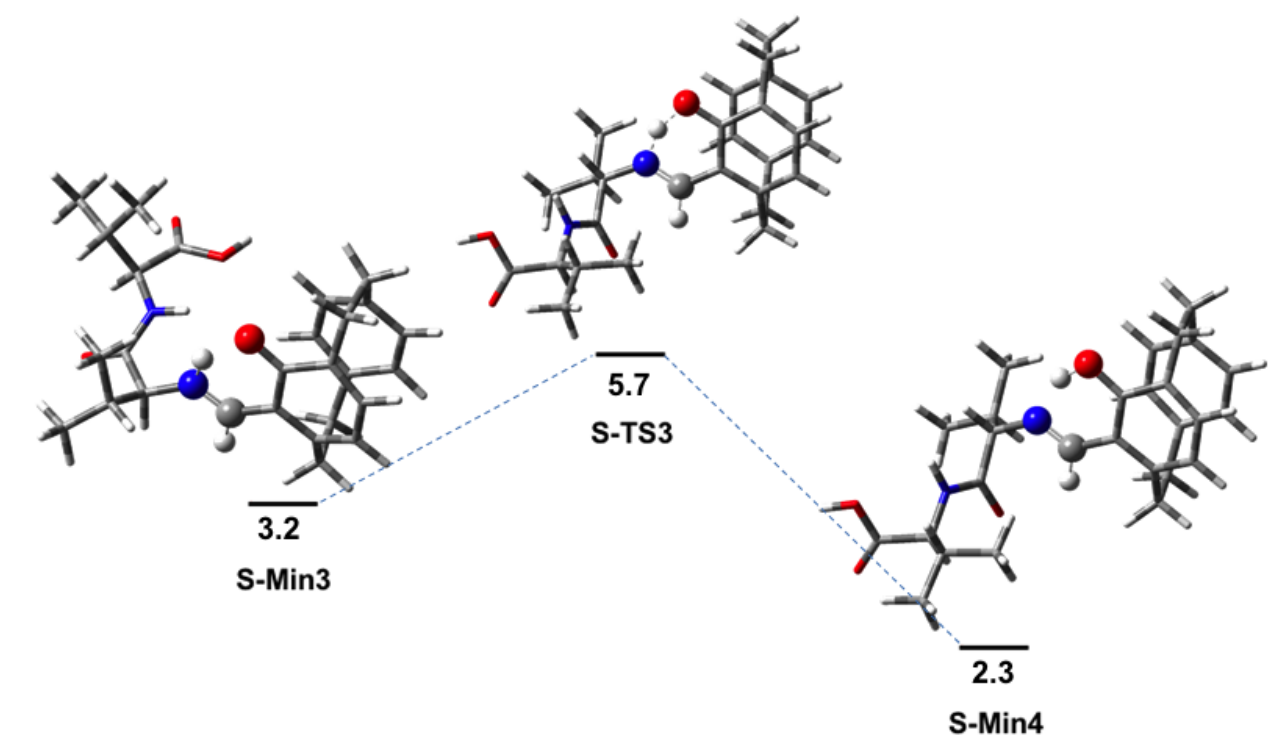

Figure 3.11: The structures of Min3, TS3, and Min4 for the $\left(\mathrm{R}_{p}\right.$-FHPC containing diastereomer a) and the $\left(\mathrm{S}_{\boldsymbol{p}}\right.$-FHPC containing diastereomer $\left.\mathrm{b}\right)$ are shown. The white, grey, red and blue atoms are $\mathrm{H}, \mathrm{C}, \mathrm{O}$, and $\mathrm{N}$ respectively. The electronic energies are with respect to the starting material, FHPC and (S)-valyl-(S)-valine, and are reported in $\mathrm{kcal} \mathrm{mol}^{-\mathbf{1}}$. 


\subsection{Copper Coordinating Pathway}

Due to time constraints this pathway was not explored in full. The first transition state was found which allowed for a comparison with the 'Non-Coordinating Pathway' mentioned above. During the transition state the nitrogen attacked the carbonyl carbon as a hydrogen on the nitrogen migrated to the oxygen of the carbonyl. This transition state was identical for both the 'Coordinating' and 'NonCoordinating Pathway'. The way the molecule was oriented here was different, however, due to the fact that it was coordinated to the copper ion.

The copper ion was situated below the plane of the most substituted ring of the cyclophane, which was not obstructed by the unsubstituted ring. It was coordinated to one of the oxygens of the carboxyilc acid group, the oxygen of the cyclophane, and the nitrogen of the amide. During the transition state the distance between the copper ion and the nitrogen attacking the carbon decreased. This suggested that in the corresponding minimum the copper ion would also be coordinated to this nitrogen.

\begin{tabular}{lc}
\hline Pathway & Relative Energy $\left(\mathrm{kcal} \mathrm{mol}^{\mathbf{- 1}}\right)$ \\
\hline Copper Coordinating & 428.0 \\
Non-Coordinating & 29.4 \\
\hline
\end{tabular}

TABle 3.4: The electronic energies for the first transition state, TS1, for the $\left(\mathrm{R}_{\boldsymbol{p}}\right)$-FHPC containing diastereomer for the 'Copper Coordinating Pathway' and the 'Non-Coordinating Pathway' are reported.

Table 3.4 shows the energy of the first transition state for both the 'Coordinating' and 'Non-Coordinating Pathway' containing the $\left(\mathrm{R}_{\boldsymbol{p}}\right)$-FHPC enantiomer. The energy of the first transition state when the starting material was coordinated to copper was $428.0 \mathrm{kcal} \mathrm{mol}^{\mathbf{- 1}}$. This was a much higher energy barrier than that of the 'Non-Coordinating Pathway'. This large energy difference of $398.6 \mathrm{kcal} \mathrm{mol}^{\mathbf{- 1}}$ indicated that the 'Copper Coordinating Pathway' was not accessible especially as an alternative pathway, the 'Non-Coordinating Pathway', was available which was much lower in energy. 


\subsection{Thermodynamics}

The Gibbs Free Energy, $\Delta \mathrm{G}$, was determined for the overall reaction to see how feasible the reaction for each enantiomer was. Table 4.3 shows the overall Gibbs Free Energy for each reaction. $\Delta \mathrm{G}$ was found to be $2.9 \mathrm{kcal} \mathrm{mol}^{\mathbf{- 1}}$ for the reaction containing $\left(\mathrm{R}_{\boldsymbol{p}}\right)$-FHPC and $3.0 \mathrm{kcal} \mathrm{mol}{ }^{-\mathbf{1}}$ for the reaction containing $\left(\mathrm{S}_{\boldsymbol{p}}\right)$-FHPC. The values are both positive and differ by only $0.1 \mathrm{kcal} \mathrm{mol}^{-1}$. This positive energy indicates the reaction is nonspontaneous. Since the values are only slightly positive the reaction is still able to occur as long as energy is put into the reaction.

\begin{tabular}{lc}
\hline Enantiomer of FHPC & Overall $\boldsymbol{\Delta} \mathrm{G}\left(\mathrm{kcal} \mathrm{mol}^{\mathbf{- 1}}\right)$ \\
\hline $\mathrm{R}_{\boldsymbol{p}}$ & 2.9 \\
$\mathrm{~S}_{\boldsymbol{p}}$ & 3.0 \\
\hline
\end{tabular}

TABLE 3.5: The Gibbs Free Energies, $\Delta \mathrm{G}$, for the overall reaction are reported for both enantiomers.

\subsection{Solvent Effects}

As the copper complexes were unstable in the gaseous phase it was decided to determine their energies in a variety of solvents. The resolution method employed by Rozenberg et al. used the solvent 2-propanol. ${ }^{28}$ Consequently this was one of the solvents investigated. The work carried out by Xin et al. tested ethanol, THF, and $\mathrm{CH}_{2} \mathrm{Cl}_{2}$ as solvents for the asymmetric Henry reaction. It was determined that ethanol was the most suitable solvent for the formation of copper complexes for this type of reaction. ${ }^{36}$ Since the complexes in the resolution method investigated here contain copper these three solvents were investigated along with 2-propanol. Single point calculations were carried out to see what the energy of both the $\left(\mathrm{R}_{\boldsymbol{p}}\right)$ FHPC containing complex and the $\left(\mathrm{S}_{\boldsymbol{p}}\right)$-FHPC containing complex would be in different solvents.

For all three of the solvents suggested by Xin et al. the complexes were stabilised. ${ }^{36}$ EtOH was found to be the most stabilising for both complexes. 2-propanol was $2.0 \mathrm{kcal} \mathrm{mol}{ }^{-1}$ higher in energy for both complexes followed by $\mathrm{CH}_{2} \mathrm{Cl}_{2}$ and THF. THF was the least stabilising out of all the solvents and was $16.0 \mathrm{kcal} \mathrm{mol}^{-1}$ higher in energy. The gas phase structure was the highest at around $66.4 \mathrm{kcal} \mathrm{mol}^{-1}$. 


\begin{tabular}{lcc}
\hline Diastereomer & Solvent & Relative Energy $\left(\mathrm{kcal} \mathrm{mol}^{\mathbf{- 1}}\right)$ \\
\hline$\left(\mathrm{R}_{\boldsymbol{p}}, \mathrm{S}, \mathrm{S}\right)$ & $\mathrm{None}$ & 66.5 \\
& $\mathrm{CH}_{\mathbf{2}} \mathrm{Cl}_{\mathbf{2}}$ & 11.0 \\
& $\mathrm{EtOH}$ & 0.0 \\
& $\mathrm{THF}$ & 16.0 \\
& 2 -propanol & 2.0 \\
\hline$\left(\mathrm{S}_{\boldsymbol{p}}, \mathrm{S}, \mathrm{S}\right)$ & $\mathrm{None}$ & 66.4 \\
& $\mathrm{CH}_{\mathbf{2}} \mathrm{Cl}$ & 11.1 \\
& EtOH & 0.0 \\
& $\mathrm{THF}$ & 16.0 \\
& $2-$ propanol & 2.0 \\
\hline
\end{tabular}

TABLE 3.6: Electronic energies of Min5, the diastereomeric copper complexes, in a variety of solvents were determined.

\subsection{Discussion}

For this resolution method two possible mechanisms have been investigated. Accordingly this section will explore both mechanisms in turn. Subsequently a comparison will be made to endeavour to provide an answer as to which mechanism is more likely to occur.

\subsubsection{N-deprotonation Pathway}

The first energy barrier for TS1 was $30.8 \mathrm{kcal} \mathrm{mol}^{-\mathbf{1}}$ for the $\left(\mathrm{S}_{\boldsymbol{p}}\right)$-FHPC containing diastereomer, and $29.4 \mathrm{kcal} \mathrm{mol}^{-1}$ for the $\left(\mathrm{R}_{\boldsymbol{p}}\right)$-FHPC containing diastereomer. This was a minor energy difference of $1.4 \mathrm{kcal} \mathrm{mol}^{-1}$. In Min1 the $\left(\mathrm{R}_{p}\right)$-FHPC containing diastereomer was again lower in energy, $-4.0 \mathrm{kcal} \mathrm{mol}^{-1}$, compared to the $\left(\mathrm{S}_{\boldsymbol{p}}\right)$-FHPC containing diastereomer, $0.2 \mathrm{kcal} \mathrm{mol}^{-1}$. This was a larger difference of $4.2 \mathrm{kcal} \mathrm{mol}^{-1}$ meaning the two diastereomers differ to a larger degree and thus separation at this step would be more likely.

The small energy difference between the two diastereomers for TS1 can be explained due to the similarities of the transition state. Firstly the amine approached below the cyclophane during the attack. This was because of the second ring in the cyclophane which shielded the other side from being attacked. Secondly the amine was very flexible. Thus, it was able to rotate and thereby reduce the energy if an unfavourable interaction were to occur. In Figure 3.2 it can be seen that the 
two isopropyl groups in (S)-valyl-(S)-valine had rotated to accommodate the cyclophane backbone, the carbonyl of the cyclophane, and the nitrogen of the amine. The configuration of the chiral centre formed here matched the configuration of the cyclophane. Thus a $(\mathrm{S})$ chiral centre was formed for the diastereomer containing $\left(\mathrm{S}_{\boldsymbol{p}}\right)$-FHPC and a $(\mathrm{R})$ chiral centre was formed for the $\left(\mathrm{R}_{\boldsymbol{p}}\right)$-FHPC containing diastereomer.

For the first minimum an energy difference about three times larger than that of the first transition state was seen. This was most likely due to the $\left(\mathrm{R}_{p}\right)$-FHPC containing diastereomer the (S)-valyl-(S)-valine portion of the molecule arranging itself to be nearly planar. Most of the bonds in the molecule were in line with the lower ring of the cyclophane. Thus the distance between the cyclophane backbone and the chain was at its maximum. For the $\left(\mathrm{S}_{\boldsymbol{p}}\right)$-FHPC containing diastereomer part of the (S)-valyl-(S)-valine portion was forced under the cyclophane and the configuration of the resulting chiral centre was S. The two parts of the molecule were thereby closer to each other and this caused the energy of this minimum to be higher.

As mentioned previously TS1 was found to be concerted. Here, the hydrogen migrated from the nitrogen of $(\mathrm{S})$-valyl-(S)-valine to the oxygen of the aldehyde in the cyclophane. This occured at the same time as a bond formed between the nitrogen and carbon. This proceeded along with the breaking of the carbonoxygen double bond to give a single bond. The concerted process prevented the formation of formally charged species. This would therefore be the most likely transition state because the calculations were carried out in the gas phase. Thus there was no solvent present to stabilise the charge. If a polar solvent were incorporated TS1 would, potentially, no longer be concerted. It would be likely that the carbon nitrogen bond would form without the hydrogen migration. The nitrogen would thus be formally positively charged, and the oxygen of the carbonyl formally negatively charged. A polar solvent could stabilise these charges. A polar protic solvent could further stabilise the molecule due to the potential of hydrogen bonding to different areas of the molecule.

For the resolution method employed by Rozenberg et al. 2-propanol was used as the solvent. ${ }^{28}$ This was a polar protic solvent. The first transition state for this reaction when the solvent was involved would most likely therefore not be the concerted process found here where the carbon oxygen bond is broken as the 
nitrogen attacks simultaneously as the hydrogen migrates but the single step that was proposed above where the hydrogen migration is a separate step.

TS2 was again a concerted process, which was thought to occur to prevented the formation of formal charges. Incorporating a solvent could change the transition state to a step-wise reaction where the nitrogen carbon double bond would form and $\mathrm{OH}^{-}$would be ejected without the deprotonation step. The solvent would then most likely deprotonate the positive nitrogen to give the product. Similarly for the concerted transition state the hydroxyl group was able to deprotonate the nitrogen because of their proximity.

Min2, which still contained the water molecule, was lower in energy for the $\left(\mathrm{S}_{\boldsymbol{p}}\right)$ FHPC containing diastereomer than for the $\left(\mathrm{R}_{p}\right)$-FHPC containing diastereomer; 0.8 and $7.3 \mathrm{kcal} \mathrm{mol}^{-\mathbf{1}}$ respectively. This difference of $8.1 \mathrm{kcal} \mathrm{mol}^{-\mathbf{1}}$ was relatively large and should allow for easy separation. The removal of the water molecule stabilised the diastereomer containg the $\left(\mathrm{R}_{\boldsymbol{p}}\right)$-FHPC enantiomer, $1.7 \mathrm{kcal} \mathrm{mol}^{-\mathbf{1}}$, and destabilised the $\left(\mathrm{S}_{\boldsymbol{p}}\right)$-FHPC containing diastereomer, $2.3 \mathrm{kcal} \mathrm{mol}^{\mathbf{- 1}}$. This minor energy difference, $0.6 \mathrm{kcal} \mathrm{mol}^{-\mathbf{1}}$, was the smallest energy difference for this mechanism.

Since Min2 contained a water molecule it allowed for hydrogen bonding between the water and the cyclophane diastereomers. For the molecule containing $\left(\mathrm{R}_{\boldsymbol{p}}\right)$ FHPC (Figure 3.10a) the water molecule hydrogen bonded to the hydrogen on the nitrogen in the amide. The cyclophane hydroxyl group formed a hydrogen bond to one of the hydrogens of the water molecule. For the diastereomer containing $\left(\mathrm{S}_{\boldsymbol{p}}\right)$-FHPC the water molecule formed a hydrogen bond to the hydrogen in the cyclophane hydroxyl group and it was the oxygen in the amide that interacted with the hydrogen of the water molecule. The presence of these hydrogen bonds stabilised both molecules. The $\left(\mathrm{S}_{\boldsymbol{p}}\right)$-FHPC containing diastereomer was, however, more stabilised than the $\left(\mathrm{R}_{\boldsymbol{p}}\right)$-FHPC containing diastereomer. This was because the formation of the hydrogen bond forced the molecule into a slightly less desirable conformation even though the formation of the hydrogen bond stabilised the molecule. It does mean, however, that the energy of the two enantiomers was higher.

This is a much larger energy difference, $8.1 \mathrm{kcal} \mathrm{mol}^{-\mathbf{1}}$, compared to that at Min1. The ordering of the two diastereomers is, however, different. For Min1 the $\left(\mathrm{R}_{\boldsymbol{p}}\right)$ FHPC containing diastereomer was lower in energy while for Min2 it was the 
$\left(\mathrm{S}_{\boldsymbol{p}}\right)$-FHPC containing diastereomer. Separation of the two diastereomers would be more likely to occur at Min2 because of this larger energy difference. This energy difference does, however, depend on the presence of the water molecule that was released during TS2. Removal of this water molecule reverses the order of stability to that at Min1. This was because the (S)-valyl-(S)-valine portion was able to rotate and adopt a more stable conformation. Hydrogen bonding, other than between the hydroxyl and the nitrogen of the imine, was no longer possible. The lower energy of the $\left(\mathrm{R}_{\boldsymbol{p}}\right)$-FHPC containing diastereomer suggested that the presence of the water restricted the molecule to adopt a conformation that was unfavourable were it not for the hydrogen bonding. When the water was removed the molecule was able to relax to a more favourable conformation.

Coordination of this product to the copper (II) ion increased the energy dramatically for both enantiomers. The energy of the $\left(\mathrm{R}_{\boldsymbol{p}}\right)$-FHPC containing diastereomer was $375.5 \mathrm{kcal} \mathrm{mol}^{-1}$ while for the $\left(\mathrm{S}_{\boldsymbol{p}}\right)$-FHPC containg diastereomer it was 375.4 kcal $\mathrm{mol}^{-1}$. A reversal, compared to the previous minimum, was seen but the energy difference, $0.1 \mathrm{kcal} \mathrm{mol}^{-1}$, had decreased even further. The separation of these diastereoemric copper complexes would thus be extremely difficult.

The energy of the diastereomeric copper complexes was so similar that separation would be near to impossible. The energy difference can already be increased through the exclusion of copper perchlorate in the reaction and separating the diastereomers at Min3. Here, the energy difference was only $0.7 \mathrm{kcal} \mathrm{mol}^{-\mathbf{1}}$ but this energy difference was already six times larger. Ideally separation would be carried out at Min1 or Min2 since the energy difference was much larger.

The energy between the diastereomers in this reaction could be further increased if the flexibility of the amine were to be restricted. This restriction would prevent the molecule from rotating and thus adopting a lower energy conformation for one diastereomer, and a higher energy conformation for the other. This can be done by introducing a larger group than isopropy groups such as isobutyl or even phenyl groups. An alternative approach would be to use a much smaller amine which would have a smaller number of conformations. This would then ideally increase the energy difference between the diastereomers. 


\subsubsection{O-deprotonation Pathway}

The first transition state and minimum, TS1 and Min1, are indentical for this mechanism, as was discussed for the 'N-Deprotonation Pathway' above. The next transition state, TS2 where the water molecule was removed was very close in energy for the two enantiomer, $21.1 \mathrm{kcal} \mathrm{mol}^{-1}$ for the $\left(\mathrm{R}_{\boldsymbol{p}}\right)$-FHPC containing diastereomer and $21.3 \mathrm{kcal} \mathrm{mol}^{\mathbf{- 1}}$ for the $\left(\mathrm{S}_{\boldsymbol{p}}\right)$-FHPC containing diastereomer. This was a very small energy difference; $0.2 \mathrm{kcal} \mathrm{mol}^{-\mathbf{1}}$. Thus the transition states were almost identical in energy. This was probably due to the newly formed hydroxyl group already being oriented towards the hydrogen of the cyclophane hydroxyl group in Min1 to give a hydrogen bond. Instead of a rotation occuring to allow for the water molecule to leave via the bottom of the cyclophane, the water molecule was ejected from this more shielded side. The (S)-valyl-(S)-valine portion was rotated away to try and reduce sterics and thus make it more feasible for the water molecule to leave. The hydrogen in the isopropyl group was still oriented towards the cyclophane to try and reduce sterics and, at the same time, make it easier for the water molecule to leave. The carbonyl of the amide was now oriented completely away from the two oxygens and even the nitrogen. Thus the sterics were reduced and there was no interaction between the oxygens. The isopropyl group on the amide portion was now oriented away from the cyclophane so that the hydrogen on the carbon next to the nitrogen in the amide was oriented further towards the cyclophane. Once again this opened up space for the forming water molecule to leave and thus prevented an increase in energy.

The resulting minimum, Min2, had, however, a very big energy difference of 15.9 kcal mol ${ }^{-1}$. The $\left(\mathrm{R}_{p}\right)$-FHPC containing diastereomer was greatly stabilised in this minimum with an energy of $-8.5 \mathrm{kcal} \mathrm{mol}^{-\mathbf{1}}$ particularly when compared to that of the $\left(\mathrm{S}_{\boldsymbol{p}}\right)$-FHPC containing diastereomer, $7.4 \mathrm{kcal} \mathrm{mol}^{-1}$. The diastereomers would therefore be relatively easily separated as long as the reaction does not continue. When the water molecule was removed the energy of the $\left(\mathrm{R}_{p}\right)$-FHPC containing diastereomerwas greatly increased to $10.5 \mathrm{kcal} \mathrm{mol}^{-\mathbf{1}}$ while the energy of the $\left(\mathrm{S}_{\boldsymbol{p}}\right)$ FHPC containing diastereomer dropped to $3.2 \mathrm{kcal} \mathrm{mol}^{-1}$ resulting in a difference of $7.3 \mathrm{kcal} \mathrm{mol}^{-1}$.

The large energy difference for Min2 was most likely a result of there being more hydrogen bonds in the $\left(\mathrm{R}_{\boldsymbol{p}}, \mathrm{R}, \mathrm{S}, \mathrm{S}\right)$ - than in the $\left(\mathrm{S}_{\boldsymbol{p}}, \mathrm{S}, \mathrm{S}, \mathrm{S}\right)$-diastereomer. In the 
$\left(\mathrm{R}_{p}, \mathrm{R}, \mathrm{S}, \mathrm{S}\right)$-diastereomer there was a hydrogen bond between the formally negative oxygen and hydrogen of the water molecule and another to the hydrogen on the nitrogen of the imine. The oxygen in the water molecule also formed a hydrogen bond to the hydrogen of the nitrogen in the amide. For the $\left(\mathrm{S}_{p}, \mathrm{~S}, \mathrm{~S}, \mathrm{~S}\right)$ diastereomer the only hydrogen bond present was between the formally negatively charged oxygen and a hydrogen of the water molecule.

For Min3 the energy had increased by $19.0 \mathrm{kcal} \mathrm{mol}^{\mathbf{- 1}}$ when the water molecule was removed. This large energy change was due to the large number of hydrogen bonds that were no longer present due to the removal of the water molecule. Hydrogen bonding was still present in Min3; between the formally negative oxygen and the hydrogen of the nitrogen of the imine as well as to the hydrogen on the nitrogen of the amide. The formation of the hydrogen bond to the nitrogen of the amide resulted in a conformation that was higher in energy because the (S)-valyl-(S)-valine part of the molecule was now closer to the cyclophane and thereby destabilised the molecule. For the $\left(\mathrm{S}_{p}, \mathrm{~S}, \mathrm{~S}, \mathrm{~S}\right)$-diastereomer the energy had decreased by $4.2 \mathrm{kcal} \mathrm{mol}^{-\mathbf{1}}$. This was most likely due to the fact that the imine had rotated so it was now in the cis-like conformation which allowed for the formation of a hydrogen bond between the hydrogen on the nitrogen of the imine and the formally negatively charged oxygen. For the $\left(\mathrm{R}_{p}, \mathrm{R}, \mathrm{S}, \mathrm{S}\right)$-diastereomer this rotation occured during the second transition state which was why it was stabilised. The extra stabilisation when the water molecule was removed is the reason why the energy was lower for the $\left(\mathrm{S}_{p}, \mathrm{~S}, \mathrm{~S}, \mathrm{~S}\right)$-diastereomer.

The final transition state, TS3, had a very small energy barrier for both reactions; the energy difference for the two diastereomers was, however, much larger at 6.3 $\mathrm{kcal} \mathrm{mol}{ }^{-1}$. This energy difference was close to the largest difference observed for the previous mechanism. The barrier for the $\left(\mathrm{S}_{p}\right)$-FHPC containing diastereomer was higher, at $2.5 \mathrm{kcal} \mathrm{mol}^{-1}$, compared to the barrier for the $\left(\mathrm{R}_{p}\right)$-FHPC containing mechanism which was nearly half the size, at $1.4 \mathrm{kcal} \mathrm{mol}^{-1}$.

The energy of the $\left(\mathrm{R}_{p}\right)$-FHPC containing diastereomer dropped below that of the $\left(\mathrm{S}_{p}\right)$-FHPC containing diastereomer for Min4; $1.7 \mathrm{kcal} \mathrm{mol}^{-1}$ for the reaction containing $\left(\mathrm{R}_{p}\right)$-FHPC and $2.3 \mathrm{kcal} \mathrm{mol}^{-1}$ for the $\left(\mathrm{S}_{\boldsymbol{p}}\right)$-FHPC enantiomer. The energy of the copper complexes was identical as for the previous pathway.

During the third transition state, TS3, the hydrogen migrated from the nitrogen to the oxygen. As the hydrogen was already in the ideal location during Min3 this 
explains why there was such a small barrier to reaching Min4. The transition state containg $\left(\mathrm{S}_{\boldsymbol{p}}\right)$-FHPC was lower in energy due to the (S)-valyl-(S)-valine portion of the molecule being below the cyclophane. For the $\left(\mathrm{R}_{p}\right)$-FHPC containing molecule this portion was still rotated upwards to allow for the formation of a hydrogen bond between the hydrogen on the nitrogen of the amide and the formally negatively charged oxygen.

This hydrogen bond would most likely still be present in order to stabilise the molecule during TS3. If the (S)-valyl-(S)-valine portion where to rotate below the cyclophane the molecule could be further destabilised and thus raise the energy further.

The $\left(\mathrm{R}_{p}, \mathrm{R}, \mathrm{S}, \mathrm{S}\right)$-diastereomer was now lower in energy than the $\left(\mathrm{S}_{p}, \mathrm{~S}, \mathrm{~S}, \mathrm{~S}\right)$ diastereomer in Min4. The energy difference was very small and thus separation here would be very difficult. The $\left(\mathrm{R}_{p}\right)$-FHPC containing reaction was now lower in energy because the (S)-valyl-(S)-valine portion was no longer forced to adopt a position where it was directly next the cyclophane. This portion of the molecule relaxed to be more elongated and thus increase the distance. For the reaction containing the $\left(\mathrm{S}_{\boldsymbol{p}}\right)$-FHPC enantiomer the molecule had not rotated. The molecule was stabilised compared to the previous minimum due to no formal charges being present and the oxygen, which is more electronegative, now being protonated again.

\subsubsection{Comparison}

To determine which mechanism is more likely to occur depends on the barriers that need to be overcome and how stable the products are. Since the first transition state and minimum, TS1 and Min1 respectively, are identical for both mechanisms, along with the product, it is only the steps in between that are important in determining the most likely pathway.

The two pathways deviated at the second transition state, TS2, and therefore this was the most important step for trying to determine which pathway was more likely to occur. The energy barrier for the 'N-deprotonation Pathway' was more than twice the size of the 'O-deprotonation Pathway'; approximately 55.4 and $21.2 \mathrm{kcal} \mathrm{mol}^{-1}$. Due to this large barrier the reaction would proceed through the 'O-deprotonation Pathway'. 
The second minimum for the 'O-Deprotonation Pathway' was, however, relatively high in energy for the $\left(\mathrm{S}_{\boldsymbol{p}}\right)$-FHPC containing diastereomer; $7.4 \mathrm{kcal} \mathrm{mol}^{\mathbf{- 1}}$. Even though the transition state was much higher for the 'N-Deprotonation Pathway' the resulting minimum was much lower in energy; $-0.8 \mathrm{kcal} \mathrm{mol}^{\mathbf{- 1}}$. This large difference in stability of the two minima opens up the possibility of kinetic and thermodynamic control in the reaction. If the temperature were kept low throughout the reaction the $\left(\mathrm{S}_{\boldsymbol{p}}\right)$-FHPC containing diastereomer would proceed through the 'O-Deprotonation Pathway' because of the lower energy barrier. If, however, the temperature were kept high and the reaction time kept long the 'N-Deprotonation Pathway' becomes accessible and the more thermodynamic product will be formed.

The opposite is true for the $\left(\mathrm{R}_{\boldsymbol{p}}\right)$-FHPC containing diastereomer. Here, Min2 was lower in enegy for the 'O-deprotonation Pathway'; $-8.5 \mathrm{kcal} \mathrm{mol}^{-\mathbf{1}}$. Min2 for the 'N-deprotonation Pathway' was was much higher in energy at $7.3 \mathrm{kcal} \mathrm{mol}^{-\mathbf{1}}$. The 'O-deprotonation Pathway' is therefore preferred kinetically, due to the low energy barrier of TS1, and thermodynamically, due to the greater stability of Min2.

The product for both these pathways are identical and therefore kinetic and thermodynamic control is only important if the reaction is not allowed to go to completion. This is because the two enantiomers of FHPC are unable to form another product other than the final imine. The energy difference of the end product, both the copper complexes and the imine, are too similar in energy to be able to separate the diastereomers with ease. If a different end point were chosen in the reaction separation could become easier. The energy difference between the two diastereomers the largest for the 'O-Deprotonation Pathway' for Min2. This indicates that if the reaction can be stopped here separation should be easier.

During the resolution method proposed by Rozenberg et al. the reaction mixture was refluxed for 3 hours before it was cooled down and left overnight at room temperature. ${ }^{28}$ Since the reaction was refluxed for a relatively long time, high energy barriers become accessible along with the more thermodynamic product. The method used therefore establishes access to both pathways, but it would still be more feasible that the 'N-Deprotonation Pathway' would occur. The 'NDeprotonation Pathway' typically contained smaller energy differences between the two diastereomers. The separation of these diastereomers would therefore be much harder than if the reaction had gone via the 'O-Deprotonation Pathway'. Experimentally the separation could be improved if the temperature were kept lower so that the 'O-Deprotonation Pathway' becomes the more probable pathway. 
This way greater energy differences would occur throughout the reaction and if the reaction were to be stopped before the formation of the imine the separation could be greatly improved.

In light of the fact that the two copper complexes are so similiar in energy this explains why an alternative resolution method was proposed a year later by Rozenberg and co-workers. ${ }^{39}$ This alternative method will be discussed in the next chapter.

The rate determining step for the 'N-Deprotonation Pathway' was TS2 while for the 'O-Deprotonation Pathway' it was TS1 because it had the largest energy barrier.

\subsubsection{Copper Coordinating Pathway}

For the 'Copper Coordinating Pathway' the energy of the first transition state was much higher than that of the 'Non-Coordinating Pathway'. The coordination to the copper ion forced the carboxylic acid group closer to the cyclophane compared to the 'Non-Coordination Pathway'. This raised the energy of the entire structure. Combined with the fact that the coordination to the copper forced the molecule into a conformation that was not flexible this raised the energy further.

For the 'Non-Coordinating Pathway' the long carbon chain of (S)-valyl-(S)-valine provided flexibility which could help stabilise the molecule through hydrogen bonding and decreasing the distance between unfavourable groups. The presence of the copper ion forced the molecule into a fixed geometry that cannot easily accomodate changes. The energy of this complex was therefore much higher in energy. Since this first transition state was so much higher in energy for the 'Copper Coordinating Pathway' it can be concluded that this was not the mechanism that occured. Instead the reaction would proceed through the 'Non-Coordinating Pathway'.

\subsubsection{Thermodynamics}

$\Delta \mathrm{G}$ for the overall reaction was found to be positive for both enantiomers of FHPC at room temperature. The reaction is therefore nonspontaneous when carried out in the gas phase meaning energy would be required for this reaction to occur when 
carried out at room temperature. The actual reaction carried out by Rozenberg et $a l$. was refluxed for three hours and was spontaneous. ${ }^{28}$ The extra energy provided by heating the solution therefore allowed the reaction to proceed.

The energy difference between the diastereomers remained small even when thermodynamics was taken into consideration. This once again shows how difficult separation would be at the end of the reaction.

\subsubsection{Solvent Effects}

It was found that both copper complex were the most stable in ethanol. Even though the complex was slighly less stable in 2-propanol compared to EtOH this does not automatically make it was a better solvent. This only indicates that for this structure EtOH was the solvent that stabilised the complex the most. The structures were not optimised and therefore more suitable conformations were not able to be adopted. If the structures were allowed to relax a greater energy difference could potentially be seen and thus give further insight into the formation of these copper complexes.

The structure in the gas phase had the highest energy because the negative charge of the complex cannot be stabilised here. Ethanol and 2-propanol were able to stabilise the complexes to the greatest extent due to them being polar protic solvents. The polarity of the solvent will stabilise the negatively charged complexes. Since ethanol and 2-propanol are able to hydrogen bond the complexes would be furhter stabilised. Ethanol would stabilise the molecule further than 2-propanol because it is a smaller alcohol and therefore steric interactions would be reduced. THF and $\mathrm{CH}_{2} \mathrm{Cl}_{2}$ are not able to hydrogen bond and therefore would not stabilise the complexes to the same extent.

The energy differences betweent the two diastereomeric copper complexes was not increased when solvent effects were taken into consideration. The energy difference even decreased for all solvent except $\mathrm{CH}_{2} \mathrm{Cl}_{2}$ were it remained at $0.1 \mathrm{kcal} \mathrm{mol}^{-1}$ as with the gas phase. The complexes might therefore be even harder to separate when a solvent is present. 


\section{Chapter 4}

\section{Results and Discussion (Resolution 2)}

The mechanism for the second resolution method is, for the most part, identical to the first. It does not differ in the transition states and intermediates but rather in the orientation adopted during these steps. This is because this resolution method utilised a different amine but the product was still an imine. Antonov et al. proposed a resolution of FHPC using (R)- $\boldsymbol{\alpha}$-PEAM instead of (S)-valyl-(S)valine. ${ }^{39}$ The structure of the two amines is shown in Figure 4.1. (R)- $\boldsymbol{\alpha}$-PEAM is a much smaller amine in terms of atom number, but it is a much bulkier amine due to the inclusion of a phenyl group.<smiles>CC(C)[C@H](N)C(=O)N[C@@H](C(=O)O)C(C)C</smiles><smiles>C[C@H](N)c1ccccc1</smiles>

Figure 4.1: Structure of (S)-valyl-(S)-valine and (R)- $\boldsymbol{\alpha}$-PEAM, the amines used in the first and second resolution method respectively.

For this resolution method the same two deprotonations are possible during the second transition state as with the previous resolution method; deprotonation of the nitrogen of the imine or the cyclophane hydroxyl group. Both of these pathways have been considered for this resolution method and will be presented 
separately. The same naming scheme has been applied here as in the previous chapter.

\subsection{N-deprotonation Pathway Overview}

The first step of the reaction involved the attack of the carbonyl carbon of the cyclophane by the nitrogen from (R)- $\boldsymbol{\alpha}$-PEAM (TS1, Figure 4.2). This occured simultaneously as the carbonyl oxygen deprotonated the nitrogen of (R)- $\boldsymbol{\alpha}$-PEAM. The nitrogen-hydrogen bond was consequently broken. This concerted step prevented the formation of formally charged species; a negatively charged oxygen and a positively charged nitrogen. The step-wise transition state was searched for but was unable to be found.

In the resulting minimum (Min1, Figure 4.2) a hydroxyl group was produced which was accompanied by the formation of a bond between the nitrogen and the carbon of the former carbonyl. The hydrogen on the hydroxyl group was oriented away from the cyclophane hydroxyl group. This allowed for the formation of a hydrogen bond between the oxygen of the newly formed hydroxyl group and hydrogen of the cyclophane hydroxyl group. This interaction stabilised the diastereomers.

This newly formed hydroxyl group was then ejected through the formation of a water molecule (TS2, Figure 4.2). The carbon-oxygen bond was broken due to the formation of a double bond between the nitrogen and the carbon. This ejected hydroxyl group then deprotonated the nitrogen.

In the minimum that followed, a hydrogen bond had been formed between a hydrogen of the water molecule and the nitrogen of the imine (Min2, Figure 4.2). The minimum was stabilised as a result of the formation of this hydrogen bond. The final product was then produced through the removal of the water molecule (Min3, Figure 4.2). Here the imine was rotated in towards the cyclophane hydroxyl group. This allowed for the formation of a hydrogen bond between the nitrogen and the hydrogen of the cyclophane hydroxyl group thus stabilising the product. 


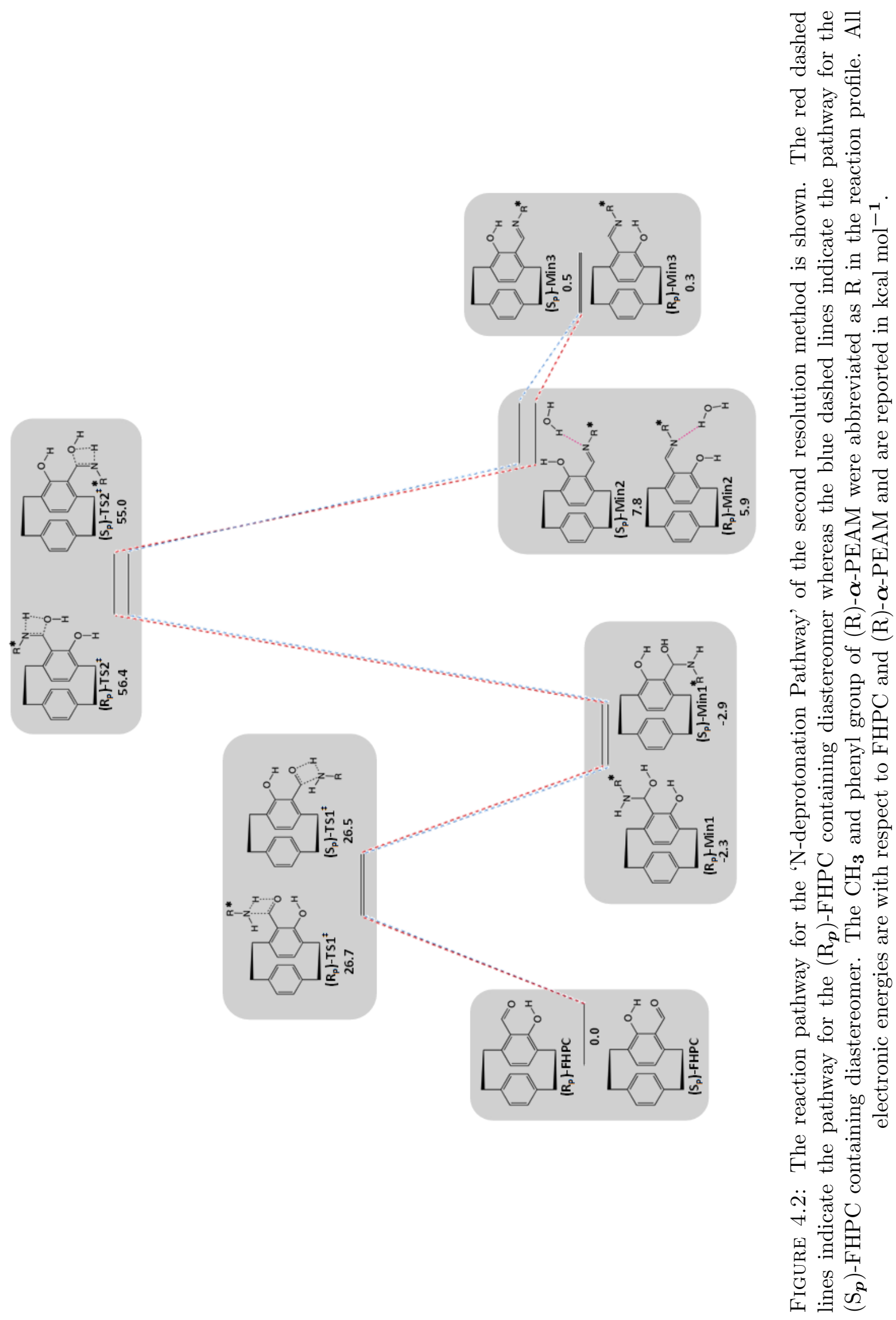




\subsubsection{Transition State 1}

Figure 4.3 shows the 3 -dimensional structures of FHPC, the first transition state (TS1), and the first minimum (Min1) for both enantiomers of FHPC. During the first transition state the primary amine attacked the carbonyl carbon from below the cyclophane. Attack occured below the plane of the cyclophane that does not contain the substituted ring. This is because it is the less hindered side due to the stacked rings of the cyclophane. The hydrogen on the tertiary carbon of (R)$\boldsymbol{\alpha}$-PEAM was oriented slightly towards the cyclophane. This reduced sterics and thus lowered the energy. The $\mathrm{CH}_{\mathbf{3}}$ group on this tertiary carbon was consequently forced closer to the cyclophane to increase the distance betweeen the phenyl group of (R)- $\boldsymbol{\alpha}$-PEAM and the cyclophane. The molecule was thereby stabilised.

No formal charges were formed during TS1 due to the transition state being a concerted process. In the resulting minimum (Min1, Figure 4.3) the (R)- $\boldsymbol{\alpha}$ PEAM portion was oriented away from the cyclophane and the newly formed hydroxyl group. This prevented a detabilising interaction of the bulky phenyl ring and the rings of the cyclophane. The hydrogen on the nitrogen was oriented away from the hydrogen of the newly formed hydroxyl group. This was owing to the fact that a hydrogen bond had formed between the cyclophane hydroxyl hydrogen and the oxygen of the newly formed hydroxyl group. This hydrogen bond would stabilise the molecule.

Figure 4.3 also shows the electronic energies of this step with respect to the cyclophane. The first transition state represents a reasonablly large barrier of around $26.0 \mathrm{kcal} \mathrm{mol}^{-\mathbf{1}}$. The resulting minimum was much lower in energy and is stabilised by $-2.0 \mathrm{kcal} \mathrm{mol}^{-1}$ compared to the cyclophane.

\subsubsection{Rotational Barrier}

The rotational barrier for converting from the cis-like conformer to the translike conformer was carried out as mentioned in the previous chapter. The cis-like conformer was found to be the most stable conformer for both diastereomers. This was because hydrogen bonding between the hydrogen of the cyclophane hydroxyl group and the nitrogen of the imine was possible. The trans-like conformer was found to be higher in energy; 13.8 and $13.9 \mathrm{kcal} \mathrm{mol}^{-\mathbf{1}}$ for the $\left(\mathrm{S}_{\boldsymbol{p}}\right)$-FHPC and $\left(\mathrm{R}_{p}\right)$-FHPC containing diastereomer respectively. 
a)
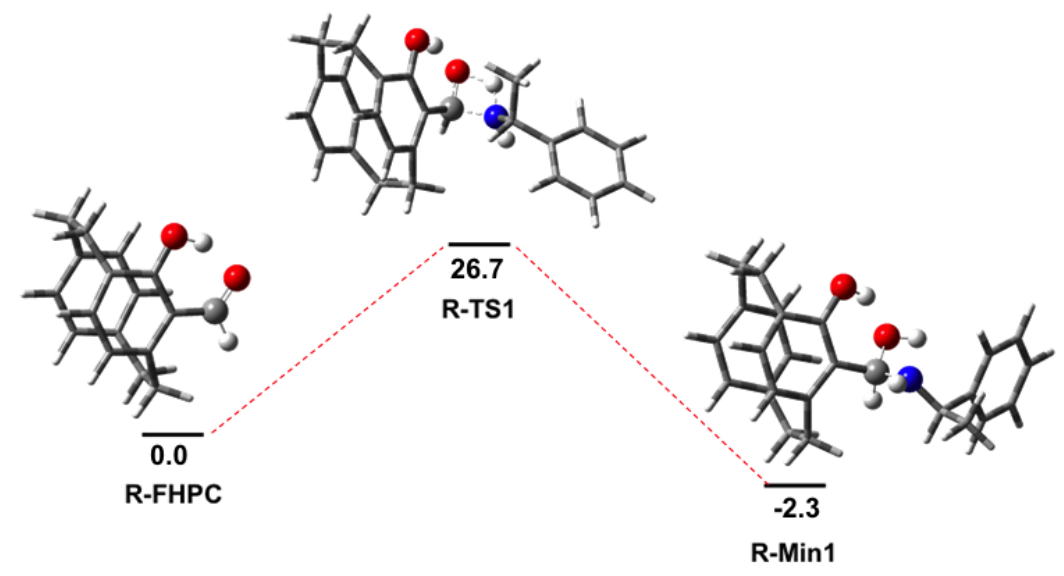

b)
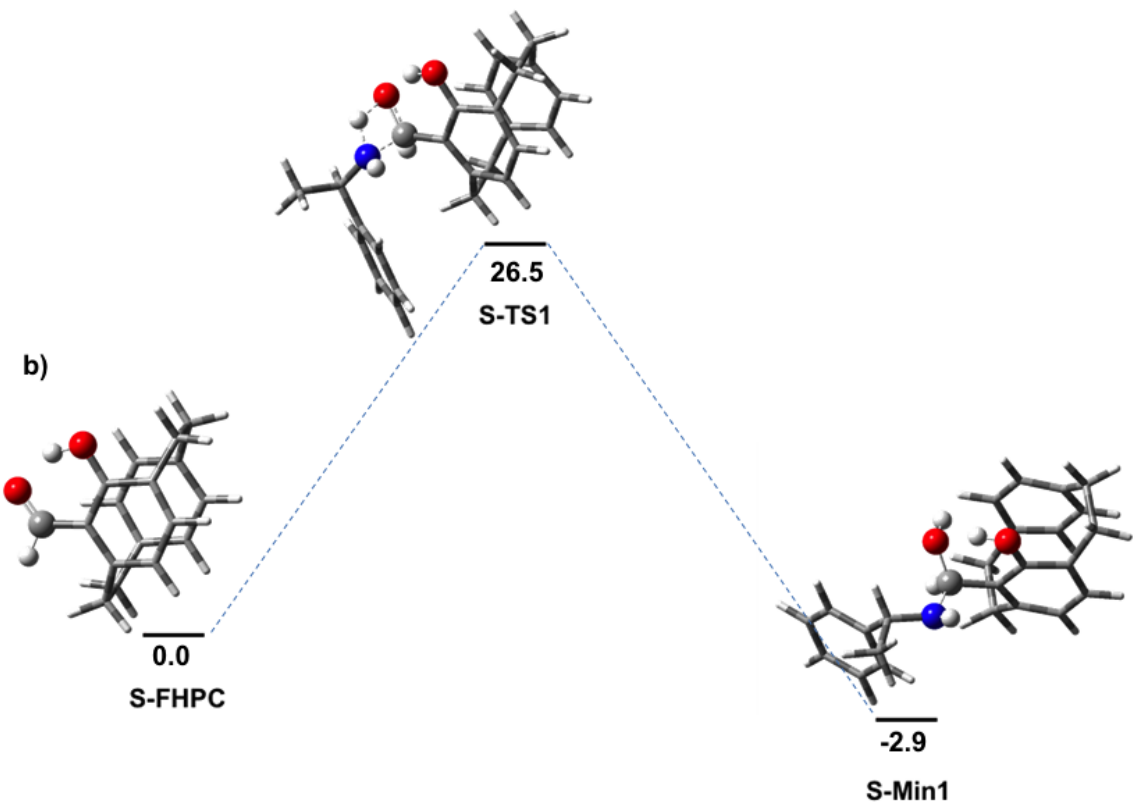

Figure 4.3: The structures of FHPC, TS1, and Min1 for the $\left(\mathrm{R}_{p}\right.$-FHPC containing diastereomer a) and the $\left(\mathrm{S}_{\boldsymbol{p}}\right.$-FHPC containing diastereomer $\left.\mathrm{b}\right)$ are shown. The white, grey, red and blue atoms are $\mathrm{H}, \mathrm{C}, \mathrm{O}$, and $\mathrm{N}$ respectively. The electronic energies are with respect to the starting material, FHPC and (R)- $\boldsymbol{\alpha}$-PEAM, and are reported in $\mathrm{kcal} \mathrm{mol}^{-1}$.

Converting from the less stable trans-like conformer to the more stable cis-like conformer required $2.8 \mathrm{kcal} \mathrm{mol}^{-\mathbf{1}}$ for the $\left(\mathrm{S}_{\boldsymbol{p}}\right)$-FHPC containing diastereomer while for the $\left(\mathrm{R}_{p}\right)$-FHPC containing diastereomer the barrier was lower at $2.1 \mathrm{kcal}$ $\mathrm{mol}^{-\mathbf{1}}$. The trans-like conformer can therefore easily rotate to adopt the more stable conformation. The barrier for converting from the cis-like to the translike conformer was much higher at 16.6 and $16.0 \mathrm{kcal} \mathrm{mol}^{-\mathbf{1}}$ for the $\left(\mathrm{S}_{\boldsymbol{p}}\right)$-FHPC and $\left(\mathrm{R}_{\boldsymbol{p}}\right)$-FHPC containing diastereomer respectively. Molecules in the cis-like 


\begin{tabular}{llc}
\hline Intermediate & Orientation & Relative Energy $\left(\mathrm{kcal} \mathrm{mol}^{\mathbf{- 1}}\right)$ \\
\hline$\left(\mathrm{S}_{\boldsymbol{p}}\right)$-Min3 & cis-like & 0.0 \\
& trans-like & 13.8 \\
& TS & 16.6 \\
& Barrier $_{\text {trans } \rightarrow \text { cis }}$ & 2.8 \\
& Barrier $_{\text {cis } \rightarrow \text { trans }}$ & 16.6 \\
\hline$\left(\mathrm{R}_{\boldsymbol{p}}\right)$-Min3 & cis-like & 0.0 \\
& trans-like & 13.9 \\
& TS & 16.0 \\
& Barrier $_{\text {trans } \rightarrow \text { cis }}$ & 2.1 \\
& Barrier $_{\text {cis } \rightarrow \text { trans }}$ & 16.0 \\
\hline
\end{tabular}

TABLE 4.1: The rotational barrier for converting from the cis-like conformation, imine rotated towards the hydroxyl group, to the trans-like conformation, imine rotated away from the hydroxyl group, was determined for both diastereomers in the reaction; $\left(\mathrm{S}_{\boldsymbol{p}}\right)$-Min3 contains the $\left(\mathrm{S}_{\boldsymbol{p}}\right)$-FHPC enantiomer and $\left(\mathrm{R}_{\boldsymbol{p}}\right)$-Min3 contains the $\left(\mathrm{R}_{p}\right)$-FHPC enantiomer. The dihedral angle with respect to the plane created by the two carbons of the cyclophane ring and the plane created by the carbon and nitrogen of the imine was varied.

conformation would therefore not be likely to convert to the less stable trans-like conformer because of this larger barrier.

\subsubsection{Transition State 2}

Figure 4.4 shows the structure of Min1 along with the final transition state and the minimum leading to the product. In the second transition state, TS2, the water molecule was ejected via the same path as the nitrogen previously attacked i.e. below the cyclophane. This occured by deprotonation of the nitrogen and resulted in the formation of an imine. A more planar geometry had already been adopted with respect to the carbon, nitrogen, and hydrogen of the resulting imine. For TS2 to occur an energy barrier of about $57.0 \mathrm{kcal} \mathrm{mol}^{\mathbf{- 1}}$ had to be overcome. This was much larger than the barrier of TS1 which was only $26.0 \mathrm{kcal} \mathrm{mol}^{\mathbf{1}}$.

The subsequent minimum (Min2, Figure 4.4) was stabilised by a hydrogen bond between the nitrogen of the imine and hydrogen of the water molecule due to the imine having adopted a cis-like conformation. The water molecule in this minimum was no longer below the cyclophane but adjacent to it. This minimum was around 49.0 kcal mol ${ }^{-1}$ lower in energy than the transition state. The $\mathrm{CH}_{\mathbf{3}}$ group of (R)$\boldsymbol{\alpha}$-PEAM had undergone a rotation and was now directed towards the cyclophane. 
This opened up space for the water molecule to occupy the position adjacent to the cyclophane without greatly destabilising the molecule.

The removal of the water molecule lead to the product (Min3, Figure 4.4) which was further stabilised by around $6.0 \mathrm{kcal} \mathrm{mol}^{-\mathbf{1}}$. The $\mathrm{CH}_{\mathbf{3}}$ group had now rotated away from the cyclophane due to sterics. The cis-like orientation of the imine allowed for a hydrogen bond to form between the hydrogen on the hydroxyl group and the nitrogen of the imine.

\section{$4.2 \quad$ O-deprotonation Pathway Overview}

The 'O-deprotonation Pathway' for this resolution method proceeded through the identical first transition state, TS1, first minimum, Min1, and ended with the identical final product as the 'N-deprotonation Pathway'. The pathway deviated as a result of the possibility of two deprotonation sites during the second transition state. The hydroxyl group that was ejected during TS2 can deprotonate the nitrogen of the imine to give the desired product ('N-deprotonation Pathway') or the cyclophane hydroxyl group can be deprotonated ('O-deprotonation Pathway'). This deprotonation of the hydroxyl group on the cyclophane was found to occur simultaneously as a double bond formed between the nitrogen and the carbon.

The minimum that followed (Min2, Figure 4.5) consequently contained an imine. The water molecule that was ejected subsequently hydrogen bonded to the formally negatively charged oxygen of the cyclophane thus stabilising the molecule. The water molecule was then removed (Min3, Figure 4.5) and thereby caused the imine to rotate towards the negatively charged oxygen. This rotation enabled the formation of a hydrogen bond between the negatively charged oxygen and the hydrogen on the imine nitrogen.

The proximity of the hydrogen on the nitrogen to the negatively charged oxygen allowed for a hydrogen migration to occur in the final transition state (TS3, Figure 4.5). This returned the cyclophane hydroxyl group and resulted in the formation of the diastereomeric imine product. 


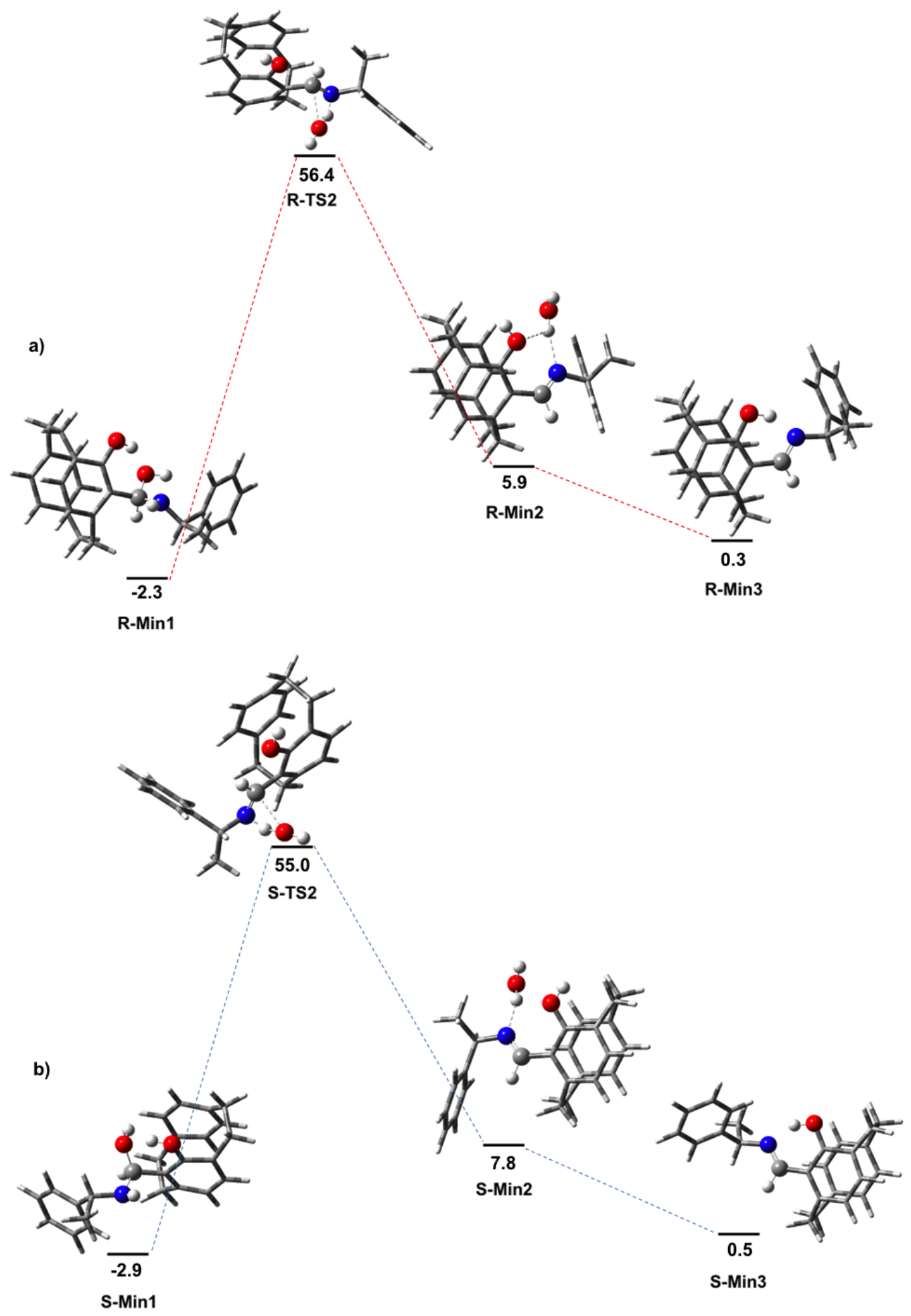

Figure 4.4: The structures of Min1, TS2, and Min2 for the $\left(\mathrm{R}_{p}\right.$-FHPC containing diastereomer a) and the $\left(\mathrm{S}_{\boldsymbol{p}}\right.$-FHPC containing diastereomer b) are shown. The white, grey, red and blue atoms are $\mathrm{H}, \mathrm{C}, \mathrm{O}$, and $\mathrm{N}$ respectively. The electronic energies are with respect to the starting material, FHPC and (R)- $\boldsymbol{\alpha}$-PEAM, and are reported in $\mathrm{kcal} \mathrm{mol}^{-1}$. 
?

品

远

近臣

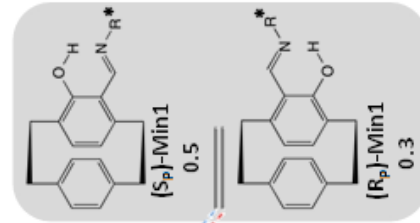

$\dot{1} \Xi$

焉尊

. $\cong$.

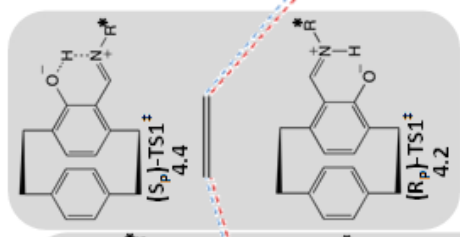

过包

: $:$ 品

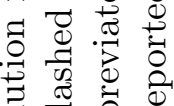

웡

車

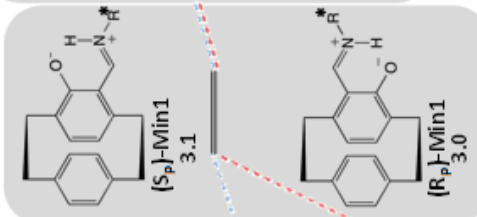

궁효

为主

O

\begin{tabular}{lll}
\hline \\
0
\end{tabular}

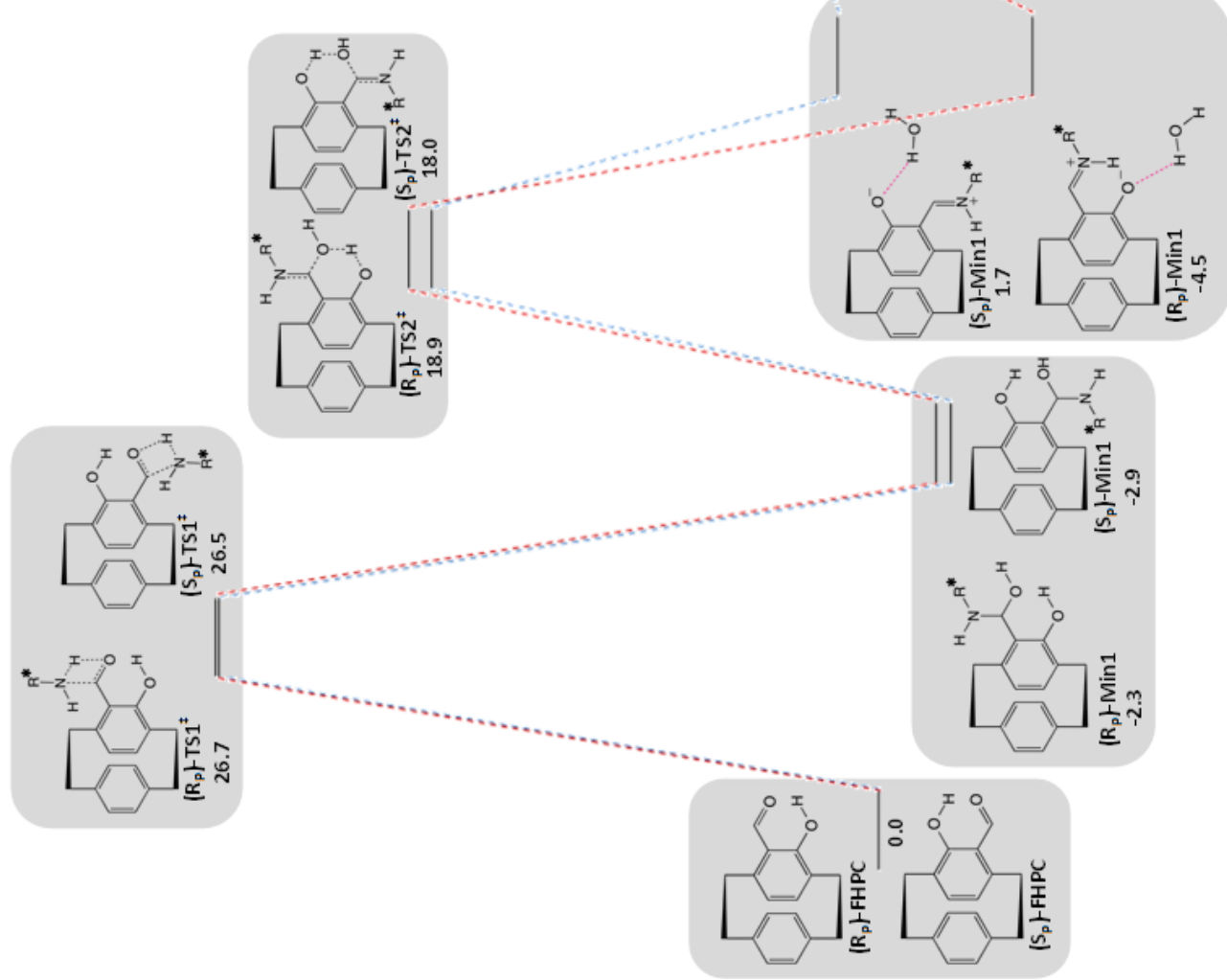

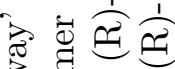

吾荡

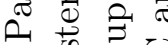

요

焉

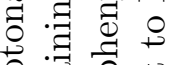

要

원

○四索

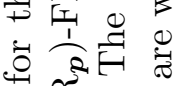

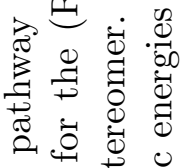

궁. ․ㅗㅇ

范宽

范 $\cdot$ :

包

$\therefore$ ○ 0

में

되

点. 


\subsubsection{Transition State 2}

Min1 proceeded through the second transition state (TS2, Figure 4.6) where the newly formed hydroxyl group was ejected to form the desired imine. As the hydroxyl group was expelled the cyclophane hydroxyl group was deprotonated. The (R)- $\boldsymbol{\alpha}$-PEAM portion of the molecule rotated to accomodate for the hydroxyl group. This was because of the relatively large electron cloud on the oxygen. The phenyl group in (R)- $\boldsymbol{\alpha}$-PEAM therefore rotated away from the hydroxyl group and the unsubstituted ring of the cyclophane, thus orienting itself downward instead. The hydrogen on the carbon of the imine was therefore the largest group oriented towards the water molecule. This reduced bulk around the water stabilised the molecule. The barrier for this transition state was around $20.0 \mathrm{kcal} \mathrm{mol}^{\mathbf{- 1}}$.

This transition state then lead to a minimum about $13.0 \mathrm{kcal} \mathrm{mol}^{\mathbf{- 1}}$ lower in energy (Min2, Figure 4.6). Here, a hydrogen bond was formed between the hydrogen of the water molecule and the cyclophane hydroxyl group. The water molecule was then removed in the following minimum (Min3, Figure 4.6). This resulted in the formation of charges; specifically a formally negatively charged oxygen and a formally positively charged nitrogen. The imine was oriented in such a way that the hydrogen on the nitrogen was able to hydrogen bond to the negatively charged oxygen. This hydrogen bond stabilised the molecule and resulted in a relative energy of $3.0 \mathrm{kcal} \mathrm{mol}^{-\mathbf{1}}$.

\subsubsection{Rotational Barrier}

The rotational barrier for the 'O-deprotonation Pathway' was also calculated since the energies would differ due to the nitrogen being formally positively charged. Table 4.2 shows the energy of the cis-like conformer, the trans-like conformer, and the transition state separating the conformers for the $\left(\mathrm{S}_{\boldsymbol{p}}\right)$-FHPC containing diastereomer, along with the barrier for converting between the two. A scan of only the $\left(\mathrm{S}_{\boldsymbol{p}}\right)$-FHPC containing diastereomer was carried out since the energy of the diastereomers for the previous pathway only differed slightly. The same energy differences would therefore also be expected here.

The cis-like conformer was lower in energy than the trans-like conformer; 0.0 and $11.1 \mathrm{kcal} \mathrm{mol}^{-\mathbf{1}}$ respectively. The barrier for converting from the trans-like to the cis-like conformer was $23.4 \mathrm{kcal} \mathrm{mol}^{-\mathbf{1}}$, about eight times the size of the barrier 

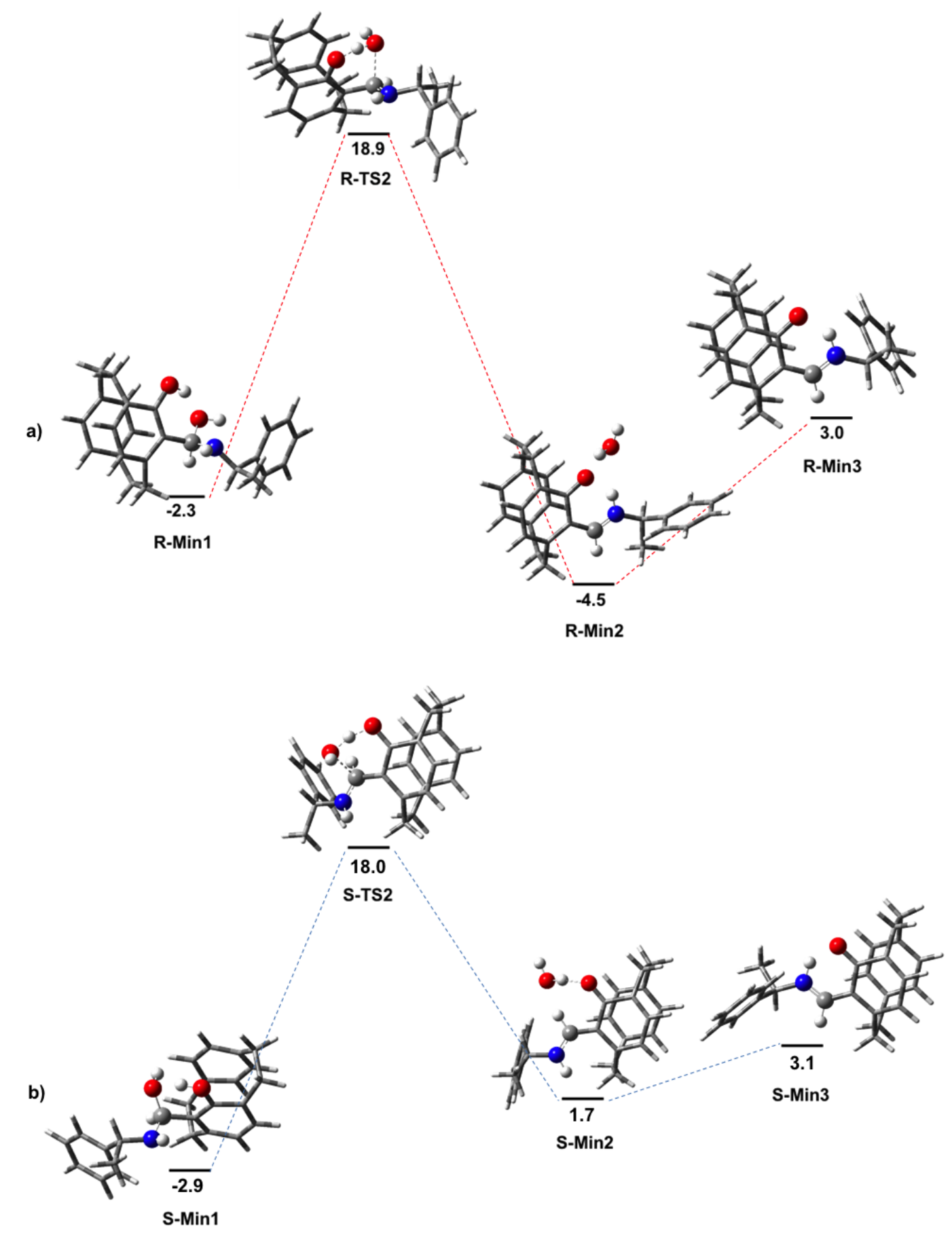

Figure 4.6: The structures of Min1, TS2, Min2 and Min3 for the $\left(\mathrm{R}_{p^{-}}\right.$ FHPC containing diastereomer a) and the ( $\mathrm{S}_{\boldsymbol{p}}$-FHPC containing diastereomer $\mathrm{b}$ ) are shown. The white, grey, red and blue atoms are $\mathrm{H}, \mathrm{C}, \mathrm{O}$, and $\mathrm{N}$ respectively. The electronic energies are with respect to the starting material, FHPC and (R)- $\boldsymbol{\alpha}$-PEAM, and are reported in $\mathrm{kcal} \mathrm{mol}^{-1}$. 


\begin{tabular}{llc}
\hline Intermediate & Orientation & Relative Energy $\left(\mathrm{kcal} \mathrm{mol}^{\mathbf{- 1}}\right)$ \\
\hline$\left(\mathrm{S}_{\boldsymbol{p}}\right)$-Min3 & cis -like & 0.0 \\
& trans-like & 11.1 \\
& TS & 34.5 \\
& Barrier $_{\text {trans } \rightarrow \text { cis }}$ & 23.4 \\
& Barrier $_{\text {cis } \rightarrow \text { trans }}$ & 34.5 \\
\hline
\end{tabular}

TABLE 4.2: The rotational barrier for converting from the cis-like conformation, imine rotated towards the hydroxyl group, to the trans-like conformation, imine rotated away from the hydroxyl group, was determined for the $\left(\mathrm{S}_{\boldsymbol{p}}\right)$-FHPC containing diastereomer. The dihedral angle with respect to the plane created by the two carbons of the cyclophane ring and the plane created by the carbon and nitrogen of the imine was varied.

found for the 'N-deprotonation Pathway'. The barrier for converting from the cis-like to the trans-like conformer was even higher at $34.5 \mathrm{kcal} \mathrm{mol}^{-\mathbf{1}}$. The cislike conformer was therefore the more stable conformation and rotation to afford the trans-like conformer would be unlikely due to the large barrer and the higher energy of the trans-like conformer.

\subsubsection{Transition State 3}

The final transition state (TS3, Figure 4.7) proceeded from Min3. Here the hydrogen between the oxygen and nitrogen migrated thus returning the cyclophane hydroxyl group. During this transition state the (R)- $\boldsymbol{\alpha}$-PEAM portion of the molecule remained in the same orientation as in Min3. This was most likely due to the hydrogen migrating over a very short distance. The cis-like conformation seen in Min3 created a direct trajectory for the hydrogen and thus the remainder of the molecule would not be affected. This close proximity accounts for the small energy barrier seen; about $4.0 \mathrm{kcal} \mathrm{mol}^{-\mathbf{1}}$. This is the smallest barrier apparent in this resolution method.

In the final product (Min4, Figure 4.7) the phenyl group had now rotated slighly away from the cyclophane and the reformed hydroxyl group. This will be to accomodate the hydrogen on the reformed hydroxyl group. This reformation stabilised the molecule by around $4.0 \mathrm{kcal} \mathrm{mol}{ }^{-1}$. 

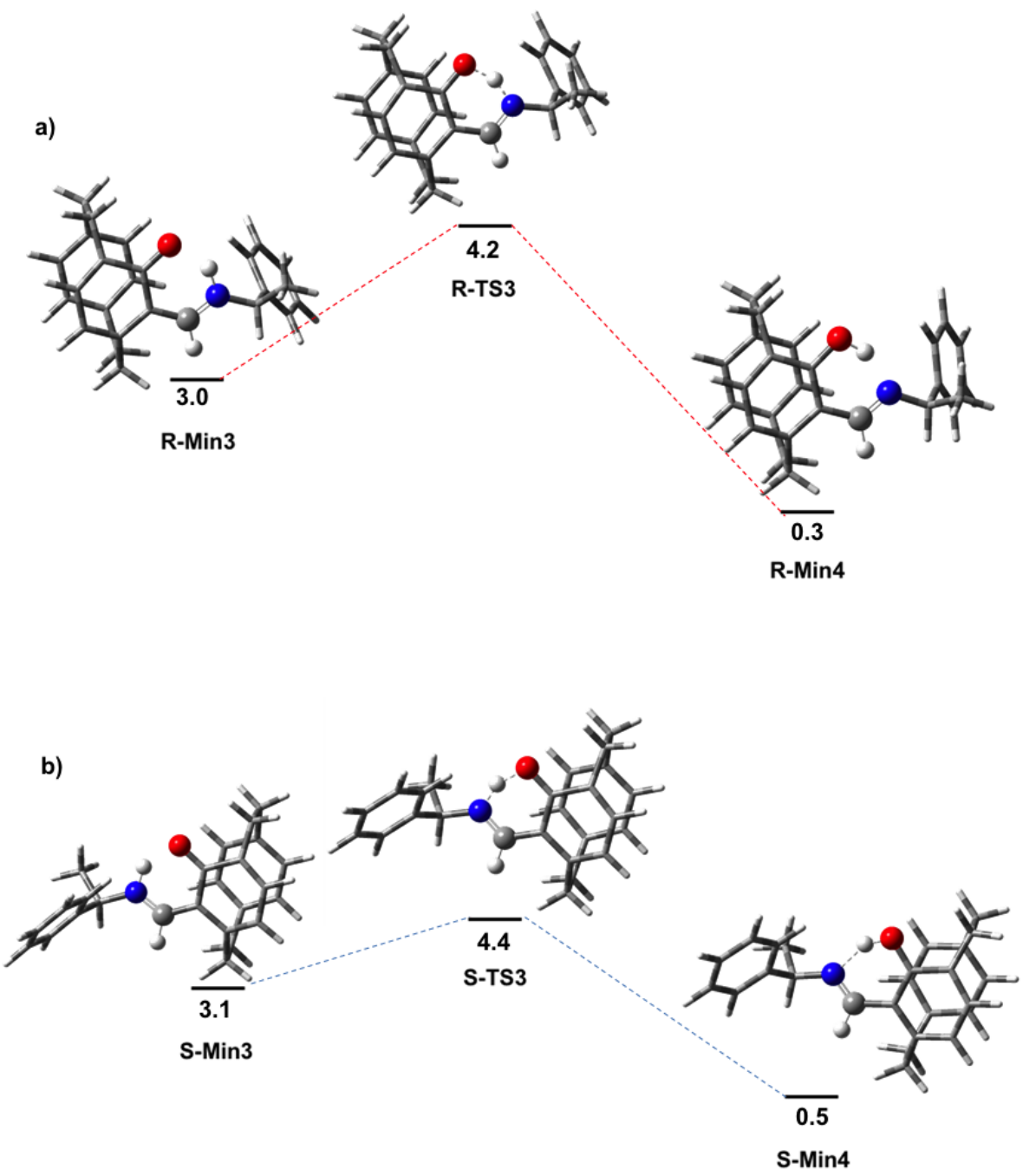

Figure 4.7: The structures of Min3, TS3, and Min4 for the $\left(\mathrm{R}_{p}\right.$-FHPC containing diastereomer a) and the $\left(\mathrm{S}_{\boldsymbol{p}}\right.$-FHPC containing diastereomer $\left.\mathrm{b}\right)$ are shown. The white, grey, red and blue atoms are $\mathrm{H}, \mathrm{C}, \mathrm{O}$, and $\mathrm{N}$ respectively. The electronic energies are with respect to the starting material, FHPC and (R)- $\boldsymbol{\alpha}$-PEAM, and are reported in $\mathrm{kcal} \mathrm{mol}^{-\mathbf{1}}$.

\subsection{Thermodynamics}

The Gibbs Free Energy, $\Delta \mathrm{G}$, was determined for the overall reaction to see how feasible the reaction for each enantiomer was. Table 4.3 shows the overall Gibbs Free Energy for each reaction. $\Delta \mathrm{G}$ was found to be 0.3 for the reaction containing $\left(\mathrm{R}_{\boldsymbol{p}}\right)$-FHPC and $0.4 \mathrm{kcal} \mathrm{mol}^{-\mathbf{1}}$ for the reaction containing $\left(\mathrm{S}_{\boldsymbol{p}}\right)$-FHPC. The values are both positive and differ by only $0.1 \mathrm{kcal} \mathrm{mol}^{-1}$. This positive energy indicates 
the reaction is nonspontaneous. Since the values are only slightly positive the reaction is still able to occur as long as energy is put into the reaction.

\begin{tabular}{lc}
\hline Enantiomer of FHPC & Overall $\boldsymbol{\Delta} \mathrm{G}\left(\mathrm{kcal} \mathrm{mol}^{\mathbf{- 1}}\right)$ \\
\hline $\mathrm{R}_{\boldsymbol{p}}$ & 0.3 \\
$\mathrm{~S}_{\boldsymbol{p}}$ & 0.4 \\
\hline
\end{tabular}

TABLE 4.3: The Gibbs Free Energies, $\Delta \mathrm{G}$, for the overall reaction are reported for both enantiomers.

\subsection{Discussion}

Both enantiomers of FHPC, $\left(\mathrm{S}_{\boldsymbol{p}}\right)$ and $\left(\mathrm{R}_{\boldsymbol{p}}\right)$, were reacted with one enantiomer of PEAM, (R)- $\boldsymbol{\alpha}$-PEAM. The reaction between these two enantiomers caused the formaton of diastereomers because the molecules were no longer mirror images. One chiral centre, the one from (R)- $\boldsymbol{\alpha}$-PEAM, was the same in both molecules whereas, contrariwise, the chirality of FHPC had been inverted. The formation of diastereomers was the desired result of this resolution method due to their differing energies. This allowed for their separation. If the energy difference between the diastereomers is large enough they are consequently able to be separated with relative ease. If, however, the energies are still too similar separation becomes difficult. A resolution method relying on the formation of diastereomers must therefore possess diastereomeric products with a relatively large energy difference.

A resolution method will be superior if the energy of the final product is significantly distinct. Combined with low energy barriers this will give a better separation and therefore a higher distereomeric excess $(d e)$. To determine which of the two pathways presented above was superior they will be discussed separetely before a comparison is done.

\subsubsection{N-deprotonation Pathway}

The first energy barrier for the $\left(R_{p}\right)$ and $\left(S_{p}\right)$ containing diastereomers were 26.7 and $26.5 \mathrm{kcal} \mathrm{mol}^{-\mathbf{1}}$ respectively. The energy difference, of $0.2 \mathrm{kcal} \mathrm{mol}^{-\mathbf{1}}$, was negligible. The energy differrence was too small to aid in the separation process although the transition state was diastereomeric because of the chiral centres present in the molecule. 
During this transition state, $(\mathrm{R})$ - $\boldsymbol{\alpha}$-PEAM approached both enantiomers of FHPC from below the cyclophane i.e. the plane of the most substituted ring which was not obstructed by the unsubstituted ring. This was as a result of the top face of the carbonyl being shielded due to the unsubstituted ring of the cyclophane. The cyclophane is very rigid and thus cannot rotate to accomodate the approaching amine if it were to attack from above the carbonyl. Throughout the transition state for the $\left(\mathrm{S}_{\boldsymbol{p}}\right)$-FHPC containing diastereomer the methyl group and the phenyl group of (R)- $\boldsymbol{\alpha}$-PEAM were oriented away from the cyclophane. The hydrogen on the nitrogen was therefore under the cyclophane ring. For the $\left(\mathrm{R}_{\boldsymbol{p}}\right)$-FHPC containing diastereomer the methyl group of (R)- $\boldsymbol{\alpha}$-PEAM was oriented under the ring of the cyclophane while the phenyl group was oriented away. The slight energy difference observed for this transition state can therefore be explained due to a bulkier group being closer to the cyclophane for the $\left(\mathrm{R}_{p}\right)$-FHPC containing diastereomer.

A new chiral centre was formed at the carbon of the carbonyl during this transition state. Since the amine attacked from below the cyclophane the configuration of this chiral centre matched the configuration of the cyclophane. The $(\mathrm{R})$-configuration was obtained for the $\left(\mathrm{R}_{\boldsymbol{p}}\right)$-FHPC containing diastereomer while for the $\left(\mathrm{S}_{\boldsymbol{p}}\right)$-FHPC containing diastereomer the configuration was $(\mathrm{S})$. This chiral centre was then later removed as a result of the lose of the hydroxyl group on the carbonyl carbon.

The resulting minimum, Min1, was more stable than the cyclophane. The $\left(\mathrm{R}_{\boldsymbol{p}}\right)$ FHPC containing diastereomer was slightly higher in energy at $-2.3 \mathrm{kcal} \mathrm{mol}^{-\mathbf{1}}$ than the $\left(\mathrm{S}_{\boldsymbol{p}}\right)$-FHPC containing diastereomer at $-2.9 \mathrm{kcal} \mathrm{mol}^{-\mathbf{1}}$. The $\left(\mathrm{S}_{\boldsymbol{p}}, \mathrm{R}, \mathrm{S}\right)$ diastereomer was further stabilised than the $\left(\mathrm{R}_{\boldsymbol{p}}, \mathrm{R}, \mathrm{R}\right)$-diastereomer to result in an energy difference of $0.6 \mathrm{kcal} \mathrm{mol}^{\mathbf{- 1}}$; four times the difference of TS1. This energy difference was, however, still very small and therefore separation could be difficult.

Both diastereomers were oriented so that the phenyl group of (R)- $\boldsymbol{\alpha}$-PEAM was almost coplanar with the bottom ring of the cyclophane. This orientation resulted in the greatest distance between the phenyl group and the cyclophane. The hydrogen on the nitrogen for the $\left(\mathrm{R}_{p}\right)$-FHPC containing diastereomer was in closer proximity to the hydrogen on the carbon of the former carbonyl than for the $\left(\mathrm{S}_{\boldsymbol{p}}\right)$ FHPC containing diastereomer. Here, the hydrogens were almost anti-periplanar. The closer proximity could explain the small energy difference observed. 
Due to the orientation of the newly formed hydroxyl group, hydrogen bonding was possible between this oxygen and the hydrogen of the cyclophane hydroxyl group, thus stabilising the molecule. The formation of a hydrogen bond explained the greater stability seen for these minima compared to FHPC.

For the next transition state, TS2, the barrier was nearly double the size of the previous transition state, TS1. The $\left(\mathrm{S}_{\boldsymbol{p}}\right)$-FHPC containing diastereomer was still lower in energy in comparison to the $\left(\mathrm{R}_{p}\right)$-FHPC containing diastereomer; 55.0 and $56.4 \mathrm{kcal} \mathrm{mol}^{-1}$ respectively. This energy difference of $1.4 \mathrm{kcal} \mathrm{mol}^{-\mathbf{1}}$ was larger still than for the previous two transition states. In spite of the increase the difference remained negligible.

The cyclophane hydroxyl group was oriented away from the forming imine which had adopted the trans-like conformation during the transition state. Hydrogen bonding was no longer possible between these two groups thereby explaining the large energy barrier. For the $\left(\mathrm{R}_{p}\right)$-FHPC containing diastereomer the $\mathrm{CH}_{3}$ group was facing up to ensure the phenyl group was oriented away from the forming water molecule. For the $\left(\mathrm{S}_{\boldsymbol{p}}\right)$ containing diastereomer the phenyl group was, however, facing up. The orientation adopted by this diastereomer was lower in energy which was most likely due to the hydroxyl group not beingt hindered during the deprotonation of the nitrogen, and consequent formation of water. For both diastereomers the water molecule leaves via the bottom of the cyclophane. The (R)- $\boldsymbol{\alpha}$-PEAM portion of the molecule was not very flexible because of the large phenyl group. The top face of the cyclophane was therefore too protected and resulted in the water molecule leaving below the cyclophane. The imine remained in the trans-like orientation and hydrogen bonding between the nitrogen and oxygen was not possible. During this transition state the chiral centre that had been formed in the first transition state was no longer present. This was because of the removal of the hydroxyl group and the consequent formation of the imine.

For Min2 the $\left(R_{p}, R\right)$-diastereomer was now lower in energy than the $\left(S_{p}, R\right)$ diastereomer; 5.9 and $7.8 \mathrm{kcal} \mathrm{mol}^{-1}$ respectively. For both diastereomers the cyclophane hydroxyl group was oriented away from the newly formed imine. This was probably because the current orientation allowed for hydrogen bonding between the nitrogen and the hydrogen of the water molecule. If the hydrogen on the hydroxyl group were oriented in towards the imine it would be in an unfavourbale position with respect to the hydrogen of the water molecule. Since the nitrogen 
only had one electron pair, in order to form a hydrogen bond, the hydrogen of the hydroxyl group must be oriented away.

The energy difference for the two diastereomers was $1.9 \mathrm{kcal} \mathrm{mol}^{\mathbf{- 1}}$, which was the largest energy difference apparent in this 'N-deprotonation Pathway'. The $\mathrm{CH}_{\mathbf{3}}$ group was oriented closer to the water molecule for the $\left(\mathrm{S}_{\boldsymbol{p}}\right)$-FHPC containing diastereomer. This was to endeavour to accomodate for the water molecule. For the $\left(\mathrm{R}_{p}\right)$-FHPC containing diastereomer the $\mathrm{CH}_{\mathbf{3}}$ group was below the cyclophane, which opened up space for the water molecule without a destabilising interaction with the $\mathrm{CH}_{\mathbf{3}}$ group. The $\left(\mathrm{S}_{\boldsymbol{p}}, \mathrm{R}\right)$-diastereomer was therefore higher in energy because of this interaction of the $\mathrm{CH}_{3}$ group and the water molecule.

For Min3 both stereoisomers were stabilised compared to the previous minimum. The $\left(\mathrm{R}_{\boldsymbol{p}}\right)$-FHPC containing diastereomer remained lower in energy than the $\left(\mathrm{S}_{\boldsymbol{p}}\right)$ FHPC containing diastereomer; 0.3 and $0.5 \mathrm{kcal} \mathrm{mol}^{-1}$ respectively. Here, the water molecule was removed resulting in the hydrogen on the hydroxyl group rotating in towards the imine. This allowed for hydrogen bonding between the nitrogen and the hydrogen of the hydroxyl group, and thus resulted in stabilising the diastereomers. The energy difference was much smaller than the difference for Min2; $0.2 \mathrm{kcal} \mathrm{mol}^{-\mathbf{1}}$. This lack of a significant difference was because of the orientation of the (R)- $\boldsymbol{\alpha}$-PEAM portion of the molecule. For both diastereomers the phenyl group was oriented away from the cyclophane. The $\mathrm{CH}_{\mathbf{3}}$ group was, however, oriented down below the cyclophane for the $\left(\mathrm{R}_{\boldsymbol{p}}\right)$ containing diastereomer compared to above for the $\left(\mathrm{S}_{\boldsymbol{p}}\right)$ containing diastereromer. The proximity of this methyl group thus slightly increased the energy of the $\left(\mathrm{S}_{\boldsymbol{p}}, \mathrm{R}\right)$-diastereomer.

The largest energy difference for this pathway was seen for Min2, $1.9 \mathrm{kcal} \mathrm{mol}^{-1}$. This energy difference was still very small but larger than the difference in Min3 and suggests that a better separation of the diastereomers could be achieved at Min2. The presence of the water resulted in the greatest energy difference because it was able to orient itself in different ways. Since the amine used for this resolution reaction was not very flexible there are only a small number of conformations possible during the reaction. The presence of the water molecule in Min3 thus provides more conformations and thereby there was a higher chance of a large energy difference.

For this resolution method to be successful using this mechanism, the diastereomers cannot be separated before the imine is formed. From these results the 
energy difference between the diastereomers could potentially be further increased through the use of an amine with more groups. The greater number of groups would allow for more conformational flexability. This should result in greater energy differences because of the possibility of one diastereomer being in a much more favourable conformation.

\subsubsection{O-deprotonation Pathway}

The first transition state (TS1), first minimum Min1 and final product was identical for this pathway as the 'N-deprotonation Pathway' as discussed above. This pathway deviated at the second transition state. Here, the hydroxyl group that was ejected during TS2 deprotonated the cyclophane hydroxyl group instead of the nitrogen. TS2 presented a barrier of about $20 \mathrm{kcal} \mathrm{mol}^{\mathbf{1}}$ for both diastereomers. The barrier for the $\left(\mathrm{R}_{\boldsymbol{p}}\right)$-FHPC containing diastereomer was greater by $0.9 \mathrm{kcal}$ $\mathrm{mol}^{-\mathbf{1}}$. Here, the water molecule was ejected and left adjacent to the cyclophane. The forming imine was close to the cis-like orientation thus the departing hydroxyl group was oriented slightly away from the cyclophane. This was possible because the phenyl group of (R)- $\boldsymbol{\alpha}$-PEAM had rotated to be below the cyclophane and the methyl group had been oriented away from the water molecule. This opened up space for the water molecule to leave via the top side of the forming imine. For the $\left(\mathrm{S}_{\boldsymbol{p}}\right)$-FHPC containing diastereomer the water molecule was ejected to the side and slightly below the cyclophane. The imine was oriented in the trans-like conformation for this to occur. The phenyl group was oriented to be adjacent to the cyclophane, while the methyl group was below the cyclophane. This allowed for the water molecule to leave in this sideways fashion. The slight energy difference can be explained by the water molecule having been ejected adjacent to the cyclophane for the $(\mathrm{R} p, \mathrm{R})$-diastereomer.

The resulting minimum, Min2, had an energy difference of $4.2 \mathrm{kcal} \mathrm{mol}^{\mathbf{1}}$ with the $\left(\mathrm{S}_{\boldsymbol{p}}, \mathrm{R}\right)$-diastereomer remaining more stabilised than the $\left(\mathrm{R}_{\boldsymbol{p}}, \mathrm{R}\right)$-diastereomer; 1.7 and $5.9 \mathrm{kcal} \mathrm{mol}^{-\mathbf{1}}$ respectively. This was the largest energy difference seen for this pathway. Both diastereomers are stabilised by a hydrogen bond between the hydrogen of the water molecule and the oxygen of the cyclophane. The imine in the $\left(\mathrm{R}_{\boldsymbol{p}}\right)$-FHPC containing diastereomer had rotated in towards the oxygen thus adopting the cis-like conformation. Since this conformation was lower in energy than the trans-like conformation seen for the $\left(\mathrm{S}_{\boldsymbol{p}}\right)$-FHPC containing diastereomer, 
it must be more stable. The orientation of the methyl and phenyl group would also contribute to the overall stability of the molecule. For the $\left(R_{p}, R\right)$-diastereomer the phenyl and methyl group are nearly coplanar with the imine. This orientation generated space for the water molecule. For the $\left(\mathrm{S}_{\boldsymbol{p}}, \mathrm{R}\right)$-diastereomer the phenyl group was oriented up and adjacent to the cyclophane. This brought it in relatively close proximity to the interacting water molecule thus raising the energy of the molecule.

The minimum without the water, Min3, resulted in the $\left(\mathrm{S}_{\boldsymbol{p}}\right)$-FHPC containing diastereomer being destabilisied while the $\left(\mathrm{R}_{\boldsymbol{p}}\right)$-FHPC containing diastereomer was stabilised. The $\left(\mathrm{R}_{\boldsymbol{p}}, \mathrm{R}\right)$-diastereomer was now lower in energy by $0.1 \mathrm{kcal} \mathrm{mol}^{-\mathbf{1}}$ . The $\left(\mathrm{S}_{\boldsymbol{p}}\right)$-FHPC containing diastereomer had an energy of $3.1 \mathrm{kcal} \mathrm{mol}^{-\mathbf{1}}$ while the $\left(\mathrm{R}_{\boldsymbol{p}}\right)$-FHPC containing diastereomer had an energy of $3.0 \mathrm{kcal} \mathrm{mol}^{-\mathbf{1}}$.

For the $\left(\mathrm{R}_{\boldsymbol{p}}, \mathrm{R}\right)$-diastereomer the $\mathrm{CH}_{3}$ group of $(\mathrm{R})$ - $\boldsymbol{\alpha}$-PEAM had rotated so that it was below the cyclophane. For the other diastereomer the imine was now in the cis-like conformation, thus allowing for the formation of a hydrogen bond between the hydrogen of the nitrogen and the oxygen of the cyclophane. This hydrogen bonding was also present for the $\left(\mathrm{R}_{\boldsymbol{p}}\right)$-FHPC containing diastereomer and thus both diastereomers are stabilised through hydrogen bonding. The energy of the $\left(\mathrm{S}_{\boldsymbol{p}}\right)$-FHPC containing diastereomer increased in energy when the water molecule was removed. This energy increase of $0.4 \mathrm{kcal} \mathrm{mol}^{-1}$ was most likely due to the hydrogen bond between the water molecule and oxygen was broken combined with the change in orientation of the methyl and phenyl group.

The cis-like orientation adopted in Min3 allowed for the migration of the hydrogen from the nitrogen to the oxygen of the carbonyl, TS3. This energy barrier was found to be $1.3 \mathrm{kcal} \mathrm{mol}^{-1}$. This low barrier was due to the orientation adopted in the previous minimum. The $\left(\mathrm{R}_{\boldsymbol{p}}\right)$-FHPC containing diastereomer remained lower in energy compared to the $\left(\mathrm{S}_{\boldsymbol{p}}\right)$-FHPC diastereomer. No change in orientation occured for either diastereomer because of the close proximity of the hydrogen to the oxygen of the cyclophane. The resulting product, Min4, was then lower in energy than Min3 due to the reformation of the hydroxyl group and consequent neutralisation of the formal charges. The same product was formed here as in the 'N-deprotonation Pathway'.

For this mechanism the largest energy difference was found for Min2, where the water molecule was still present. This difference in energy could therefore improve 
the resolution method if separation were to occur here. The removal of the water molecule reduces the energy difference from 4.2 to $0.1 \mathrm{kcal} \mathrm{mol}^{-\mathbf{1}}$. The difficulty with separation during Min2 is the fact that the water molecule was responsible for this energy difference. If the distance between the water molecule and the cyclophane molecule were increased the energies of both diastereomers would start to approach that of Min3 where the energy difference was now small.

These structures and energies were determined in the gas phase. The involvement of any solvent was therefore ignored. This resolution method proposed by Antanov et al. was carried out in benene and ethanol in a 3.8:1 ratio. ${ }^{39}$ The ethanol present in the solution could provide a large enough energy separation in Min3 when the water molecule was no longer present. The issue with this is that the following transition state, TS3, was low in energy therefore the final product will form unless the reaction temperature were kept low. This, in turn, causes the first transition state not to be accessible.

An alternative approach to improve on this resolution method would be to use a different amine. The energy differences for this method are typically quite small and this was probably due to the few conformations that can be adopted by the molecule due to the rigid cyclophane backbone and the small amine. The use of a longer amine with repelling groups such as a carbonyl, amide, or carboxylic acid could stabilise one diastereomer while destabilising the other.

\subsubsection{Comparison}

Both mechanisms must go through TS1 and to Min1 before they start to deviate. The barrier for TS1 was relatively low meaning that the reaction should occur reasonably quickly. It was the second energy barrier that was extremely different. For the 'N-deprotonation Pathway' the energy barrier was roughly about 55.0 kcal mol ${ }^{-1}$, while for the alternative mechanism the barrier was only about 18.0 kcal $\mathrm{mol}^{-1}$. That energy barrier was more than half the size of that of the ' $\mathrm{N}$ deprotonation Pathway'. The reaction will proceed via the transition state that has a lower energy barrier. This means that the transition state that was more likley to occur will be the one from the 'O-deprotonation Pathway'. The departing hydroxyl group will therefore deprotonate the hydroxyl group on the cyclophane to give the resulting water molecule. This is true for both enantiomers of FHPC. 
Min2 was also lower in energy for the 'O-deprotonation Pathway' which means that there was a driving force for this reaction to occur other than it just being the lower energy barrier. For the next minimum, where the water molecule was removed, the $\left(\mathrm{S}_{\boldsymbol{p}}\right)$-FHPC containing diastereomer was destabilised while the $\left(\mathrm{R}_{\boldsymbol{p}}\right)$ FHPC containing diastereomer was stabilised. This means that the $\left(\mathrm{R}_{p}\right)$-FHPC containing diastereomer will easily drop to that minimum, while the $\left(\mathrm{S}_{\boldsymbol{p}}\right)$-FHPC containing diastereomer required a small amount of energy to reach that minimum; $1.5 \mathrm{kcal} \mathrm{mol}^{\mathbf{- 1}}$. The final transition state, TS3, had a very low energy barrier and therefore the formation of the product can occur quickly and smoothly to give the same products as the other pathway. This does mean that the 'O-deprotonation Pathway' was preferred both kinetically and thermodynamically.

Fortunately the 'O-deprotonation Pathway' also provided greater energy differences; 4.2 compared to $1.9 \mathrm{kcal} \mathrm{mol}^{-\mathbf{1}}$. There was therefore the possibility of stopping the reaction earlier where there was a greater energy difference between the diastereomers. In this resolution method proposed by Antanov et al. the reaction was carried out in benzene/ethanol and was refluxed for 3 hours. ${ }^{39}$ Since the reaction was refluxed the 'N-deprotonation Pathway' becomes accessible. This in combination with the 3 hours, allows for the more thermodynamic product to form. The reaction would therefore still proceed via the 'O-deprotonation Pathway'. There is, however, an issue with stopping the reaction at an earlier point since removal of the water molecule caused a considerable drop in the energy difference. If the reaction were carried out in the actual solvent, the ethanol could hydrogen bond with the oxygen in Min2 and therefore the energy difference could remain large. The increased size of the molecule could prevent this interaction but it could also cause an even larger energy difference.

This could also then be true for the final minimum, Min4. The energy difference seen was very small and could account for the $36 \%$ de obtained with respect to the $\left(R_{p}, R\right)$-diastereomer. The resolution method should therefore be able to be improved through the use of a longer amine which can adopt more conformations.

The rate determining step for the 'N-deprotonation Pathway' is TS2 while for the 'O-deprotonation Pathway' it is TS1 because it had the largest energy barrier. 


\subsubsection{Thermodynamics}

$\Delta \mathrm{G}$ for the overall reaction was found to be positive for both enantiomers of FHPC at room temperature. The reaction is therefore nonspontaneous when carried out in the gas phase meaning energy would be required for this reaction to occur when carried out at room temperature. The actual reaction carried out by Antonov et al. was refluxed for three hours and did proceed. ${ }^{39}$ The extra energy provided by heating the solution could have been why the reaction occured.

The energy difference between the diastereomers remained small even when thermodynamics was taken into consideration. Once again separation of the diastereomers at the end of the reaction would be difficult. 


\section{Chapter 5}

\section{Comparison of Resolution Reactions}

The two resolution methods of FHPC discussed in the previous two chapters involved the use of an amine to form diastereomeric imines. Two pathways, 'Ndeprotonation pathway' and 'O-deprotonation pathway', were considered and the most suitable pathway was determined for each resolution method. Here, the different resolution methods will be compared to obtain further insight into how these two methods operate.

\subsection{N-Deprotonation Pathway}

The energy differences were, in general, much larger for the first method than for the second. For a resolution method to be successful there must be an energy difference between the two diastereomers. The larger this energy difference is the easier it is to separate the two diastereomers. The first method, in general, involved energy differences that were much larger. It would, therefore, be more successful. This was, however, not in agreement with the experimental work carried out by Rozenberg et al. and Antanov et al. ${ }^{28,39}$

A new resolution method was proposed by Antanov et al. to try and improve on the selectivity observed in the first method through the use of a simpler resolution method. ${ }^{39}$ The work carried out here indicated that the first method was not successful because of the use of copper(II) perchlorate. The coordination of the 
imine to the copper greatly increased the energy for both diastereomers. This increase was, however, associated with a decrease in the energy difference between the diastereomers; $0.1 \mathrm{kcal} \mathrm{mol}^{\mathbf{- 1}}$ versus $0.6 \mathrm{kcal} \mathrm{mol}^{\mathbf{- 1}}$ in Min3. This decrease in energy when the diastereomers coordinate to copper could be why a new resolution method was required. If the first resolution method were used without copper(II) perchlorate the separation should become easier and thereby increase the de and ee observed.

The transition states and intermediates found for both resolution methods are the same with respect to the process occuring. The variation in the energy differences between the diastereomers in the two methods was therefore due to the amine used. (S)-valyl-(S)-valine was employed for the first resolution method whereas (R)- $\boldsymbol{\alpha}$-PEAM was utilised in the second.

The barrier of the first transition state was reasonably large for both methods. The energy difference for the first method was much larger than for the second. The $\left(\mathrm{S}_{\boldsymbol{p}}\right)$-FHPC containing diastereomer was higher in energy for the first resolution method, but lower for the second resolution method. This was most likely due to the amine in the second resolution method attacking the cyclophane so that the $\mathrm{CH}_{3}$ group was below the plane of the substituted ring of the cyclophane, with respect to the unsubstituted ring, instead of the hydrogen; as seen for the $\left(\mathrm{S}_{\boldsymbol{p}}\right)$ FHPC containing diastereomer. For the first resolution method the amine attacked both enantiomers of FHPC in the same way. The hydrogen was oriented below the substituted ring instead of the bulkier carbon chain of (S)-valyl-(S)-valine. This orientation reduced sterics and thus lowered the energy of the system.

The resulting minimum Min1 was the lowest energy minimum for both methods. It was lower in energy than FHPC, except for S-Min1 for the first resolution method. The reason this minimum was higher in energy than the $\left(\mathrm{R}_{p}\right)$-FHPC containing diastereomer was because of increased steric interactions due to part of the (S)-valyl-(S)-valine portion of the molecule being below both rings of the cyclophane. This consequently raised the energy above that of FHPC.

The second transition state was the largest barrier for this pathway for both methods. The energy difference was larger for the second resolution method. For all transition states the water molecule was ejected below the substituted ring of the cyclophane with respect to the unsubstituted ring. The forming imine was rotated away from the cyclophane hydroxyl group for both diastereomers in both 
the methods. The $\left(\mathrm{R}_{\boldsymbol{p}}\right)$-FHPC containing diastereomer was lower in energy for the first method but higher in the second resolution method. This was because the amine portion of the molecule was furthest away in TS2 by adopting a flatter orientation.

Min2 was higher in energy than the previous minimum Min1 for both methods. For the first resolution method the $\left(\mathrm{R}_{\boldsymbol{p}}\right)$-FHPC containing diastereomer was higher in energy but, in comparison, it was lower in energy for the second resolution method. This diastereomer was higher in energy for the first resolution method due to an extra hydrogen bond between the water molecule and the nitrogen of the amide in (S)-valyl-(S)-valine. The formation of this hydrogen bond decreased the distance between the amide containing portion and the cyclophane, thus raising the energy. For the second resolution method the (R)- $\boldsymbol{\alpha}$-PEAM portion was always oriented away from the cyclophane and ergo both energies are much closer.

The removal of the water molecule, Min3, caused a decrease in the energy difference between the diastereomers for both methods. The energy of the $\left(\mathrm{R}_{\boldsymbol{p}}\right)$-FHPC containing diastereomer in the first resolution method was now lower in energy than the $\left(\mathrm{S}_{\boldsymbol{p}}\right)$-FHPC containing diastereomer. This energy switch did not occur in the second resolution method. All diastereomers contained a hydrogen bond between the hydrogen of the oxygen and the nitrogen of the imine.

The largest energy difference for both methods was seen during Min2 where the water molecule hydrogen bonded with the stereoisomers. In the actual reaction carried out by Rozenberg et al. and Antanov et.al. the presence of the solvent would play a similar role to the water molecule. ${ }^{28,39}$ The energy differences that occured during the reaction could therefore be much larger since the presence of the water molecule in the mechanism found here caused a larger energy difference.

For the second resolution method, where (R)- $\boldsymbol{\alpha}$-PEAM was used instead, the water molecule that was formed during TS2 was ejected via the bottom of the cyclophane. This could be due to the (R)- $\boldsymbol{\alpha}$-PEAM group not being as flexible as the (S)-valyl-(S)-valine portion of the molecule. Thus in the second resolution method the water cannot leave via the top of the cyclophane since there was no orientation of the (R)- $\boldsymbol{\alpha}$-PEAM portion that allowed for this.

The energy difference between the diastereomers could be increased by utilising an amine that is between the two used here with respect to size. The large amine, (S)-valyl-(S)-valine, was too flexible and therefore had too many conformations 
which meant every step of the mechanism could be stabilised. (R)- $\boldsymbol{\alpha}$-PEAM did not provide, however, enough of a barrier for the reaction since the energies were too similar. An amine where the chain length is kept reasonably short, but larger groups are used that could stabilise the molecule via hydrogen bonding, would be ideal. The amine cannot, however, be too flexible. Great flexibility allows the molecule to adopt a vast number of conformations. This, in turn, decreases the possibility of large energy differences because the molecule is able to rotate to prevent a destabilising interaction due to the close proximity of groups.

\subsection{O-Deprotonation Pathway}

The 'O-deprotonation pathway' differs at the second transition state. The first transition state and minimum for this pathway, TS1 and Min1, are therefore the same. The first transition state was still the largest barrier for this pathway for both methods.

The second transition state for both resolution methods was a much lower energy barrier than that of TS1. This was because the hydrogen used in the migration was now close to the forming water molecule, whereas for the first transition state two separate molecules had to come together in the right orientation. The energy difference for the second resolution method was greater than for the first resolution method. The water molecule leaves via the bottom of the cyclophane for both diastereomers containing the $\left(\mathrm{S}_{\boldsymbol{p}}\right)$-FHPC, but not for the $\left(\mathrm{R}_{\boldsymbol{p}}\right)$-FHPC containing diastereomers.

In Min2 the energy difference was much larger for both methods. This was the largest energy difference seen in the entire pathway for both methods. The energy difference was greater for the first resolution method than the first. The $\left(\mathrm{R}_{\boldsymbol{p}}\right)$-FHPC containing diastereomer was higher in energy for the second method, whereas the $\left(\mathrm{S}_{\boldsymbol{p}}\right)$-FHPC containing diastereomer was higher in energy for the first method. For the first resolution method the $\left(\mathrm{R}_{p}\right)$-FHPC containing diastereomer was much lower in energy than the $\left(\mathrm{S}_{\boldsymbol{p}}\right)$-FHPC containing diastereomer. This large energy difference was due to the extra hydrogen bonding that was possible in the molecule. All diastereomers contained a hydrogen bond between the water molecule and the formally negative charged oxygen of the cyclophane. The $\left(\mathrm{R}_{p}\right)$-FHPC containing diastereomer for the first resolution method also contained 
two additional hydrogen bonds; between the oxygen of the water molecule and the hydrogen of the nitrogen of the amide, and between the hydrogen on the imine and the formally negative oxygen of the cyclophane. This greatly stabilised the molecule and thus lowered the energy of this diastereomer considerably with respect to the $\left(\mathrm{S}_{\boldsymbol{p}}\right)$-FHPC containing diastereomer. This was not present in the other resolution method and hence the energy difference was much smaller.

For both pathways the stability of the molecules during Min3 inverted. For the first method the $\left(\mathrm{R}_{\boldsymbol{p}}\right)$-FHPC containing diastereomer was greatly destabilised, whereas it was stabilised in the second method. The energy difference dropped for both pathways but to a much greater extent for the second resolution method. The energy difference was now negligible. All diastereomers contained a hydrogen bond between the hydrogen of the imine and the formally negative oxygen. This thereby stabilised the molecule.

The barrier of the final transition state, TS3, was approximately the same for both methods. The energy difference for the first resolution method remained relatively large while it was further decreased for the second method. The orientation of the diastereomers remained the same due to the short distance of migration.

The final minimum in which the imine had formed and the hydroxyl group was reformed was stabilised to a greater extent than Min3 for both methods. For the first resolution method the stability of the diastereomers were reversed once again so that the $\left(\mathrm{R}_{\boldsymbol{p}}\right)$-FHPC containing diastereomer was lower in energy just as it was in the second method; in this second method the stability of the diastereomers was not reversed.

The energy difference of Min4 for the second method was identical to that of the transition state, TS3; $0.2 \mathrm{kcal} \mathrm{mol}^{-1}$. For the first resolution method the difference was now much smaller than it was previously but was still larger than for the second method; $0.6 \mathrm{kcal} \mathrm{mol}^{-1}$. The first resolution method for this pathway should therefore also provide a greater energy difference and thereby make separaton easier and more successful.

For the second resolution method this pathway was preferred kinetically and thermodynamically due to the low energy transition state, TS2, and minimum, Min2. Min2 for the $\left(\mathrm{R}_{\boldsymbol{p}}\right)$-FHPC containing diastereomer was also thermodynamically preferred because it was lower in energy for this pathway. This was not the 
case for the $\left(\mathrm{S}_{\boldsymbol{p}}\right)$-FHPC containing diastereomer where the product from the ' $\mathrm{N}$ deprotonation pathway' gives the thermodynamic product.

Kinetic control for the first resolution method will therefore result in the 'Odeprotonation pathway'. On the other hand thermodynamic control will result in the $\left(\mathrm{R}_{p}\right)$-FHPC containing diastereomer to go through the 'O-deprotonation pathway', whereas the $\left(\mathrm{S}_{\boldsymbol{p}}\right)$-FHPC containing diastereomer will go through the 'N-deprotonation pathway'. This was because the resulting minimum was lower in energy. 


\section{Chapter 6}

\section{Conclusions}

The two methods for resolving racemic FHPC have been investigated here. The transition states where all found to be concerted processes. This was most likely to prevent the formation of formal charges since the calculations were carried out in the gas phase. As such there was no solvent present to stabilise a charged molecule. For both methods two possible pathways were found, 'N-deprotonation Pathway' and 'O-deprotonation Pathway'. It was found that the 'O-deprotonation Pathway' was more likely to occur for both resolution methods due to the second transition state, which differentiated the two methods, was lower for both enantiomers of FHPC when the hydroxyl was deprotonated instead of the nitrogen. This is because TS1 and Min1 where identical for both pathways and therefore TS2 was the step that determed which pathway was most likely to occur. The use of a smaller amine, (R)- $\boldsymbol{\alpha}$-PEAM vs (S)-valyl-(S)-valine, typically resulted in lower energies. This decrease in the overall energy, however, also caused a decrease in the energy difference between the diastereomers. The separation of these diastereomers is therefore much harder. Thus the original resolution method utilising (S)-valyl-(S)-valine should provide a better separation between the two diastereomers. The formation of the diastereomeric copper complexes however greatly decreases the usefulness of the method. The energy difference drops considerably and thus makes separation more difficult. If the copper were to be removed from the reaction entirely the separation should be much more successful.

The first transition state for the first resolution method was much lower in energy when the starting material was not coordinated to the copper. The reaction would 
therefore proceed through the 'Non-Coordinating Pathway' and thus making the copper obsolete.

The effect of different solvents on the energy of the copper complexes was determined to see whether a different solvent could stabilise the complexes. A stabilisation was seen for all solvents but ethanol stabilised the copper complexes to the greatest extent. 2-propanol, the solvent used experimentally, was found to only slightly destabilise the complexes when compared to ethanol. The energy difference of the diastereomeric copper complexes was still found to be extremely small. Separation of these complexes would therefore still be difficult and would result in low purity.

The 'O-deprotonation Pathway' was found to be preferred kinetically due to the low energy barrier of TS2 and also thermodynamically due to the greatly stabilised subsequent minimum, Min2. The most likely pathway for both resolution methods would therefore be the 'O-deprotonation Pathway'.

The energy difference between the two diastereomers of the imine was still small. Thus if the reaction could be controlled kinetically the diastereomers could be resolved at an earlier point thus increasing the purity. The resolution of racemic FHPC could be improved through the use of a smaller amine than (S)-valyl-(S)valine but with more possible conformations than $(\mathrm{R})$ - $\boldsymbol{\alpha}$-PEAM. This way one diastereomer would ideally be stabilised while the other is destabilised, thus increasing the energy difference between the diastereomers and improving upon the separation. 


\section{Bibliography}

[1] Moss, G. P. Pure Appl. Chem. 1996, 68, 2193-2222.

[2] Hopmann, K. H. Int. J. Quantum Chem. 2015, 1-18.

[3] Ghislieri, D.; Turner, N. J. Top. Catal. 2014, 57, 284-300.

[4] Todd, M. H. Separation of Enantiomers: Synthetic Methods; John Wiley \& Sons, 2014.

[5] Morrison, J. Asymmetric synthesis; Elsevier, 2012; Vol. 3.

[6] Seebach, D.; Oei, H. A. Angew. Chem. Int. Ed. 1975, 14, 634-636.

[7] Baudequin, C.; Brégeon, D.; Levillain, J.; Guillen, F.; Plaquevent, J. C.; Gaumont, A. C. Tetrahedron: Asymmetry 2005, 16, 3921-3945.

[8] Suzuki, Y.; Wakatsuki, J.; Tsubaki, M.; Sato, M. Tetrahedron 2013, 69, 9690-9700.

[9] Thormeier, S.; Carboni, B.; Kaufmann, D. E. J. Organomet. Chem. 2002, $657,136-145$.

[10] Lebel, H.; Trudel, C.; Spitz, C. Chem. Commun. 2012, 48, 7799.

[11] Sorochinsky, A. E.; Ueki, H.; Aceña, J. L.; Ellis, T. K.; Moriwaki, H.; Sato, T.; Soloshonok, V. a. Org Biomol. Chem. 2013, 11, 4503-7.

[12] Kragl, U.; Dwars, T. Trends Biotechnol. 2001, 19, 442-449.

[13] Wang, J. Tetrahedron Lett. 2013, 54, 5956-5959.

[14] Morales, M. R.; Mellem, K. T.; Myers, A. G. Angew. Chem. Int. Ed. 2012, $51,4568-4571$.

[15] Heravi, M. M.; Zadsirjan, V. Tetrahedron: Asymmetry 2013, 24, 1149-1188. 
[16] Khatik, G. L.; Sharma, R.; Kumar, V.; Chouhan, M.; Nair, V. a. Tetrahedron Lett. 2013, 54, 5991-5993.

[17] Pal, A.; Peng, Z.; Schuber, P. T.; Bhanu Prasad, B. a.; Bornmann, W. G. Tetrahedron Lett. 2013, 54, 5555-5557.

[18] Class, G. O. F.; Compounds, O. F. O. Pure Appl. Chem. 1995, 67, 13071375 .

[19] Brown, C. J.; Farthing, A. C. Nature 1949, 164, 915-916.

[20] Cram, D. J.; Steinberg, H. J. Am. Chem. Soc. 1951, 73, 5691-5704.

[21] Gibson, S. E.; Knight, J. D. Org. Biomol. Chem. 2003, 1, 1256-1269.

[22] Hopf, H. Tetrahedron 2008, 64, 11504-11516.

[23] Zhao, L.; Ma, Y.; He, F.; Duan, W.; Chen, J.; Song, C. J. Org. Chem. 2013, 78, 1677-1681.

[24] Dahmen, S.; Bräse, S. Isr. J. Chem. 2012, 52, 139-142.

[25] Marrocchi, A.; Tomasi, I.; Vaccaro, L. Isr. J. Chem. 2012, 52, 42-52.

[26] Mutoh, K.; Nakano, E.; Abe, J. J. Phys. Chem. A 2012, 116, 6792-6797.

[27] Clément, S.; Goudreault, T.; Bellows, D.; Fortin, D.; Guyard, L.; Knorr, M.; Harvey, P. D. Chem. Commun. 2012, 48, 8640.

[28] Rozenberg, V.; Kharitonov, V.; Antonov, D.; Sergeeva, E.; Aleshkin, A.; Ikonnikov, N.; Orlova, S.; Belokon', Y. Angew. Chem. Int. Ed. 1994, 33, 91-92.

[29] Tanji, S.; Ohno, A.; Sato, I.; Soai, K. Org. Lett. 2001, 3, 287-289.

[30] Rowlands, G. J. Isr. J. Chem. 2012, 52, 60-75.

[31] Duan, W.; Ma, Y.; He, F.; Zhao, L.; Chen, J.; Song, C. Tetrahedron: Asymmetry 2013, 24, 241-248.

[32] Cipiciani, A.; Fringuelli, F.; Piermatti, O.; Pizzo, F.; Ruzziconi, R. J. Org. Chem. 2002, 67, 2665-2670.

[33] Lu, Y.; Ma, Y.; Yang, S.; Ma, M.; Chu, H.; Song, C. Tetrahedron: Asymmetry 2013, 24, 1082-1088. 
[34] Zhao, L.; Ma, Y.; Duan, W.; He, F.; Chen, J.; Song, C. Org. Lett. 2012, 14, $5780-5783$.

[35] Vorontsova, N. V.; Bystrova, G. S.; Antonov, D. Y.; Vologzhanina, A. V.; Godovikov, I. a.; Il'in, M. M. Tetrahedron: Asymmetry 2010, 21, 731-738.

[36] Xin, D.; Ma, Y.; He, F. Tetrahedron: Asymmetry 2010, 21, 333-338.

[37] Bolm, C.; Whelligan, D. K. Adv. Synth. Catal. 2006, 348, 2093-2100.

[38] He, F.; Ma, Y.; Zhao, L.; Duan, W.; Chen, J.; Zhao, Z. Tetrahedron: Asymmetry 2012, 23, 809-817.

[39] Antonov, D. Y.; Belokon, Y. N.; Ikonnikov, N. S.; Orlova, S. a.; Pisarevsky, A. P.; Raevski, N. I.; Rozenberg, V. I.; Sergeeva, E. V.; Struchkov, Y. T.; Tararov, V. I.; Vorontsov, E. V. J. Chem. Soc. Perk. T. 1 1995, 1873 .

[40] Wang, D.; Ma, Y.; He, F.; Duan, W.; Zhao, L.; Song, C. Synthetic Commun. 2013, 43, 810-825.

[41] Kitagaki, S.; Ohta, Y.; Tomonaga, S.; Takahashi, R.; Mukai, C. Tetrahedron: Asymmetry 2011, 22, 986-991.

[42] Hong, B.; Ma, Y.; Zhao, L.; Duan, W.; He, F.; Song, C. Tetrahedron: Asymmetry 2011, 22, 1055-1062.

[43] Meyer-Eppler, G.; Vogelsang, E.; Benkhäuser, C.; Schneider, A.; Schnakenburg, G.; Lützen, A. Eur. J. Org. Chem. 2013, 4523-4532.

[44] Morisaki, Y.; Hifumi, R.; Lin, L.; Inoshita, K.; Chujo, Y. Polym. Chem. 2012, 3, 2727.

[45] Friedmann, C. J.; Ay, S.; Bräse, S. J. Org. Chem. 2010, 75, 4612-4614.

[46] Vorontsova, N. V.; Rozenberg, V. I.; Sergeeva, E. V.; Vorontsov, E. V.; Starikova, Z. a.; Lyssenko, K. a.; Hopf, H. Chem.-Eur. J. 2008, 14, 46004617.

[47] Kitagaki, S.; Ueda, T.; Mukai, C. Chem. Commun. 2013, 49, 4030-2.

[48] David, O. R. P. Tetrahedron 2012, 68, 8977-8993. 
[49] Qu, B.; Ma, Y.; Ma, Q.; Liu, X.; He, F.; Song, C. J. Org. Chem. 2009, 74, 6867-6869.

[50] Kreis, M.; Friedmann, C. J.; Bräse, S. Chem.-Eur J. 2005, 11, 7387-7394.

[51] Zhang, T. Z.; Dai, L. X.; Hou, X. L. Tetrahedron: Asymmetry 2007, 18, $251-259$.

[52] Duan, W.; Ma, Y.; Qu, B.; Zhao, L.; Chen, J.; Song, C. Tetrahedron: Asymmetry 2012, 23, 1369-1375.

[53] Lennartz, P.; Raabe, G.; Bolm, C. Isr J. Chem. 2012, 52, 171-179.

[54] Masterson, D. S.; Shirley, C.; Glatzhofer, D. T. J. Mol. Catal. A-Chem 2012, 361-362, 111-115.

[55] Frank, D.; Nieger, M.; Friedmann, C.; Lahann, J.; Bräse, S. Isr. J. Chem. 2012, 52, 143-148.

[56] Danilova, T. I.; Rozenberg, V. I.; Starikova, Z. a.; Bräse, S. Tetrahedron: Asymmetry 2004, 15, 223-229.

[57] Clément, S.; Guyard, L.; Knorr, M.; Dilsky, S.; Strohmann, C.; Arroyo, M. J. Organomet. Chem. 2007, 692, 839-850.

[58] Duan, W.; Ma, Y.; Xia, H.; Liu, X. J. Org. Chem. 2008, 73, 4330-4333.

[59] Jiang, B.; Lei, Y.; Zhao, X.-L. J. Org. Chem. 2008, 73, 7833-7836.

[60] Zhuravsky, R.; Starikova, Z.; Vorontsov, E.; Rozenberg, V. Tetrahedron: Asymmetry 2008, 19, 216-222.

[61] Sugiyama, S.; Aoki, Y.; Ishii, K. Tetrahedron: Asymmetry 2006, 17, 28472856 .

[62] Lauterwasser, F.; Vanderheiden, S.; Bräse, S. Adv. Synth. Catal. 2006, 348, 443-448.

[63] Kreis, M.; Nieger, M.; Bräse, S. J. of Organomet. Chem. 2006, 691, 21712181.

[64] Zhuravsky, R. P.; Danilova, T. I.; Antonov, D. Y.; Sergeeva, E. V.; Starikova, Z. A.; Godovikov, I. A.; Il'in, M. M.; Rozenberg, V. I. Isr. J. Chem. 2012, 52, 156-170. 
[65] Morisaki, Y.; Gon, M.; Sasamori, T.; Tokitoh, N.; Chujo, Y. J. Am. Chem. Soc. 2014, 136, 3350-3353.

[66] Sergeeva, E. V.; Shuklov, I. a.; Antonov, D. Y.; Vorontsova, N. V.; Vorontsov, E. V.; Starikova, Z. a.; Il'In, M. M.; Rozenberg, V. I. Tetrahedron: Asymmetry 2010, 21, 1004-1010.

[67] Kubas, A.; Bräse, S.; Fink, K. Chem.-Eur J. 2012, 18, 8377-8385.

[68] Caramori, G. F.; Galembeck, E. J. Phys. Chem. A 2008, 112, 11784-11800.

[69] Zhang, X.-b.; Feng, J.-k.; Ren, A.-m. J. Phys. Chem. A 2007, 111, 13281338.

[70] Masunov, A.; Tretiak, S.; Hong, J. W.; Liu, B.; Bazan, G. C. The Journal of chemical physics 2005, 122, 224505.

[71] Ferrighi, L.; Frediani, L.; Fossgaard, E.; Ruud, K. J. Chem. Phys. 2007, 127.

[72] Bartholomew, G. P.; Rumi, M.; Pond, S. J. K.; Perry, J. W.; Tretiak, S.; Bazan, G. C. J. Am. Chem. Soc. 2004, 126, 11529-11542.

[73] Zhang, X.-b.; Feng, J.-k.; Ren, A.-m.; Sun, C.-C. Can. J. Chem. 2006, 84, 1114-1123.

[74] Caramori, G. F.; Galembeck, S. E. J. Phys. Chem. A 2007, 111, 1705-1712.

[75] Ghiviriga, I.; Martinez, H.; Kuhn, C.; Zhang, L.; Dolbier, W. R. Org. Biomol. Chem. 2012, 10, 882 .

[76] Türker, L.; Atalar, T.; Gümüş, S. Polycycl. Aromat. Comp. 2009, 29, 289314 .

[77] Caramori, G. F.; Galembeck, S. E.; Laali, K. K. J. Org. Chem. 2005, 70, $3242-3250$.

[78] Jiang, B.; Han, L.; Li, Y. L.; Zhao, X. L.; Lei, Y.; Xie, D. Q.; Zhang, J. Z. H. J. Org. Chem. 2012, r7, 1701-1709.

[79] Öström, H. et al. Science 2015, 347, 978-982.

[80] Frisch, M. J. et al. Gaussian09 Revision D.01. Gaussian Inc. Wallingford CT 2009. 
[81] Laury, M. L.; Wilson, A. K. J. Chem. Theory Comput. 2013, 9, 3939-3946.

[82] Miertuš, S.; Scrocco, E.; Tomasi, J. Chem. Phys. 1981, 55, 117-129.

[83] Miertuš, S.; Tomasi, J. Chem. Phys. 1982, 65, 239-245.

[84] Pascual-Ahuir, J. L.; Silla, E.; Tuñón, I. J. Comp. Chem. 1994, 15, 11271138.

[85] Cossi, M.; Barone, V.; Cammi, R.; Tomasi, J. Chem. Phys. Lett. 1996, 255, 327-335.

[86] Barone, V.; Cossi, M.; Tomasi, J. J. Chem. Phys. 1997, 107, 3210-3221.

[87] Cancès, E.; Mennucci, B.; Tomasi, J. J. Chem. Phys. 1997, 107, 3032-3041.

[88] Mennucci, B.; Tomasi, J. J. Chem. Phys. 1997, 106, 5151-5158.

[89] Mennucci, B.; Cancès, E.; Tomasi, J. J. Phys. Chem. B 1997, 101, 1050610517.

[90] Barone, V.; Cossi, M. J. Phys. Chem. A 1998, 102, 1995-2001.

[91] Cossi, M.; Barone, V.; Mennucci, B.; Tomasi, J. Chem. Phys. Lett. 1998, 286, 253-260.

[92] Barone, V.; Cossi, M.; Tomasi, J. J. Comp. Chem. 1998, 19, 404-417.

[93] Cammi, R.; Mennucci, B.; Tomasi, J. J. Phys. Chem. A 1999, 103, 91009108.

[94] Cossi, M.; Barone, V.; Robb, M. A. J. Chem. Phys. 1999, 111, 5295-5302.

[95] Tomasi, J.; Mennucci, B.; Cancès, E. J. Mol. Struct. (Theochem) 1999, 464, 211-226.

[96] Cammi, R.; Mennucci, B.; Tomasi, J. J. Phys. Chem. A 2000, 104, 56315637.

[97] Cossi, M.; Barone, V. J. Chem. Phys. 2000, 112, 2427-2435.

[98] Cossi, M.; Barone, V. J. Chem. Phys. 2001, 115, 4708-4717.

[99] Cossi, M.; Rega, N.; Scalmani, G.; Barone, V. J. Chem. Phys. 2001, 114, $5691-5701$ 
[100] Cossi, M.; Scalmani, G.; Rega, N.; Barone, V. J. Chem. Phys. 2002, 117, 43-54.

[101] Cossi, M.; Rega, N.; Scalmani, G.; Barone, V. J. Comp. Chem. 2003, 24.

[102] Tomasi, J.; Mennucci, B.; Cammi, R. Chem. Rev. 2005, 105, 2999-3093. 